Leonardo Gouvêa e Silva Fortaleza

Non-ferromagnetic metallic foreign body detection by eddy currents

Dissertation presented to the Programa de Pós-Graduação em Metrologia of PUC-Rio as partial fulfillment of the requirements for the degree of Mestre em Metrologia.

Advisor: Prof. Carlos Roberto Hall Barbosa Co-Advisor: Prof. Elisabeth Costa Monteiro Co-Advisor: Prof. Eduardo Costa da Silva

Rio de Janeiro

September 2016 


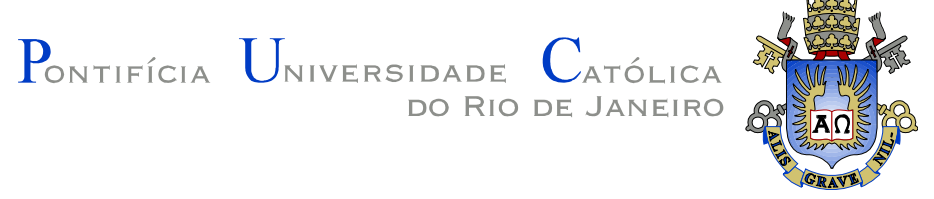

Leonardo Gouvêa e Silva Fortaleza

\section{Non-ferromagnetic metallic foreign body detection by eddy currents}

Dissertation presented as partial fulfillment of the requirements for the degree of Mestre by the Programa de Pós-Graduação em Metrologia of PUC-Rio.

Prof. Carlos Roberto Hall Barbosa

Advisor

Programa de Pós-Graduação em Metrologia - PUC-Rio

Prof. Elisabeth Costa Monteiro

Co-Advisor

Programa de Pós-Graduação em Metrologia - PUC-Rio

Prof. Eduardo Costa da Silva

Co-Advisor

Departamento de Engenharia Elétrica - PUC-Rio

Prof. Márcio Nogueira de Souza

COPPE/UFRJ

Prof. José Franco Machado do Amaral UERJ

Prof. Jorge Luís Machado do Amaral UERJ

Prof. Marcio da Silveira Carvalho Sector Coordinator of Centro Técnico Científico - PUC-Rio

Rio de Janeiro, September $28^{\text {th }}, 2016$ 
All rights reserved.

\section{Leonardo Gouvêa e Silva Fortaleza}

Graduated in Electrical Engineering (majors in Electrical Energy Systems and in Electronics and Computers) by the Pontifical Catholic University of Rio de Janeiro (PUC-Rio), in 2014. Takes part in research conducted in the Laboratory of Biometrology (LaBioMet) and the Laboratory of Research and Development in Electronics (LPDE), both in PUC-Rio. Mainly acts in the field of magnetic transducers, in particular those based on the effect of giant magnetoimpedance. Projects include homogenization of phase characteristics of GMI sensor elements and detection of non-ferromagnetic foreign bodies by means of eddy currents induction.

Bibliographic data

Fortaleza, Leonardo Gouvêa e Silva

Non-ferromagnetic metallic foreign body detection by eddy currents / Leonardo Gouvêa e Silva Fortaleza ; advisers: Carlos Roberto Hall Barbosa, Elisabeth Costa Monteiro, Eduardo Costa da Silva. - 2016.

140 f. : il. color. ; $30 \mathrm{~cm}$

Dissertação (mestrado)-Pontifícia Universidade Católica do Rio de Janeiro, Centro Técnico Científico, Programa de Pós-Graduação em Metrologia, 2016.

Inclui bibliografia

1. Metrologia - Teses. 2. Corpos estranhos metálicos não-ferromagnéticos. 3. Correntes Parasitas. 4. Transdutores Magnéticos. 5. Gradiômetro. 6. GMI. I. Barbosa, Carlos Roberto Hall. II. Monteiro, Elisabeth Costa. III. Silva, Eduardo Costa da. IV. Pontifícia Universidade Católica do Rio de Janeiro. Centro Técnico Científico. Programa de Pós-Graduação em Metrologia. V. Título. 


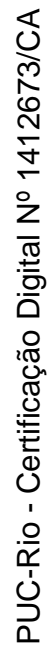

For my parents, Teresa and Luiz Eduardo. 


\section{Acknowledgements}

To my advisers Carlos Roberto Hall Barbosa, Elisabeth Costa Monteiro and Eduardo Costa da Silva for their teachings, their partnership and their guidance.

To the electronics wizard Luiz Antônio Pereira de Gusmão, whose assistance, expertise and wisdom were invaluable.

To CNPq and to PUC-Rio for the grants conceded that were essential for the realization of this work.

To my parents, for all the years of support.

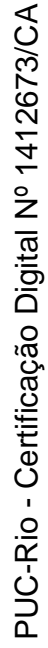

To the professors of the examination comission.

To all the professors for their teachings.

To the staff of the Department for all their assistance.

To my friends and colleagues.

To God, for everything. 


\section{Abstract}

Fortaleza, Leonardo Gouvêa e Silva; Barbosa, Carlos Roberto Hall (Advisor); Monteiro, Elisabeth Costa (Co-Advisor); Costa da Silva, Eduardo (Co-Advisor). Non-ferromagnetic metallic foreign body detection by eddy currents. Rio de Janeiro, 2016. 140p. MSc. Dissertation - Programa de Pós-Graduação em Metrologia, Pontifícia Universidade Católica do Rio de Janeiro.

Lead projectiles (non-ferromagnetic) are common foreign bodies in the medical practice. Conventional means of location use ionizing radiation, pose health risks and lead to procedures that last several hours, typically ending unsuccessfully. Magnetic field maps obtained non-invasively and innocuously with SQUIDs benefit the location of ferromagnetic metallic needles, reducing the time of successful removal from 6 hours to 10 minutes. SQUIDs are currently the most sensitive magnetometers, however require cryogenic temperatures, leading to high cost and low portability which prevent widespread clinical use. The objective is to design a device for locating non-ferromagnetic metallic foreign bodies for surgical removal, respecting project requirements of: high sensitivity, innocuousness, non-invasiveness, low cost, safety, portability, ease of use and room temperature operation. GMR and GMI sensors are considered as more suitable alternatives. Classical electrodynamics theoretical models applied to eddy currents induction serve as framework. Two electronic location systems are developed in gradiometric configuration to remove environmental interference, using commercially available GMR and GMI sensor elements. System performance is obtained from simulation results, demonstrating the capability of detecting the magnetic flux density levels expected under certain projectile radii and distances. The GMI system is more qualified, as its higher sensitivity and improved resolution favors larger measurement ranges, innocuousness, safety and ease of use. The results prove the viability of using GMI sensor elements in this application. The benefits of lower cost, higher portability and safety facilitate the clinical use of more innocuous and effective location techniques for non-ferromagnetic metallic foreign bodies.

\section{Keywords}

Metrology; Non-Ferromagnetic Metallic Foreign Bodies; Eddy Currents; Magnetic Transducers; Gradiometer; GMI; GMR. 


\section{Resumo}

Fortaleza, Leonardo Gouvêa e Silva; Barbosa, Carlos Roberto Hall (Orientador); Monteiro, Elisabeth Costa (Co-orientadora); Costa da Silva, Eduardo (Coorientador). Detecção de corpos estranhos metálicos não-ferromagnéticos por correntes parasitas. Rio de Janeiro, 2016. 140p. Dissertação de Mestrado Programa de Pós-Graduação em Metrologia, Pontifícia Universidade Católica do Rio de Janeiro.

Projéteis de chumbo (não-ferromagnéticos) são corpos estranhos comuns na prática médica. Métodos convencionais de localização utilizam radiações ionizantes, impondo riscos à saúde e procedimentos que duram várias horas e tipicamente terminam malsucedidos. Mapas de campos magnéticos obtidos não-invasivamente e inocuamente com SQUIDs beneficiam a localização de agulhas metálicas ferromagnéticas, reduzindo o tempo de remoção bem-sucedida de 6 horas para 10 minutos. SQUIDs são os magnetômetros mais sensíveis, entretanto requerem temperaturas criogênicas, levando a altos custos e baixa portabilidade que impedem a difusão do uso clínico. O objetivo é desenvolver um dispositivo para localizar corpos estranhos metálicos não-ferromagnéticos visando remoção cirúrgica, respeitando requerimentos de projeto: alta sensibilidade, inocuidade, não-invasividade, baixo custo, segurança, portabilidade, facilidade de uso e operação em temperatura ambiente. Sensores GMR e GMI são considerados alternativas mais adequadas. Modelos teóricos de eletrodinâmica clássica aplicados às correntes parasitas servem como base. Dois sistemas eletrônicos são desenvolvidos em configuração gradiométrica para remover interferência ambiente, usando elementos sensores GMR e GMI disponíveis comercialmente. O desempenho é obtido com resultados de simulações, provando a capacidade de detecção de níveis esperados de densidade de fluxo magnético para certos raios de projéteis e distâncias. O Sistema GMI é mais qualificado, sua mais alta sensibilidade e melhor resolução favorecem maiores faixas de medição, inocuidade, segurança e facilidade de uso. Os resultados demonstram a viabilidade dos elementos sensores GMI nessa aplicação. Os benefícios de baixo custo, maior portabilidade e segurança facilitam a utilização clínica de técnicas de localização para corpos estranhos metálicos não-ferromagnéticos mais inócuas e efetivas.

\section{Palavras-chave}

Metrologia; Corpos Estranhos Metálicos Não-Ferromagnéticos; Correntes Parasitas; Transdutores Magnéticos; Gradiômetro; GMI; GMR. 


\section{Summary}

1 Introduction 21

1.1. Context 21

1.1.1. Surgical removal of foreign bodies 21

1.1.2. Magnetic foreign body detection with SQUID sensors 21

1.1.3. Non-magnetic metallic foreign bodies 22

1.2. Objective 22

1.3. Detection Concept 22

1.4. Metrological reliability of medical electrical equipment 24

1.4.1. International context 24

1.4.2. Brazilian national context 28

1.4.3. Hierarchical Relations of Metrology Organizations 30

1.5. Magnetic Quantities 32

1.6. Dissertation Structure 33

2 Metallic Foreign Body Detection 35

2.1. History 35

2.1.1. Locating steel needles 35

2.1.2. Locating firearm projectiles 39

2.2. High Sensitivity Magnetometers 42

2.2.1. Superconducting Quantum Interference Device (SQUID) 43

2.2.2. Giant Magnetoresistance (GMR) 44

2.2.3. Giant Magnetoimpedance (GMI) 45

$\begin{array}{ll}3 \text { Eddy Currents } & 47\end{array}$

$\begin{array}{ll}\text { 3.1. Theoretical Formulation } & 47\end{array}$

3.1.1. Eddy Currents in Spherical Coordinates 48

3.1.2. Steady State Solution for Eddy Currents in Spherical Coordinates 51

3.1.3. Eddy Currents in Conducting Spheres 52

3.1.4. Eddy Currents Calculations for Magnetometers 55

$\begin{array}{ll}\text { 3.1.5. Gradiometer effects } & 57\end{array}$ 
3.2. Simulated Results and Preliminary Conclusions for Excitation Parameters

4 Systems for Locating Non-Ferromagnetic Metallic Foreign Bodies $\quad 60$

4.1. Developments Based on GMR Sensors 61

4.1.1. Model Used: NVE AA-005-002 61

4.1.2. Primary Magnetic Field Generator Design 64

4.1.3. Primary Magnetic Field Generator Excitation 66

4.1.4. Signal Processing Circuit for Gradiometric Reading 71

4.2. Developments Based on GMI Sensors 83

4.2.1. Model used: Aichi Multi-type Nano-tesla Sensor (MI-CB-1DJ-M-B)83

4.2.2. Primary Magnetic Field Generator Design 87

4.2.3. Signal Processing Circuit for Gradiometric Reading 91

ङ 5 Discussions, Conclusions and Future Works 111

$\begin{array}{ll}\text { 5.1. Discussions } & 111\end{array}$

5.1.1. Major Project Requirements for Biomedical Application 111

$\begin{array}{ll}\text { 5.1.2. Metrological Reliability } & 118\end{array}$

5.2. Conclusions 125

$\begin{array}{ll}\text { 5.3. Future Works } & 126\end{array}$

5.3.1. Optimizing the Location System Design 126

5.3.2. Signal Post-Processing and Location Algorithms 126

$\begin{array}{lr}\text { Bibliography } & 128\end{array}$

Appendix A: Instrumentation Amplifier INA129 132

Appendix B: Precision Full-Wave Rectifiers in the GMI System 134 


\section{List of Figures}

Figure 1 - Simplified diagram of the detection procedure. The solenoid generates a time-varying primary magnetic flux density $B_{o}$ (dashed line in blue) towards the spherical foreign body that induces the also time-varying eddy current loop $I_{e d d y}$ (dotted line in red), in return producing the secondary magnetic flux density $B_{s}$ (dash-dot line in green). The result of a gradiometric (differential) reading by sensor elements S1 and S2 is mostly composed of the secondary magnetic flux density.

Figure 2 - Hierarchical diagram of main organizations involved in the metrological reliability of medical electrical devices in Brazil. The color code is as follows: gold - international metrology organizations, blue - international standardization institutions, black - international scientific organization for public health, green - national institutions.

Figure 3 - Simplified diagrams showing main parameters depth $h$ and angle $\alpha$ of the needle relative to the skin. Also shown are the curves of the magnetic flux density in the $\mathrm{z}$ axis versus the distance in the $\mathrm{x}$ axis for two cases: $\alpha=0^{\circ}$ and $0^{\circ}<\alpha<90^{\circ}$.

Figure 4 - Simulated normal component of the magnetic flux density generated by a $4 \mathrm{~cm}$ needle with $\alpha=30^{\circ}$ and $h=10 \mathrm{~cm}$, showing the extrema parameters $M$ and $m$, respectively the maximum and minimum magnitudes of the magnetic flux density, as well as the displacement $\Delta$ from the needle center and the position $D$ between the extrema. [1]

Figure $5-\mathrm{X}$-ray film of the sixth patient indicating the steel needle location obtained by means of the procedure using SQUID magnetic mapping. The marker is above a very small steel needle fragment that, previously to the magnetic field measurement, was considered to be an artifact in the x-ray film [1]. 
Figure 6 - Simulated normalized magnetic flux density generated by a $4.5 \mathrm{~cm}$ needle with $\alpha=40^{\circ}$ and $h=12 \mathrm{~cm}$, showing the width parameters $W$ and $w$, as well as the displacement $\Delta$ and the distance $D$ between extrema [2].

Figure 7 - Simplified diagram of two experimental setups using a SQUID dewar: using a solenoid on the left and using a Helmholtz coil on the right. [3]

Figure 8 - Simulation results of the technique. On the left, normalized $z$ axis component of the secondary magnetic flux density $\mathrm{Bz}$ measured over the $x$ axis at the depths of 5,10 and $15 \mathrm{~cm}$. On the right, phase of the secondary magnetic flux density (in relation to primary magnetic flux density) versus the radius of the spherical foreign body, measured at frequencies of 50, 100, 200, 500 and $1000 \mathrm{~Hz} .[3]$.

Figure 9 - Minimum detectable radius using a solenoid and a SQUID based first order gradiometer, considering a minimum detectable magnetic field of $10 \mathrm{pT}$ for each depth, for three values of the excitation frequency $(50,100 \mathrm{~Hz}$, and $1 \mathrm{kHz})[3]$

Figure 10 - Simulated results for secondary magnetic fields due to eddy currents at a spherical foreign body made of lead with radii between $1 \mathrm{~mm}$ and $5 \mathrm{~mm}$ at a depth $h=10 \mathrm{~cm}$, presented in relative peak magnetic flux density (BsmaxB0) amplitude and phase in function of the frequency fo. The respective radii for each curve are shown at right

Figure 11 - Block diagram of a generic foreign body detection system circuit. 60

Figure 12 - Percentage of the peak magnetic flux density emitted by the solenoid with the distance, showing a high attenuation with the distance. The cursor indicates the edge of the solenoid at $D=0 \mathrm{~cm}$, where one GMR sensor is to be positioned. The other would be symmetrically located at $\mathrm{D}=-5 \mathrm{~cm}$. 
Figure 13 - Schematic for the primary magnetic field generator excitation circuit, responsible for reducing the supply voltage even for high excitation currents.

Figure 14 - Simulation results of voltage over solenoid $\left(\mathrm{V}_{\text {sol }}\right)$, voltage over capacitor ( $\left.\mathrm{V}_{\text {cap }}\right)$ and current over the solenoid (IsoL) under $V_{A C}$ $=100 \mathrm{mV}$ and $V_{D C}=400 \mathrm{mV}$

Figure 15 - Experimental values of voltages over the solenoid $\left(\mathrm{V}_{\text {sol }}\right)$ and over the capacitor $\left(\mathrm{V}_{\text {cap }}\right)$.

Figure 16 - Experimental values of MOSFET voltages: (a) source voltage $V_{S}$ (used to obtain the $I_{D}$ current values) and (b) drain voltage $\mathrm{V}_{\mathrm{D}}$.

Figure 17 - Simplified schematic for the signal processing circuit including the GMR sensor packages, showing their internal bridge composition.

Figure 18 - Curves for the peak secondary magnetic flux density (Bsmax) (in $\mathrm{T}$ ) varying with the diameter of the foreign body (in $\mathrm{mm}$ ), for a given distance (in $\mathrm{cm}$ ). Each curve's number at right represents the distance in $\mathrm{cm}$ between the projectile and the detector in that case. The red line represents the estimated resolution of the GMR detection system at $2 \mu \mathrm{T}$. .76

Figure 19 - Minimum detectable diameter of the non-magnetic metallic foreign body varying with the distance from the GMR location system.

Figure 20 - Simulated results for output voltage amplitude (V) versus secondary magnetic flux density $(\mu \mathrm{T})$ on the GMR signal processing circuit for gradiometric reading. Samples between 100 $\mu \mathrm{T}$ to $1000 \mu \mathrm{T}$ were obtained in $50 \mu \mathrm{T}$ steps. .78

Figure 21 - Region considered linear for the output voltage amplitude (V) versus secondary magnetic flux density $(\mu \mathrm{T})$ on the GMR signal processing circuit for gradiometric reading. This ranges from 0 to approximately $400 \mu \mathrm{T}$. 
Figure 22 - Output voltage amplitude (V) of the GMR system for secondary magnetic flux densities $(\mu \mathrm{T})$ between 0 to $100 \mu \mathrm{T}$ in 5 $\mu \mathrm{T}$ steps (close to the estimated resolution of the sensor at $2 \mu \mathrm{T}$ ).

Figure 23 - Logarithmic plot comparison of output amplitudes (V) between an individual GMR sensor and the complete detection system versus the secondary magnetic flux density (mT). The GMR curve is valid for the operating point of $40 \mathrm{Oe}=4 \mathrm{mT}$ DC primary flux density. The system output has the amplitude offset of 17.43 $\mathrm{mV}$ subtracted.

Figure 24 - Gain introduced by the signal processing circuit over the GMR sensor in $\mathrm{V} / \mathrm{V}$ calculated for each simulated point for secondary magnetic fields ranging from $5 \mu \mathrm{T}$ to $400 \mu \mathrm{T}$.

Figure 25 - Simplified diagram with dimensions of the GMI sensor prototype Aichi MI-CB-1DJ-M-B.

Figure 26 - Sensitivity curve for the GMI sensor Aichi MI-CB-1DJ-M-

B obtained from characterization studies, given in voltage output versus magnetic flux density at the center of the Helmholtz coil for excitation frequency $f_{o}=8 \mathrm{kHz}$. Also displayed is the linear approximation for the curve.

Figure 27-Region considered linear for the sensitivity curve of the GMI sensor Aichi MI-CB-1DJ-M-B, again for $f_{o}=8 \mathrm{kHz}$.

Figure 28 - Matlab model of the solenoid responsible for generating the primary magnetic field when using the GMI sensors. Spiral characteristics are $10 \mathrm{~mm}$ radius, $70 \mathrm{~mm}$ height and 36 turns evenly spaced.

Figure 29 - Colormap of the magnetic flux density in the $\mathrm{z}$ axis direction within the solenoid on the $\mathrm{x}=0.0 \mathrm{~m}$ plane.

Figure 30 - Magnetic flux density on the solenoid axis for varying heights using a $2 \mathrm{~mA}$ excitation current.

Figure 31 - Average magnetic flux density at the z-axis direction over the superior GMI sensor element, centered at approximately $\mathrm{z}=$ $6.75 \mathrm{~mm}$. 
Figure 32 - Average magnetic flux density at the z-axis direction over the inferior GMI sensor element, centered at approximately $z=-$ $63.25 \mathrm{~mm}$.

Figure 33 - Block diagram of the implemented circuit. "BP Filter" stands for bandpass filter.

Figure 34 - VCVS bandpass filter topology (second order). [38]

Figure 35 -Selected Fourth Order VCVS Bandpass Filter.

Figure 36 - Bandpass filter simulated frequency response in magnitude (linear scale). 95

Figure 37 - Bandpass filter simulated frequency response in magnitude (dB scale) and phase.

Figure 38 - Bandpass filter simulated frequency response in magnitude ( $\mathrm{dB}$ scale) and phase, focusing on the passband. The cutoff frequencies are marked with the cursors. 96

Figure 39 - Results of prototype filter characterization in magnitude frequency response (linear scale) in blue. The orange line is the simulated response.

Figure 40 - Results of prototype filter characterization in magnitude frequency response ( $\mathrm{dB}$ scale) in blue. The orange line is the simulated response.

Figure 41 - Schematic for the detection system prototype.

Figure 42 - Curves of peak secondary magnetic flux density magnitude (Bmax) versus the diameter of the foreign body for different heights. The blue number on the right indicates the height value in $\mathrm{cm}$ of the respective curve. The reference line represents the estimated resolution of $25 \mathrm{pT}$.

Figure 43 - Comparison of GMI and GMR performance regarding minimum detectable diameter over height using the estimated resolutions for each detection system $(2 \mu \mathrm{T}$ for the GMR system and $25 \mathrm{pT}$ for the GMI system).

Figure 44 - GMI system output amplitude (V) for gain of $1.988 \mathrm{~V} / \mathrm{V}$ for secondary magnetic flux densities varying from 0 to $1000 \mathrm{nT}$. 
Figure 45 - GMI system output amplitude (V) for gain of $10.88 \mathrm{~V} / \mathrm{V}$ for secondary magnetic flux densities varying from 0 to $1000 \mathrm{nT}$.

Figure 46 - GMI system output amplitude (V) for gain of $99.8 \mathrm{~V} / \mathrm{V}$ for secondary magnetic flux densities varying from 0 to $100 \mathrm{nT}$.

Figure 47 - GMI system output amplitude (V) for gain of $989 \mathrm{~V} / \mathrm{V}$ for secondary magnetic flux densities varying from 0 to $10 \mathrm{nT}$.

Figure 48 - Logarithmic plot for the GMI system output amplitude (V) versus secondary magnetic flux density including the four simulated gains, the experimental GMI characterization and the GMI theoretical linear range output.

Figure 49 - Logarithmic plot for the gain introduced by the GMI detection system $(\mathrm{V} / \mathrm{V})$ versus secondary magnetic flux density for the four simulated gains. The amplitude offsets were removed.

Figure 50 - GMI system output voltage under a secondary magnetic field of $25 \mathrm{pT}$ for $\phi_{\text {sec }}$ phases between the primary and secondary magnetic fields ranging from $-90^{\circ}$ to $-180^{\circ}$ in $-15^{\circ}$ steps. The instrumentation amplifier gain is set at $989 \mathrm{~V} / \mathrm{V}$.

Figure 51 - GMI system normalized response to the secondary magnetic field phase for different gains, using the output amplitude at a phase of $-90^{\circ}$ as reference. All values consider the $25 \mathrm{pT}$ resolution level.

Figure 52 - Logarithmic plot comparison between sensor elements and location systems in output amplitude over secondary magnetic flux density.

Figure 53 - Comparison of GMI, GMR and SQUID performance regarding minimum detectable diameter over height using the estimated resolutions for each detection system.

Figure 54 - Minimum detectable diameter over height for the proposed GMI, GMR and SQUID systems (solid lines) and adapted versions using the ICNIRP magnetic flux density reference levels, satisfying occupational and general public exposure at the corresponding frequencies (dashed lines). 
Figure 55 - Precision full-wave rectifier design intended to prevent phase mismatch between the instrumentation amplifier inputs. It requires that the resistors $\mathrm{R} 2, \mathrm{R} 3, \mathrm{R} 4$ and $\mathrm{R} 5$ have the same nominal value and very low tolerances while the input resistance $\mathrm{R} 1=$ Rin can be used for small gain adjustment.

Figure 56 - Amplitudes of the instrumentation amplifier outputs (with 99.8 V/V gain) without any rectifier at various $\phi_{\text {pri }}$ phase differences between the primary magnetic field signals, specifically: $0^{\circ}, 0.01^{\circ} 0.1^{\circ}, 1^{\circ}$ and $10^{\circ}$. The y-axis is in logarithmic scale.

Figure 57 - Instrumentation amplifier outputs (with $99.8 \mathrm{~V} / \mathrm{V}$ gain) when using the precision rectifiers at various $\phi$ pri phase differences between the primary magnetic field signals, specifically: $0^{\circ}, 0.01^{\circ}$ (@2),0.1(@3),1(@4) and 10(@5).....

Figure 58 - Signal of interest under a secondary magnetic field of $10 \mathrm{nT}$ for $\phi_{\text {sec }}$ phases between the primary and secondary magnetic fields ranging from $-90^{\circ}$ to $-180^{\circ}$ in $-15^{\circ}$ steps. These signals were obtained in the simulations by mathematically subtracting the GMI sensor outputs.

Figure 59 - Instrumentation amplifier outputs when using $99.8 \mathrm{~V} / \mathrm{V}$ gain and the precision rectifiers for a secondary magnetic flux density of $10 \mathrm{nT}$ with $\phi_{\mathrm{sec}}$ phases between the primary and secondary magnetic fields ranging from $-90^{\circ}$ to $-180^{\circ}$ in $-15^{\circ}$ steps. 


\section{List of Tables}

Table 1- Magnetic quantities and its SI units................................................32

Table 2 - Units of magnetic quantities in the CGS system and their

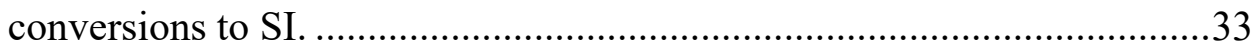

Table 3- Main characteristics for GMR sensor NVE AA-005-02 ...................64

Table 4 - Main characteristics for GMI sensor Aichi MI-CB-1DJ-M-B..........85

Table 5 - Maximum signal of interest for several differential gains applied in the GMI detection system...............................................100

Table 6 - Optimal measurement ranges for each simulated differential

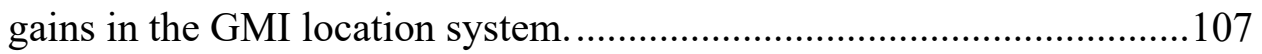

Table 7 - Comparison between theoretical and effective output gain $(\mathrm{V} / \mathrm{V})$ considering the simulation results.

Table 8 - Innocuousness analysis of the location systems using ICNIRP reference levels.

Table 9 - Analysis of the major biomedical device requirements for the considered magnetometers and their respective location systems.

Table 10 - Analysis of the major metrological reliability requirements for the considered magnetometers and their respective location systems. 


\section{List of Symbols}

$a$ - Radius

$A$ - Magnetic vector potential

$B$ - Magnetic flux density

$C M R R$ - Common-mode rejection ratio

E - Electric field

$G_{\text {dif }}$ - Differential gain

$G_{c m}$ - Common-mode gain

$H$ - Magnetic field strength

J - Current density

$l_{s}$ - Base-line

Sens - Sensitivity

$S N R$ - Signal-to-noise ratio

$\mu$ - Permeability

$\mu_{o}$ - Permeability constant or permeability of free space or magnetic constant

$\mu_{r}-$ Relative permeability

$\rho$ - Resistivity

$\sigma-$ Electrical conductivity

$\delta$ - Eddy current skin-depth

$\phi-$ Magnetic flux

$\phi_{E}-$ Electric potential

$\phi$ pri -Phase between the two signals pertaining to the primary magnetic field

$\phi$ sec - Phase between the secondary and the primary magnetic fields 


\section{List of Abbreviations}

ABNT - Associação Brasileira de Normas Técnicas or Brazilian National Standards Organization

AC - Alternating Current

ANVISA - Agência Nacional de Vigilância Sanitária or (Brazilian) National Health Surveillance Agency

BIPM - Bureau International des Poids et Mesures or International Bureau of Weights and Measures

CCEM - Comité Consultatif d'Électricité et Magnétisme or Consultative Committee on Electricity and Magnetism

CGPM - Conférence Générale des Poids et Mesures or General Conference on Weights and Measures

CIPM - Comité International des Poids et Mesures or International Committee on Weights and Measures

$\mathrm{CMI}$ - Czech Metrology Institute

DC - Direct Current

GMI - Giant Magnetoimpedance

GMR - Giant Magnetoresistance

GUM - Glowny Urzad Miar or (Polish) Central Office of Measures

ICNIRP - International Commission on Non-Ionizing Radiation Protection

IEC - International Electrotechnical Commission

INMETRO - Instituto Nacional de Metrologia, Qualidade e Tecnologia or (Brazilian) National Institute of Metrology, Quality and Technology ISO - International Organization for Standardization

LAMCE - Laboratório de Magnetismo e Campo Elétrico e Magnético or Laboratory of Magnetism and Electric and Magnetic Field

MEES - Medical Electrical Equipment Safety

$\mathrm{NMI}$ - (Australia) National Measurement Institute

NPL - (UK) National Physics Laboratory

OIML - Organisation Internationale de Métrologie Légale or International Organization of Legal Metrology 
RDC - Rede Brasileira de Calibração or Brazilian Calibration Network

SQUID - Superconducting Quantum Interference Device

VIM - Vocabulaire International de Métrologie or International Vocabulary

of Metrology

VSL - Van Swinden Laboratory (Netherlands)

WHO - World Health Organization 


\section{Introduction}

\section{1.}

\section{Context}

\subsection{1.}

\section{Surgical removal of foreign bodies}

There is a high incidence of foreign bodies in patients, often requiring surgical removal [1-3]. Locating these objects is usually difficult. Currently, the only widely available means of locating foreign objects are radiography, computed tomography and radioscopy procedures. These methods are often ineffective, leading to procedures that last several hours and typically end unsuccessfully, especially when dealing with small objects [1]. Additionally, these imaging techniques use ionizing radiation, therefore posing risks to patients and to the medical staff.

\subsection{2.}

Magnetic foreign body detection with SQUID sensors

Studies conducted in the Laboratory of Biometrology (LaBioMet) at Pontifical Catholic University of Rio de Janeiro (PUC-Rio) demonstrated that the use of magnetic field maps can greatly benefit the location of magnetic foreign bodies, reducing the time spent to successfully remove magnetic metallic needles from 6 hours (without success) to around 10 minutes [1]. In addition, the detection of objects by high sensitivity magnetic transducers is non-invasive and innocuous, providing simplicity of operation and diminishing risks to both staff and patients. In these procedures, a Superconducting Quantum Interference Device (SQUID) was used as the sensor element.

SQUID sensors are currently the most sensitive magnetometers, presenting extremely low noise levels. However, their operation requires cryogenic temperatures, typically supplied by liquid Helium cooling. This characteristic leads to high cost and low portability, preventing the widespread use of this equipment, especially in the clinical environment [1-4]. 


\subsection{3.}

\section{Non-magnetic metallic foreign bodies}

Firearm projectiles pose an even greater detection challenge due to their lack of ferromagnetism (lead, for instance, is diamagnetic). A theoretical method was proposed to induce a secondary magnetic field associated to metallic projectiles by the generation of eddy currents, caused by a sinusoidal primary magnetic field [3,5]. The secondary magnetic field induced can be measured by high sensitivity magnetic sensors.

\section{2. Objective}

The main objective of this work is to design and develop a device capable of locating non-ferromagnetic metallic foreign bodies for surgical removal, respecting some major project requirements: high sensitivity, innocuousness, noninvasiveness, low cost for clinical application, safety, portability, ease of use and capacity to operate at room temperature [4,6]. SQUID magnetometers fail to comply with some of these requirements, therefore alternatives were considered, in particular giant magnetoresistance (GMR) and giant magnetoimpedance (GMI) sensors.

\section{3. \\ Detection Concept}

The basic detection concept consists of using a time-varying primary magnetic flux density generator to induce eddy current loops in the foreign body, which consequently produce a secondary magnetic flux density to be measured by a gradiometric (differential) reading system, composed by two sensor elements. Fig. 1 illustrates the detection concept in a simplified manner, considering a case with the solenoid axis aligned with the center of the spherical foreign body. This axis is defined as the $\mathrm{z}$ axis, over which the distance or depth values are measured. 


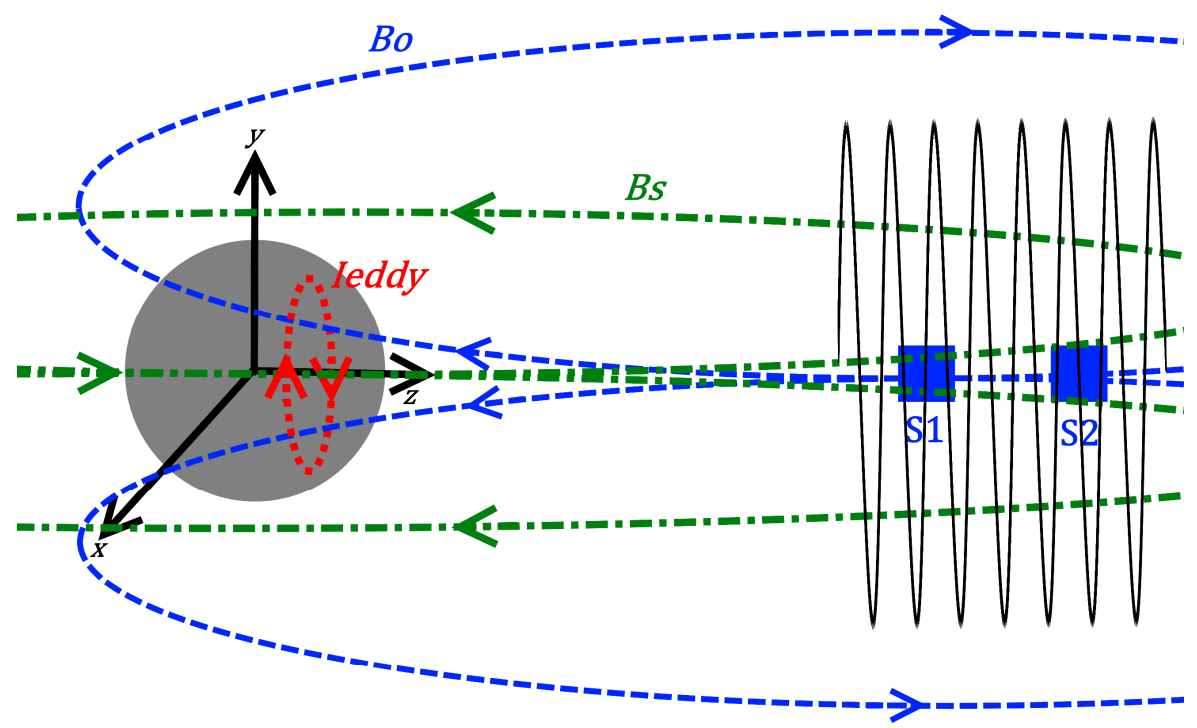

Figure 1 - Simplified diagram of the detection procedure. The solenoid generates a time-varying primary magnetic flux density $B_{o}$ (dashed line in blue) towards the spherical foreign body that induces the also time-varying eddy current loop $I_{\text {eddy }}$ (dotted line in red), in return producing the secondary magnetic flux density $B_{s}$ (dash-dot line in green). The result of a gradiometric (differential) reading by sensor elements $\mathrm{S1}$ and $\mathrm{S} 2$ is mostly composed of the secondary magnetic flux density.

The primary magnetic field generator in this case is a solenoid excited by an alternating current (AC). The variation of the primary magnetic flux density $B_{o}$ induces eddy current loops in the foreign body, exemplified by $I_{e d d y}$ in Fig. 1. These currents are also time-varying, inducing an AC secondary magnetic flux density $B_{s}$ whose intensity is several orders of magnitude smaller than $B_{o}$. This accentuates even further taking into account the high reduction of the magnetic flux density over distance.

Two sensor elements S1 and S2 are placed symmetrically on the solenoid, ideally receiving equal values of $B_{o}$ and other environmental interferences, such as the Earth's magnetic field. The closer proximity of S1 to the foreign body leads to the measurement of considerably higher magnitude values of $B_{s}$ compared to S2. Therefore, the gradiometric or differential reading using S1 and S2 is mostly composed of the secondary magnetic flux density $B_{s}$, which is the signal of interest. 


\section{4. \\ Metrological reliability of medical electrical equipment}

Metrology, defined as the science of measurement [7], can be divided in three major aspects: Scientific Metrology, Industrial Metrology and Legal Metrology [8]. Scientific Metrology studies natural phenomena in order to organize, establish and maintain standards for measurement units. Industrial Metrology ensures the proper use of measurements in industry. Legal Metrology deals with regulations and guidelines that focus on the proper use of measurements in the commercial trade, health, safety and environment sectors.

The following items are short descriptions of the most relevant organizations responsible for metrological reliability in the international and national spheres (in Brazil).

\subsection{1. International context}

\subsubsection{1. BIPM - International Bureau of Weights and Measures}

BIPM (Bureau International des Poids et Mesures) is an intergovernmental organization established in 1875 at the Metre Convention in Paris. It is headquartered in Sèvres, France. Its main goal is the establishment of a single coherent and universally accepted measurement system traceable to the International System of Units (SI) [9].

The BIPM runs under the authority of the General Conference on Weights and Measures (CGPM - Conférence Générale des Poids et Mesures), a meeting held by delegates of all member states every four years. During these meetings occurs an analysis of reports and proposals made by the International Committee on Weights and Measures (CIPM - Comité International des Poids et Mesures), an advisory board consisting of prominent metrologists. This leads to the institution of several international scientific resolutions by the CGPM.

Under the CIPM there are several consultative committees that support their work by focusing on a research field. The most relevant committee for medical 
electrical equipment dealing with magnetic quantities, scope of this present work, is the Consultative Committee on Electricity and Magnetism (CCEM - Comité Consultatif d'Électricité et Magnétisme), established in 1997.

\subsubsection{2.}

\section{OIML - International Organization of Legal Metrology}

OIML (Organisation Internationale de Métrologie Légale) is an intergovernmental institution established in 1955 to promote global harmonization of legal metrology procedures, simplifying international trade [10]. Its headquarters is in Paris, France. It produces international recommendations to member states that may be used in domestic laws. The work produced by the OIML seeks to achieve cooperation with standards set by IEC (International Electrotechnical Commission) and ISO (International Organization for Standardization).

There are several Technical Committees working for OIML, each one dedicated to a specific field. The most relevant Committee to medical electrical equipment is TC 18 - Medical measuring instruments, in particular its Subcommittee TC 18/SC 4 - Bio-electrical instruments. Considering the objectives of the present work, another important committee is TC 12 - Instruments for measuring electrical quantities.

\subsubsection{3.}

IEC - International Electrotechnical Commission

IEC is a non-governmental international standards organization for electrical, electronic and related technologies, including medical electrical equipment [11]. It was founded in 1906 and its current headquarters are located in Geneva, Switzerland.

It also includes several Technical Committees responsible for researching and proposing standards in particular fields. The most relevant to the device herein developed are TC 62 - Electrical equipment in medical practice, TC 85 - Measuring equipment for electrical and electromagnetic quantities and TC 108 - Methods for the assessment of electric, magnetic and electromagnetic fields associated with human exposure.

The IEC 60601 series of standards deals with medical electrical equipment. Considering the present work, the most relevant standards are: 
- IEC 60601-1:2005+AMD1:2012: Medical electrical equipment Part 1: General requirements for basic safety and essential performance

- IEC 60601-1-2:2014: Medical electrical equipment - Part 1-2: General requirements for basic safety and essential performance Collateral Standard: Electromagnetic disturbances - Requirements and tests

- IEC 60601-1-6:2010+AMD1:2013: Medical electrical equipment Part 1-6: General requirements for basic safety and essential performance -Collateral standard: Usability

- IEC 60601-1-9:2007+AMD1:2013: Medical electrical equipment Part 1-9: General requirements for basic safety and essential performance - Collateral Standard: Requirements for environmentally conscious design

- IEC 60601-1-12:2014: Medical electrical equipment - Part 1-12: General requirements for basic safety and essential performance Collateral Standard: Requirements for medical electrical equipment and medical electrical systems intended for use in the emergency medical services environment

In addition, the IEC $\mathbf{6 1 7 8 6}$ series deals with the measurement of low-frequency magnetic and electric fields with regard to exposure of human beings:

- IEC 61786:1998: Measurement of low-frequency magnetic and electric fields with regard to exposure of human beings - Special requirements for instruments and guidance for measurements

- IEC 61786-1:2013: Measurement of DC magnetic, AC magnetic and AC electric fields from $1 \mathrm{~Hz}$ to $100 \mathrm{kHz}$ with regard to exposure of human beings - Part 1: Requirements for measuring instruments

- IEC 61786-2:2014: Measurement of DC magnetic, AC magnetic and $\mathrm{AC}$ electric fields from $1 \mathrm{~Hz}$ to $100 \mathrm{kHz}$ with regard to exposure of human beings - Part 2: Basic standard for measurements 


\subsubsection{4.}

ISO - International Organization for Standardization

ISO is a non-governmental international organization founded in 1947 with headquarters in Geneva, Switzerland [12]. Its membership is mostly composed by the most representative national standard body of each country. ISO sets standards in a wide variety of fields and has also formed joint committees with the International Electrotechnical Commission (IEC) in several areas, such as the quality evaluation of medical devices. The relevant Technical Committees are:

- ISO/TC 194: Biological and clinical evaluation of medical devices

- ISO/TC 210: Quality management and corresponding general aspects for medical devices

○ ISO/TC 210/JWG 01: "Joint ISO/TC 210-IEC/SC 62A WG; Application of risk management to medical devices"

○ ISO/TC 210/JWG 02: “Joint ISO/TC 210-IEC/SC 62A WG; Medical device software"

○ ISO/TC 210/JWG 03: "Joint ISO/TC 210-IEC/SC 62A WG; Medical device usability"

○ ISO/TC 210/WG 01: "Application of quality systems to medical devices"

○ ISO/TC 210/WG 02: "General aspects stemming from the application of quality principles to medical devices"

Currently, the most relevant standard for the proposed device is:

- ISO 14971:2007: Medical devices-Application of risk management to medical devices

\subsubsection{5.}

\section{WHO - World Health Organization}

WHO is a specialized agency of the United Nations (UN) dedicated to international public health, established in 1947 and headquartered in Geneva, Switzerland [13]. It acts in several roles, including the monitoring of the health situation worldwide, assessing health trends and setting norms and standards.

In the area of medical devices, it refers to ISO and IEC, while regarding electromagnetic fields and its effects on human health it refers to guidelines 
published by ICNIRP (International Commission on Non-Ionizing Radiation Protection).

\subsubsection{6.}

\section{ICNIRP - International Commission on Non-Ionizing Radiation Protection}

ICNIRP is an independent non-profit and non-governmental scientific organization based in Munich, Germany, and founded in 1992 to continue and complement the work done by the International Radiation Protection Association (IRPA) [14]. It performs scientific research and publishes guidelines, reviews and statements regarding the adverse effects of non-ionizing radiation (static electric fields, static magnetic fields and electromagnetic fields) in human health and the environment. Most of its publications are freely available to the public. The most relevant material for the developed device is:

- ICNIRP Guidelines For Limiting Exposure To Time-Varying Electric And Magnetic Fields (1 Hz-100 kHz) (last updated in 2010)

- ICNIRP Guidelines For Limiting Exposure To Time-Varying Electric, Magnetic and Eletromagnetic Fields (up to $300 \mathrm{GHz}$ ) (last updated in 1998, with revision underway)

- ICNIRP Guidelines On Limits Of Exposure To Static Magnetic Fields (last updated in 2009)

\subsection{2.}

\section{Brazilian national context}

\subsubsection{1.}

\section{INMETRO - National Institute of Metrology, Quality and Technology}

INMETRO (Instituto Nacional de Metrologia, Qualidade e Tecnologia) is a Brazilian federal autarchy founded in 1973, dedicated to metrology in all its sectors (scientific, legal and industrial) [15]. It is associated to the Ministry of Development, Industry and Foreign Commerce (MDIC). Its mission statement is "to support Brazilian enterprises to increase their productivity and the quality of goods and services" as well as to provide confidence to the Brazilian society in measurements and goods, through metrology and conformity evaluation, promoting 
the harmonization of consumer relation, innovation and competitiveness in the country.

INMETRO accredits national certification bodies in Brazil. The traceability of electromagnetic fields measurement is ensured by the Laboratory of Magnetism and Electric and Magnetic Field (LAMCE - Laboratório de Magnetismo e Campo Elétrico e Magnético). It maintains the national measurement standards for these quantities. These are used in calibration services performed by accredited laboratories of the Brazilian Calibration Network (RDC - Rede Brasileira de Calibração) and electrical energy enterprises. Its electric field measurement standard is primary, while its magnetic measurement standard is traceable to the National Measurement Institute of Australia (NMI).

Presently, LAMCE provides traceability to magnetic field flux densities in the range of $1 \mu \mathrm{T}$ to $700 \mu \mathrm{T}$ at the frequencies of $50 \mathrm{~Hz}$ or $60 \mathrm{~Hz}$ [16]. Their current goal is to be capable of calibrating devices for DC fields between $2 \mu \mathrm{T}$ and $2 \mathrm{~T}$ and, subsequently, for $\mathrm{AC}$ fields between $1 \mathrm{nT}$ and $10 \mathrm{mT}$ at frequencies in the range of $10 \mathrm{~Hz}$ to $10 \mathrm{kHz}[17]$.

\subsubsection{2.}

\section{ANVISA - National Health Surveillance Agency}

ANVISA (Agência Nacional de Vigilância Sanitária) is the regulatory body dealing with public health in Brazil, created by Law $n^{\circ} 9.728$ of January 26, 1999 [18]. It is an autarchy under a special regime, which means it has administrative independence and financial autonomy from the government even though it is a public organization related to the Ministry of Health.

In 1999, ANVISA published Resolution $\mathrm{n}^{\circ} 444$, instituting as compulsory the use of the Brazilian technical standards adapted from the IEC 60601 series such as the NBR IEC 60601-1: Equipamento Eletromédico. Parte 1 - Prescrições Gerais para Segurança and particular standards with specific requirements from the IEC 60601-2 series. This resolution was superseded by Resolution RDC n 32 of May 2007, altered in June 2007 and again replaced by the current Resolution RDC n ${ }^{\circ} 27$, of 21 June 2011, that provides the procedures for the mandatory certification of equipment under the Health Surveillance System.

Under Article $2^{\text {nd }}$, RDC 27/2011 states that equipment should comply with Resolution RDC ANVISA 56/2001 which establishes the essential safety and 
effectiveness requirements for healthcare products, by means of certification of conformity under the Brazilian Compliance Assessment System (SBAC - Sistema Brasileiro de Avaliação da Conformidade).

Under the Paragraph 1 of its Article $2^{\text {nd }}$, the RDC 27/2011 indicates the ANVISA's Normative Instruction n. 3, of 21 June 2011, to assess the requirements for conformity assessment, specifying standards that shall be used for the conformity evaluation of medical equipment.

The Paragraph 2 of Article $2^{\text {nd }}$ of RDC 27/2011 establishes that the equipment under the regime of Health Surveillance includes any equipment and its parts and accessories with medical, dental, laboratory or physiotherapy purposes, used directly or indirectly for diagnosis, treatment, rehabilitation and monitoring of human beings, as well as equipment with aesthetical and beautifying purpose.

\subsubsection{3.}

\section{ABNT - Brazilian National Standards Organization}

ABNT (Associação Brasileira de Normas Técnicas) is a private non-profit national standards organization founded in 1940 [19]. Its role is supported by the federal government in several legal instruments. It is one of the founding members of the International Organization for Standardization (ISO). It is responsible for publishing the Brazilian Standards (ABNT NBR), many of which are translated and adapted versions of ISO and IEC standards.

Their work is divided in technical committees. ABNT/CB-026 is the DentalMedical-Hospital Brazilian Committee (Comitê Brasileiro Odonto-MédicoHospitalar), dealing with products associated with health, except those dealing with ionizing radiations. They were responsible by the standard ABNT NBR ISO 14971:2009: Produtos para a saúde - Aplicação de gerenciamento de risco a produtos para a saúde (Medical devices-Application of risk management to medical devices). ABNT/CB-03 is the Electricity Brazilian Committee (Comitê Brasileiro de Eletricidade), dealing with all kinds of electrical, electronic and related fields.

\subsection{3.}

\section{Hierarchical Relations of Metrology Organizations}

Fig. 2 is an attempt to display the hierarchical relations of the major international organizations, as well as the associated national organizations in 
Brazil, that contributes to metrological reliability of medical electrical equipment intending for measuring magnetic fields.

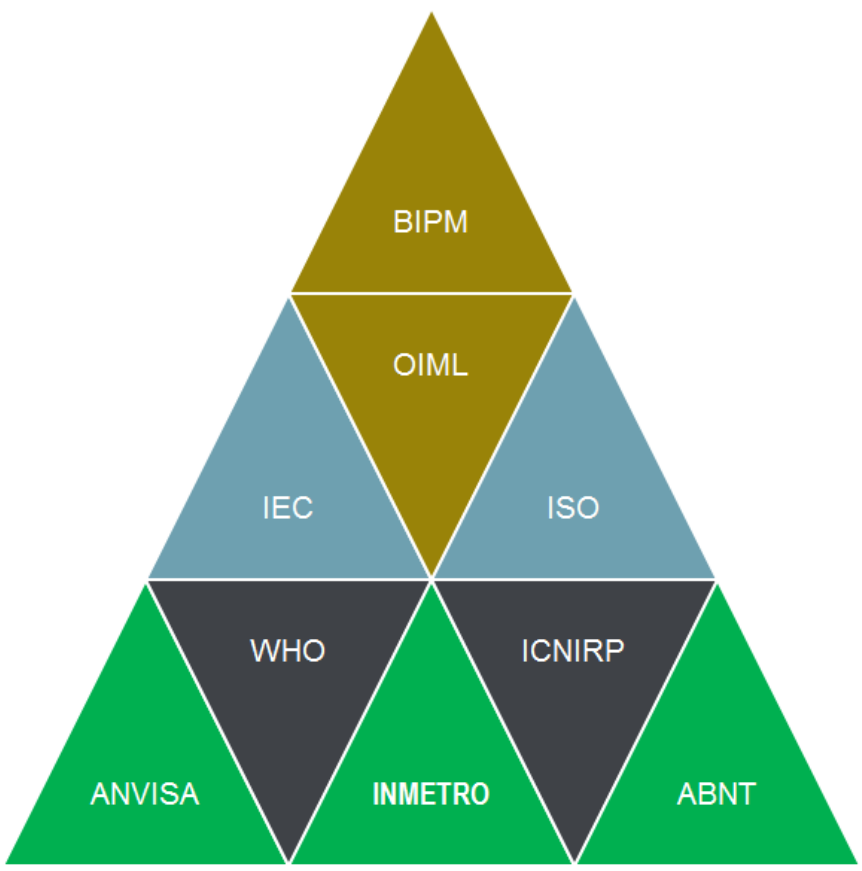

Figure 2 - Hierarchical diagram of main organizations involved in the metrological reliability of medical electrical devices in Brazil. The color code is as follows: gold - international metrology organizations, blue - international standardization institutions, black - international scientific organization for public health, green - national institutions.

The gold color represents the highest level of the hierarchical diagram (Fig. 2), the intergovernmental metrological organizations. BIPM defines and maintains measurement standards and the reference methods and OIML proposes legal support for the harmonization of metrology-related laws and regulations worldwide. The blue color represents private international standards organizations. The black color represents international organizations dedicated to human health, in close relation to the scope of this work. These latter monitor health and safety, introducing guidelines to prevent hazards. The green color represents national organizations in Brazil, responsible by local standards, regulations, conformity assessment and measurement infrastructure regarding metrology and health. 


\section{5 .}

\section{Magnetic Quantities}

The commonly used terminology of some magnetic quantities and its units is often ambiguous, since the use of "magnetic field" for both the $B$ and the $H$ quantities is commonplace. The $B$ quantity is present whenever an electric current exists while the $H$ quantity also considers the magnetization effect present in most materials. The $H$ quantity is more traditionally defined as the "magnetic field" or "magnetic field intensity" or "magnetic field strength", while the $B$ quantity can be called "magnetic flux density" or "magnetic induction". In SI units, both quantities are related by

$$
H=\frac{B}{\mu_{0} \cdot \mu_{r}}-M,
$$

where $\mu_{0}$ is the permeability of free space $\left(4 \pi \cdot 10^{-7} \mathrm{H} / \mathrm{m}\right), \mu_{r}$ is the relative permeability (in vacuum or in a non-ferromagnetic material, it is approximately equal to 1) and $M$ is the magnetization field vector, which only exists inside a ferromagnetic material. In vacuum, it leads to eq. (2), which can also be considered a good approximation for air:

$$
B=\mu_{0} \cdot H=4 \pi \cdot 10^{-7} \cdot H .
$$

In order to avoid ambiguity, the following terminology will be used: the $B$ quantity will be called "magnetic flux density", whose SI unit is the tesla (T) while the $H$ quantity will be called "magnetic field strength", whose SI unit is the ampere per meter $(\mathrm{A} / \mathrm{m})$. This follows the official terminology stated by the SI Brochure [20] and the international standard IEC 80000-6:2008: Quantities and units - Part 6: Electromagnetism. Table 1 shows the essential elements of this terminology for magnetic quantities in the SI.

Table 1- Magnetic quantities and its SI units.

\begin{tabular}{|c|c|c|c|c|}
\hline \multicolumn{2}{|c|}{ Quantity } & \multicolumn{2}{c|}{ Unit } & \multirow{2}{*}{ SI base expression } \\
\cline { 1 - 3 } Symbol & Name & Name & Symbol & \\
\hline$\phi$ & magnetic flux & weber & $\mathrm{Wb}$ & $\mathrm{m}^{2} \cdot \mathrm{kg} \cdot \mathrm{s}^{-2} \cdot \mathrm{A}^{-1}$ \\
\hline$B$ & magnetic flux density & tesla & $\mathrm{T}$ & $\mathrm{kg} \cdot \mathrm{s}^{-2} \cdot \mathrm{A}^{-1}$ \\
\hline$H$ & magnetic field strength & $\begin{array}{c}\text { ampere per } \\
\text { meter }\end{array}$ & $\mathrm{A} / \mathrm{m}$ & $\mathrm{A} / \mathrm{m}$ \\
\hline
\end{tabular}


This work seeks to use the International System of Units (SI) (maintained by the BIPM), however in the field of electromagnetism the centimeter-gram-second (CGS) system is commonly used, in particular in the specifications of magnetic transducers. Therefore, Table 2 is useful to convert units between these systems.

Table 2 - Units of magnetic quantities in the CGS system and their conversions to SI.

\begin{tabular}{|c|c|c|c|c|}
\hline \multicolumn{2}{|c|}{ Quantity } & \multicolumn{2}{c|}{ CGS Unit } & \multirow{2}{*}{ Sl conversion } \\
\cline { 1 - 4 } Symbol & Name & Name & Symbol & \\
\hline$\phi$ & magnetic flux & maxwell & $\mathrm{Mx}$ & $1 \mathrm{Mx}=10^{-8} \mathrm{~Wb}$ \\
\hline$B$ & magnetic flux density & gauss & $\mathrm{G}$ & $1 \mathrm{G}=10^{-4} \mathrm{~T}$ \\
\hline$H$ & magnetic field strength & oersted & Oe & $10 \mathrm{Oe}=10^{3} / 4 \pi \mathrm{A} / \mathrm{m}$ \\
\hline
\end{tabular}

It is worth mentioning that, in the CGS system, the permeability of free space $\mu_{0}$ is unitary. Hence, in vacuum, the magnetic flux density in $\mathrm{G}$ is numerically equal to the magnetic field strength in Oe. This is also a very good approximation for air.

1.6.

Dissertation Structure

Chapter 1: "Introduction" presents the motivation and the objectives of this dissertation, as well as providing a brief context in the most relevant international and national metrology organizations and some considerations on measurement unit systems and terminology.

Chapter 2: "Metallic Foreign Body Detection" presents a history of the research of new techniques for foreign body localization and a brief analysis of relevant and currently available high sensitivity magnetometers.

Chapter 3: "Eddy Currents" introduces the phenomenon of eddy currents induction through theoretical models obtained from classical electrodynamics and through preliminary simulation results.

Chapter 4: "Detection System for Non-Magnetic Metallic Foreign Bodies" introduces the development of an electronic system dedicated to locating nonmagnetic metallic foreign bodies for surgical removal. This includes the evolution of the design from idealization to the final version, presenting commentated 
experimental results from simulations and from practical laboratory measurements, when available.

Chapter 5: "Discussions, Conclusions and Future Works" presents the main results of the work and includes discussions on the performance of the developed device, providing possibilities for future works, including optimization of its design or promising uses of similar devices for other purposes. 


\section{2 \\ Metallic Foreign Body Detection}

\section{1. \\ History}

\subsection{1.}

\section{Locating steel needles}

An article published in 2000 described a new technique for locating steel needles in the human body using Superconducting Quantum Interference Device (SQUID) sensors [1], improving and adapting a procedure published in 1988 [21]. This technique consists in the computer acquisition of a two-dimensional magnetic field map as measured by a gradiometer using SQUID sensor elements.

The gradiometric configuration consists of two transducers, one close to the desired signal source but also exposed to the ambient noise field and another further away to detect mainly the ambient noise field, supposed to be spatially uniform. The subtraction of the signals provided by both transducers increases the signal-tonoise ratio (SNR), a necessity when measuring very weak magnetic fields in a magnetically unshielded environment.

Steel needles are ferromagnetic, therefore can present residual magnetism or be magnetized before the procedure and generate a time invariant magnetic field. In the technique described in [1], the output signal of the SQUID sensor is low-pass filtered at $20 \mathrm{~Hz}$ before being converted and acquired by a computer.

The patient is placed in a horizontal moving bed under the magnetometer, with the detection axis perpendicular to the bed. The position of the bed is monitored in the X-Y plane, associated with the gradiometer measurement of the magnetic flux density. The computer software employed plots the magnetic field map and marks the points with the highest and lowest magnetic flux density (extrema). The geometric center of these marks is also highlighted and called the magnetic center. After the completion of the mapping, these points are marked in the patient skin with a permanent marker pen, to be used as reference in the surgical removal procedure. X-ray photos were used to confirm the results before surgery. 
In [1], the localization algorithm of the developed technique is also capable of determining the depth $h$ and the angle $\alpha$ of the needle relative to the skin. To accomplish this, values are calculated for the distance $D$ between the extrema of the magnetic field intensities and for the ratio $R$ between absolute values for maximum and minimum magnetic field intensities. These were compared to curves obtained from theoretical models or in vitro data, in which the depth $h$, the angle $\alpha$ and the displacement $\Delta$ between the center of the needle and the magnetic center can be unambiguously located through association to the pair $(R, D)$. The estimated needle length should also be used to guarantee accurate magnetic field intensities in the algorithm. Figs. 3 and 4 shows these parameters in a curve of distance in the $\mathrm{x}$ axis versus the magnetic flux density in the $\mathrm{z}$ axis (normal component).

\section{skin}
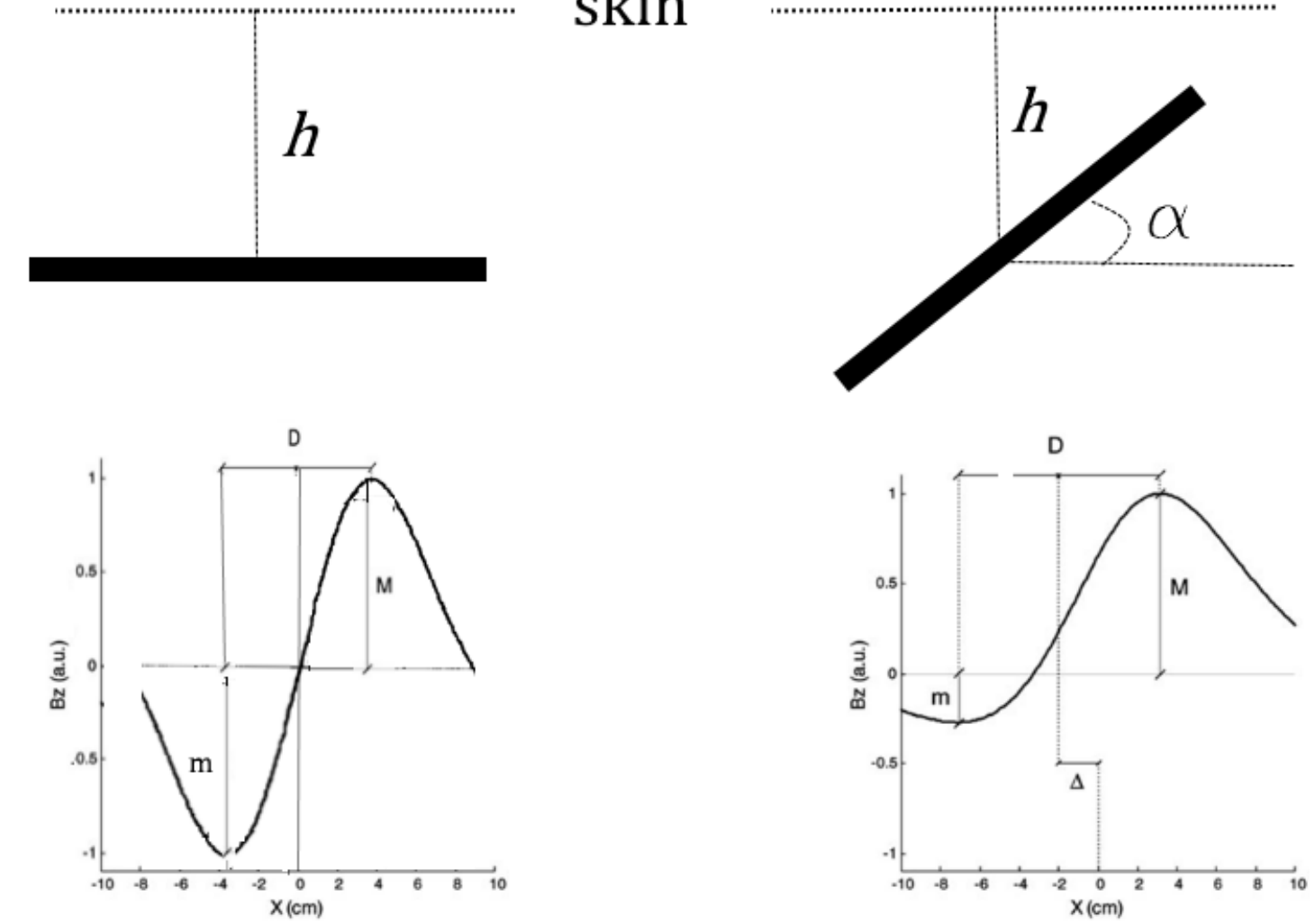

Figure 3 - Simplified diagrams showing main parameters depth $h$ and angle $\alpha$ of the needle relative to the skin. Also shown are the curves of the magnetic flux density in the $z$ axis versus the distance in the $x$ axis for two cases: $\alpha=0^{\circ}$ and $0^{\circ}<\alpha<90^{\circ}$. 


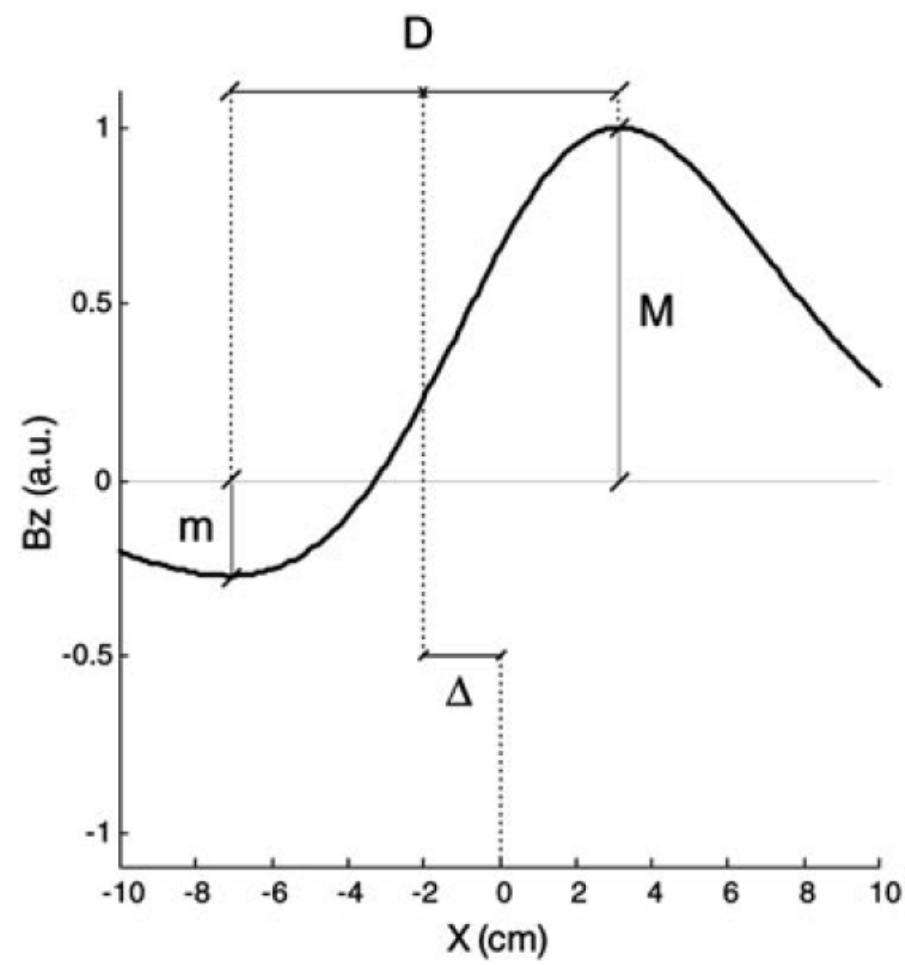

Figure 4 - Simulated normal component of the magnetic flux density generated by a $4 \mathrm{~cm}$ needle with $\alpha=30^{\circ}$ and $h=10 \mathrm{~cm}$, showing the extrema parameters $M$ and $m$, respectively the maximum and minimum magnitudes of the magnetic flux density, as well as the displacement $\Delta$ from the needle center and the position $D$ between the extrema. [1]

The technique was applied successfully to locate and surgically remove hypodermic and sewing needles in six patients. The magnetic mapping took around 5 minutes and the surgical procedures lasted between 10 to 30 minutes, which is a reduction by at least a factor of six from traditional surgical techniques. The technique also diminishes the high risk of failure of foreign body extraction. Additionally, the locating technique is innocuous and non-invasive, reducing radiation exposure for patients and staff. In the clinical studies performed, x-ray photos were used only to confirm the results of the proposed technique. Fig. 5 is a carefully performed x-ray photograph of the sixth patient, showing a very small needle fragment that was only located after the magnetic mapping was performed. 


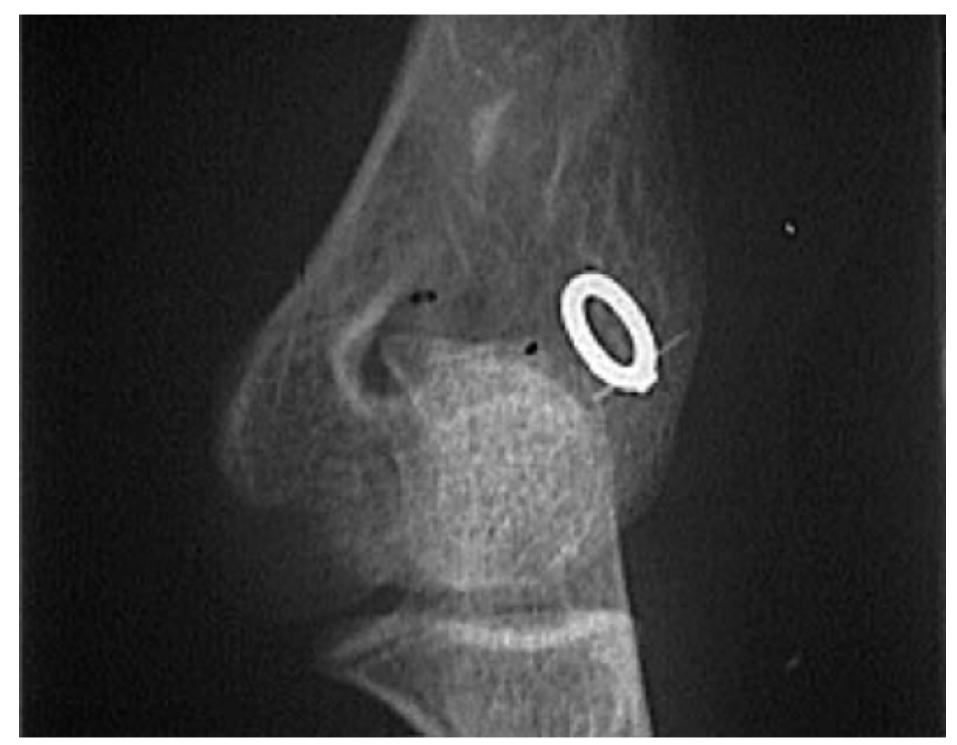

Figure 5 - X-ray film of the sixth patient indicating the steel needle location obtained by means of the procedure using SQUID magnetic mapping. The marker is above a very small steel needle fragment that, previously to the magnetic field measurement, was considered to be an artifact in the x-ray film [1].

While the technique and its surgical application were considered a success, a random DC bias variation was detected in the SQUID output, which led to errors, especially in the predicted angle $\alpha$. In 2001, an improvement of the locating technique was proposed to minimize the bias influence in measurements [2]. First, normalized magnetic field intensities are obtained after the subtraction of the average value detected. Then, new parameters are established by measuring the widths at $90 \%$ of the maximum and minimum on these normalized magnetic flux density curves in function of the distance on the needle axis, as shown in Fig. 6. The new ratio $R_{W}=W / w$ is calculated between these widths. This ratio is closely related to the angle $\alpha$, for instance a ratio of 1 would theoretically be found for a $0^{\circ}$ inclination, where the curve would be symmetrical along the $\mathrm{x}$ axis. Using these width parameters instead of the absolute magnetic flux density values would be less dependent on the DC bias of the magnetic flux density. 


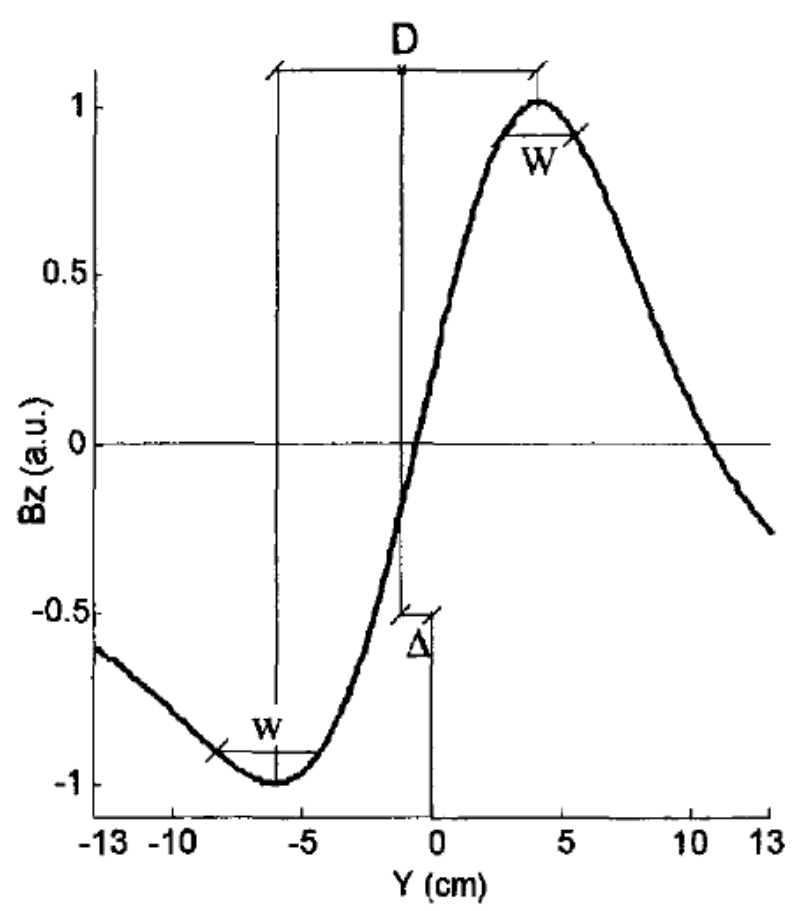

Figure 6 - Simulated normalized magnetic flux density generated by a $4.5 \mathrm{~cm}$ needle with $\alpha=40^{\circ}$ and $h=12 \mathrm{~cm}$, showing the width parameters $W$ and $w$, as well as the displacement $\Delta$ and the distance $D$ between extrema [2].

Applying this improvement to previously acquired data by comparing between the predictions and the in vitro tests showed the error in inclination prediction was reduced by more than $50 \%$.

\subsection{2.}

\section{Locating firearm projectiles}

A theoretical study was published in 2004, proposing adaptations for the steel needle location technique in order to detect one of the most prevalent foreign bodies in the modern society: the firearm projectile [3].

The main challenge in this case is that projectiles are primarily composed of lead, a diamagnetic material, which does not present a remanent magnetic field. The solution presented is to apply a time varying primary magnetic field to induce eddy currents in the projectile, since lead is a good electrical conductor. The eddy current loops generate a secondary magnetic field that can be detected by high sensitivity magnetometers. The mathematical models for eddy currents and the relation between primary and secondary magnetic field intensities used in this study are further explained in section 2.2 of the present work. The non-magnetic foreign body 
is assumed spherical for simplicity, with minimal impact on the expected magnetic field levels that depend mostly on the material characteristics and the surface area of the body.

Similar principles have been used in mine detectors. However, these devices usually compromise the spatial resolution in order to achieve a higher sensitivity. High spatial resolution is a necessary characteristic for foreign body location, since projectiles are usually small and fragmented upon impact. This, associated to the predicted secondary magnetic field intensity in the nanotesla range, leads to the selection of highly sensitive magnetometers such as fluxgate, giant magnetoresistance, giant magnetoimpedance and SQUID.

In [3], the study focused on the use of liquid helium cooled LTS SQUID, as it was the most sensitive magnetometer at the time.

Two cases are studied for the primary magnetic field generation: a solenoid or a Helmholtz coil. The latter maintains a more uniform field between its coils, but demands more space and a fixed position in relation to the patient. Mathematical results indicate similar detection capabilities; however practical considerations such as smaller dimensions and weight make the solenoid a more viable choice [3].

The magnetic field mapping is performed on a horizontal plane at a depth $h$ from the center of the sphere [3]. This magnetic field map presents cylindrical symmetry around the foreign body's rotation axis. The localization algorithm initiates with the pre-processing of measurements either through the use of a lockin amplifier or through computer acquisition and post processing to extract the inphase and quadrature signals at the primary field frequency. Fig. 7 is a simplified diagram of the proposed experimental setups.
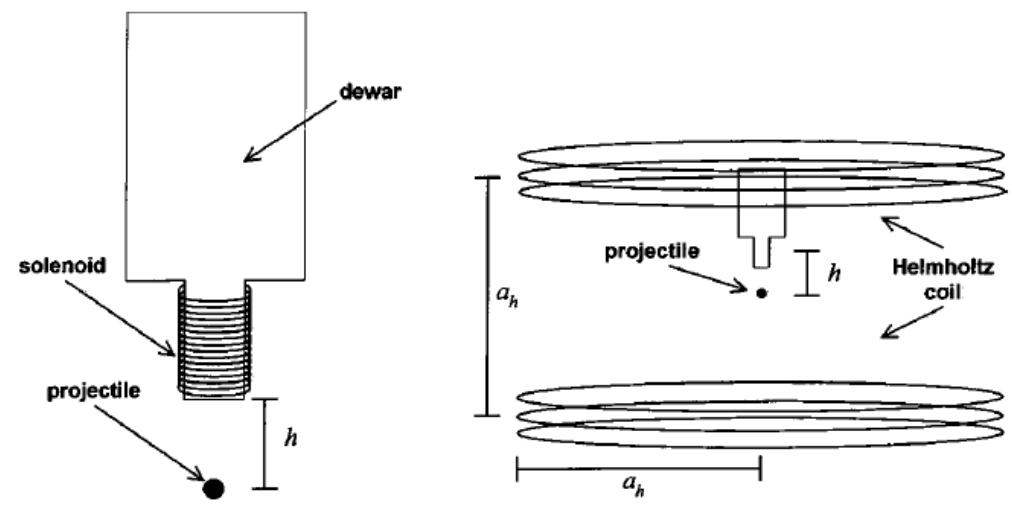

Figure 7 - Simplified diagram of two experimental setups using a SQUID dewar: using a solenoid on the left and using a Helmholtz coil on the right. [3] 
Initially, the maximum magnetic field intensity is detected. In order to minimize the effects of noise, an average of the positions weighted by its corresponding magnetic field intensity values when over $90 \%$ of the maximum is calculated and denominated the magnetic center. It indicates the horizontal position of the foreign body. The distance between the positions where the normalized magnetic field is at $90 \%$ peak value can be defined as a width parameter $w$. The relation between $h$ and $w$ is shown to be linear therefore the depth can be estimated successfully, once the magnetic center and the width are calculated.

In order to identify the radius of the foreign body, the study suggests the use of the phase difference between the primary and secondary magnetic fields. However, the relation between those parameters is heavily dependent on the frequency of the magnetic fields: on low frequencies the phase varies very little (less than $5 \%$ ) within a $1 \mathrm{~mm}$ to $15 \mathrm{~mm}$ radius range. The radius can be more successfully estimated at frequencies higher than $500 \mathrm{~Hz}$, for which the phase variation can reach $40 \%$. Fig. 8 shows simulations of measurements for magnitude and phase of the secondary magnetic flux density.
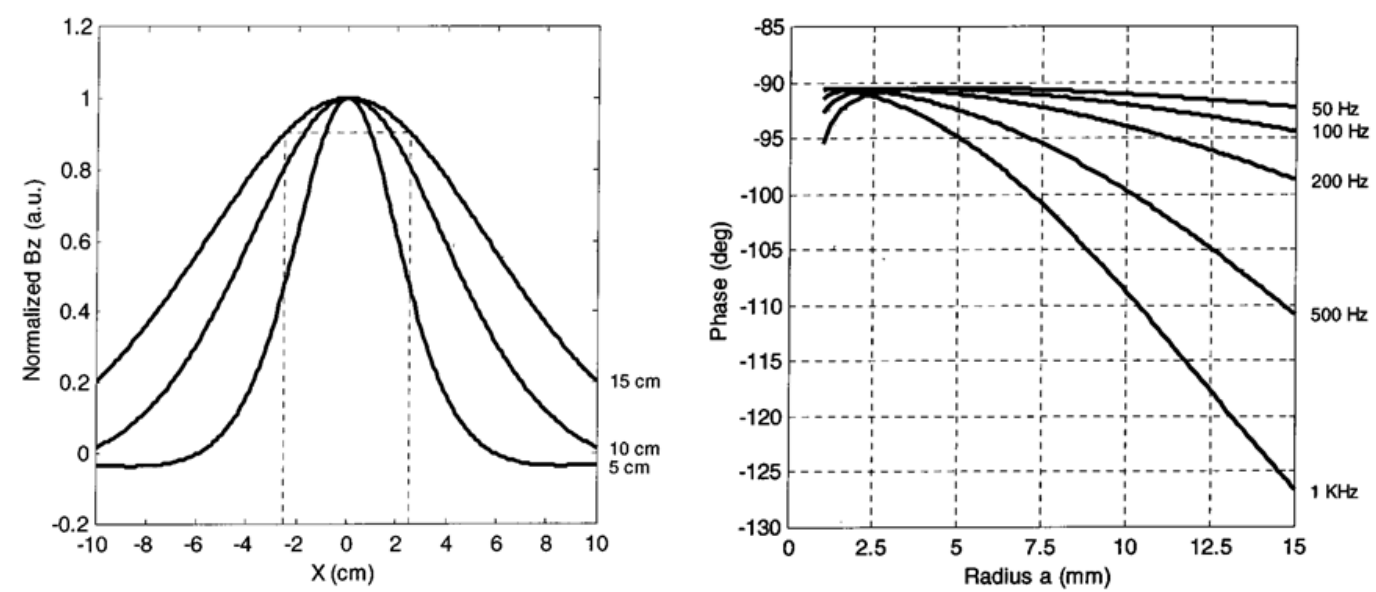

Figure 8 - Simulation results of the technique. On the left, normalized $z$ axis component of the secondary magnetic flux density $\mathrm{Bz}$ measured over the $x$ axis at the depths of 5,10 and $15 \mathrm{~cm}$. On the right, phase of the secondary magnetic flux density (in relation to primary magnetic flux density) versus the radius of the spherical foreign body, measured at frequencies of 50, 100, 200, 500 and 1000 Hz. [3] 
These graphs evidence the behavior of the magnetic flux density measurements that are used in the proposed technique. The dispersion or width of the magnitude values measured on a plane or line are related to the depth of the foreign body, while the phase is closely related to its radius.

Under the simulated results of the study, projectiles with radius as small as $3 \mathrm{~mm}$ can be located at a $10 \mathrm{~cm}$ depth, when using a SQUID based gradiometer associated with a solenoid. Fig. 9 shows simulation results of the minimum detectable radius varying with the distance, assuming the use of a solenoid and three different excitation frequencies.

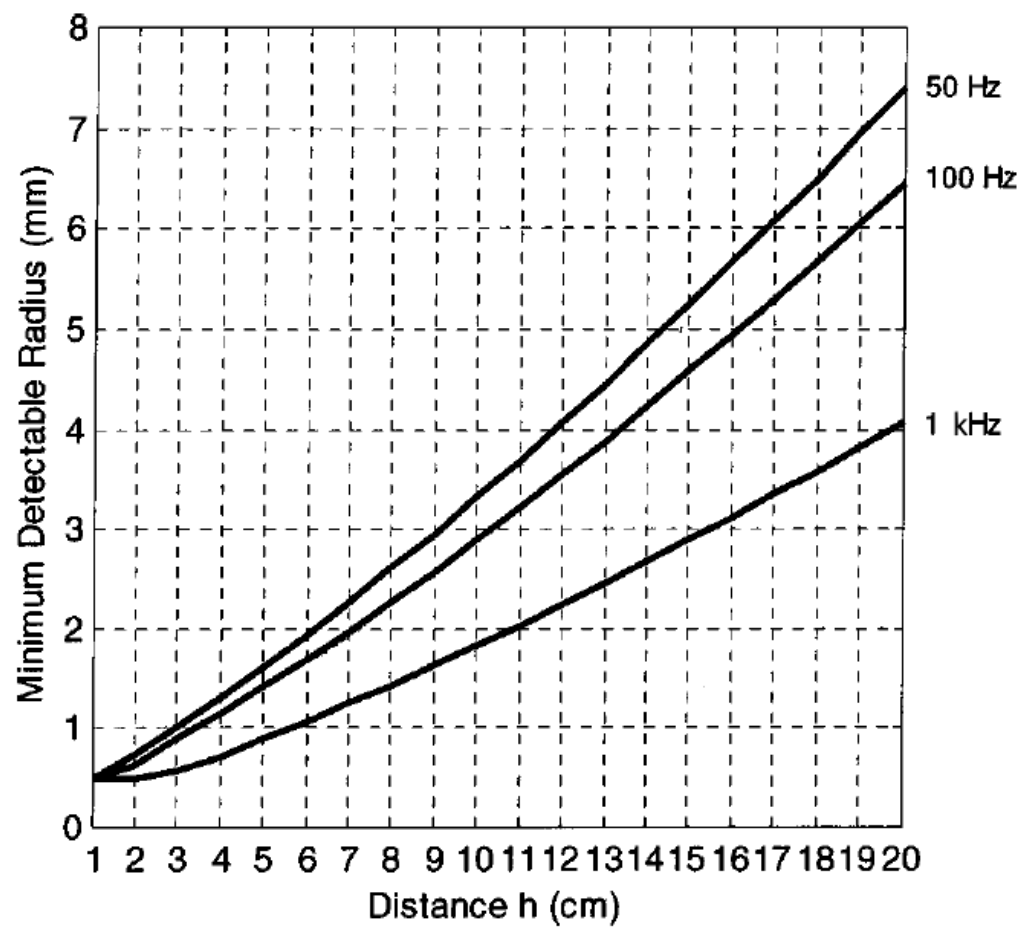

Figure 9 - Minimum detectable radius using a solenoid and a SQUID based first order gradiometer, considering a minimum detectable magnetic field of $10 \mathrm{pT}$ for each depth, for three values of the excitation frequency (50, $100 \mathrm{~Hz}$, and $1 \mathrm{kHz}$ ) [3].

\section{2.}

\section{High Sensitivity Magnetometers}

The earliest use of magnetic sensors was in the invention of the compass [22]. Further research in magnetism and magnetic measurements was only advanced in the nineteenth century. More recently, the recording and reading of data in magnetic 
storage became widespread in the information age, requiring the use of smaller and more reliable magnetic sensors.

Magnetometers are non-invasive and considered robust sensors, which makes them very useful for measurements involving electromagnetic quantities. For instance, clamp-on ammeters can measure currents without requiring contact with the conductors by detecting the magnetic fields generated by the currents. Biological measurements can also be facilitated by the use of innocuous and noninvasive magnetic readings replacing electrodes, as long as the magnetometers used have sufficient resolution and sensitivity.

There are two major types of magnetometers. Scalar magnetometers output only the magnetic field intensities without any directional information; while vector magnetometers can determine the intensities of each axis component. Proton precession magnetometers and optically pumped cesium magnetometers are the only ones that can't be used as vector magnetometers.

Since the magnetic field intensities of the secondary field in this work are expected to be in the range of nanoteslas or less and at $\mathrm{kHz}$ frequencies, only high sensitivity magnetometers with AC reading capabilities and sufficient spatial resolution (compact sensor element) have been considered. Since many works are available discussing the operating principles and advancements in magnetometers, what follows is a brief summary including only the most relevant aspects.

\subsection{1.}

\section{Superconducting Quantum Interference Device (SQUID)}

Currently, SQUIDs are the golden standard for ultra low field magnetometers, capable of achieving extremely low noise levels $(1 \mathrm{fT} / \sqrt{\mathrm{Hz}}$ for multichannel measurements under magnetically shielded chambers [23]). They can be specified to operate in wide bandwidths: from DC to RF (radio frequencies).

However, their operating principle is the use of Josephson junctions (a thin insulating barrier between two superconductor layers) which depends on very low temperatures: $4.2 \mathrm{~K}$ for low temperature superconductors (LTS) and $77 \mathrm{~K}$ for high temperature superconductors (HTS). LTS SQUIDs possess lower noise levels and require helium cooling, while HTS SQUIDs use less costly nitrogen cooling. However, the market availability of HTS SQUIDs has been gradually diminished with general consensus that LTS SQUIDs have better characteristics [23]. 
Environmental noise greatly impact SQUID measurements, requiring gradiometric configurations associated with magnetically shielded rooms covered in $\mu$-metal and aluminum, to block low-frequency and high frequency magnetic fields, respectively. This further increases costs for proper measurements.

Despite the prohibitive costs that bind SQUID uses to well-funded research institutions, they represent the state-of-the-art magnetometers to be beaten in terms of sensitivity and resolution.

Considering the use for non-magnetic metallic foreign body detection, an estimate can be obtained for the minimum detectable field [3]. Assuming a typical noise figure of $10 \mathrm{fT} / \sqrt{\mathrm{Hz}}$ for LTS SQUIDs and a frequency bandwidth of $1 \mathrm{kHz}$, the magnetic field resolution would be of $300 \mathrm{fT}$. The effect of signal-to-noise ratio of at least $30 \mathrm{~dB}$ would lead to a minimum detectable magnetic flux density of $10 \mathrm{pT}$. Since the introduced technique uses a single excitation frequency, a narrower bandwidth introduced by filters would result in even better resolution, although the noise of other circuit components could be the limiting factor.

\subsection{2.}

\section{Giant Magnetoresistance (GMR)}

The giant magnetoresistance (GMR) effect was discovered in the 1980s and rapidly became widely used, especially in reading heads for hard disk drives (HDD). They have high sensitivity and low-cost, the latter due to sharing most manufacturing technologies and techniques with semiconductors [24].

The basic physical principle of the GMR is described by quantum mechanics, more specifically, the fact that certain metals (mostly transition metals such as nickel, iron and copper) have sufficiently different energy density states for electrons of different spins at Fermi energy. The main structure used to obtain GMR effects is the spin valve: two thin magnetic films (some nanometers long) are separated by an even thinner non-magnetic film. When the magnetizations are antiparallel, there is an increased scattering due to an inversion of the state densities at the other magnetic layer, thus leading to greater resistances. When the magnetizations are parallel, state densities are spatially matched, so scattering is minimal and resistances are lower [24].

GMR sensors possess a series of advantages: high spatial resolution (compact sensor elements), high sensitivity that increases with the supply current or voltage, 
low cost and wide commercial availability. The main disadvantages are diminished effects with temperature, which also is associated to current limitations at around $10 \mathrm{~mA}$ to avoid overheating, as well as the fact that research has mostly focused on commercial applications such as reading heads and random access memory instead of ultra-low fields [23]. The study of the GMR effect also paved the way for spintronics, a new candidate for the magnetic transducers for ultra-low fields [25].

\subsection{3.}

\section{Giant Magnetoimpedance (GMI)}

The study of the giant magnetoimpedance effects began even more recently than the giant magnetoresistance. The GMI effect can be explained by classical electrodynamics, unlike the GMR effect. When a soft ferromagnetic conducting material is subjected to an alternating current at reasonably high frequencies, the skin depth is reduced and thereby the current concentrates on the surface of the material. This change in the current flow also results in variations of the impedance. With an applied external magnetic field, the magnetization increases the magnetic permeability, which affects the skin depth and, therefore, the impedance. In certain materials, this impedance variation can be of $700 \%$ for small magnetic fields (a few Oe or a few hundred $\mu \mathrm{T}$ ), characterizing the giant magnetoimpedance effect [24]. GMI elements can be as small as $1 \mu \mathrm{m}$, giving them advantages in spatial resolution and applications where miniaturization is required [24].

The variation of the impedance in GMI materials is usually symmetrical around the zero magnetic flux density, but there are ways to turn it asymmetrical (AGMI): using a DC current through the material, exposing it to AC magnetic fields or through an alteration in the manufacturing process called exchange bias. The asymmetry introduces two main advantages: the ability to distinguish the direction of a magnetic field and an increased sensitivity around the zero magnetic flux density [26].

Several studies have been realized at the LaBioMet to explore the possibilities of the GMI effect [27-33]. Sample ribbons have been provided by the Federal University of Pernambuco (UFPE). Recent studies suggested that measuring the impedance phase variation can lead to higher sensitivity than the usual impedance magnitude variation measurements [31]. Its low cost and high sensitivity are very 
advantageous, however there are some challenges in ensuring homogeneity between two samples with the same physical characteristics.

Projects were developed to characterize the sensitivity curves of GMI samples $[27,28]$, amplify and homogenize the sensitivity curves of different samples $[29,30,32]$ as well as the implementation of a functional gradiometer for measuring ultra-low magnetic fields [26]. Since the beginning, the possibility of using GMI sensors with better resolution and sensitivity for the foreign body locator has been considered [34].

The availability of a commercial prototype GMI sensor capable of measuring nanotesla levels by Aichi Micro Intelligent Corporation came as an opportunity for the design of a low cost non-magnetic metallic foreign object location system based on a sensor element capable of being produced in scale under desirable specifications. 


\section{3 \\ Eddy Currents}

Eddy currents or Foucault currents are current loops induced in conductors when subjected to time-varying magnetic fields. This is a direct consequence of the existence of a spatially-varying, non-conservative electric field, as stated by eq. (3), known as the Maxwell-Faraday equation. $\mathbf{E}$ is the electric field and $\mathbf{B}$ is the magnetic flux density. Upright boldface type denotes vector quantities.

$$
\nabla \times \mathbf{E}=-\frac{\partial \mathbf{B}}{\partial t}
$$

Eddy currents are commonly used as a non-destructive testing technique to detect cracks and flaws in metals, an important part of quality control in many sectors [35]. An often undesirable aspect of the effect is that the passing of currents through materials generate heating through the Joule effect, which can be used in induction heating. This is also responsible by power loss in devices such as transformers and electric motors, requiring consideration in order to increase efficiency.

This effect is also used in metal detectors, being the principle for the proposed device for non-magnetic metallic foreign body location. To achieve this, the parameters used must be carefully considered to align feasible primary magnetic field generation, magnetometer characteristics and biometrological principles.

\section{1.}

\section{Theoretical Formulation}

A theoretical formulation for eddy currents in conductors [5,35], has been adapted [3] for location of projectiles by magnetometers.

Considering the magnetic vector potential $\mathbf{A}$, defined as the quantity whose curl is the magnetic flux density $\mathbf{B}$, eq. (3) leads to eq. (4), where $\phi_{E}$ is the electric potential.

$$
\mathbf{E}=-\nabla \phi_{E}-\frac{\partial \mathbf{A}}{\partial t}
$$


In this study, the electric fields are generated only from the presence of a primary time-varying magnetic field, which makes $\nabla \phi_{E}=0$. The electric field $\mathbf{E}$ can be replaced by the resistivity $\rho$ and the current density $\mathbf{J}$ yielding

$$
\rho \cdot \mathbf{J}=-\frac{\partial \mathbf{A}}{\partial t}
$$

These currents flowing in a conductor of permeability $\mu$ generate a magnetic flux density given by

$$
\nabla \times \mathbf{B}=\mu \mathbf{J} .
$$

In terms of the magnetic vector potential $\mathbf{A}$, it can be written

$$
\nabla^{2} \mathbf{A}=-\mu \mathbf{J}
$$

From eqs. (5) and (6) comes

$$
\frac{\mu}{\rho} \cdot \frac{\partial \mathbf{J}}{\partial t}=-[\nabla \times(\nabla \times \mathbf{J})]=\nabla^{2} \mathbf{J}-\nabla(\nabla \cdot \mathbf{J})=\nabla^{2} \mathbf{J} .
$$

From eqs. (5) and (7),

$$
\frac{\mu}{\rho} \frac{\partial \mathbf{A}}{\partial t}=\nabla^{2} \mathbf{A}
$$

Equivalently, eq. (10) can be obtained from eqs. (5) and (6) as well as from the knowledge of Gauss law for magnetism that $\nabla \cdot \mathbf{B}=0$.

$$
\frac{\mu}{\rho} \frac{\partial \mathbf{B}}{\partial t}=-[\nabla \times(\nabla \times \mathbf{B})]=\nabla^{2} \mathbf{B}-\nabla(\nabla \cdot \mathbf{B})=\nabla^{2} \mathbf{B}
$$

Solutions for eqs. (8) and (10) can be obtained for both steady state and transient responses. For steady state solutions, the current density is usually considered the real part of a Fourier series with terms in the form $\widetilde{\mathrm{A}}_{\mathbf{n}} e^{j n \omega t}$, where $n$ is an integer, $\omega$ is the angular frequency and the inverted circumflex signifies a phasor.

\subsection{1.}

\section{Eddy Currents in Spherical Coordinates}

The projectile foreign body is modeled to be a solid lead sphere with radius $a$ much smaller than the primary magnetic field generator radius, so the field can be considered uniform. Other foreign body shapes would scale the secondary magnetic flux density by a constant factor [3]. Using spherical coordinates and assuming the primary magnetic field is independent of $\phi$ and has no $\phi$ component, the magnetic vector potential can be written as in eq. (11): 


$$
\mathbf{A}=\widehat{\boldsymbol{\phi}} A_{\phi}(r, \theta, t)
$$

The unit vector $\widehat{\boldsymbol{\phi}}$ is given by

$$
\widehat{\boldsymbol{\phi}}=-\hat{\mathbf{i}} \sin \phi+\hat{\mathbf{j}} \cos \phi,
$$

where $\hat{\mathbf{i}}$ and $\hat{\mathbf{j}}$ are unit vectors in Cartesian coordinates. The magnetic vector components in the Cartesian coordinates system are

$$
\left\{\begin{array}{c}
A_{x}=-A_{\phi} \sin \phi \\
A_{y}=A_{\phi} \cos \phi \\
A_{z}=0
\end{array}\right.
$$

Combining eq. (9) with each component in eq. (13) leads to

$$
\frac{\mu}{\rho} \widehat{\boldsymbol{\phi}} \frac{\partial A_{\phi}}{\partial t}=\nabla^{2} \mathbf{A}=\widehat{\boldsymbol{\phi}}\left(\nabla^{2} A_{\phi}-\frac{A_{\phi}}{r^{2} \sin ^{2} \theta}\right)=\widehat{\boldsymbol{\phi}} \nabla^{2} A_{\phi}+A_{\phi} \nabla^{2} \widehat{\boldsymbol{\phi}} .
$$

Laplace's equation in spherical coordinates for the electric field

$$
\nabla \cdot(\epsilon \mathrm{VE})=\frac{1}{r^{2}} \frac{\partial}{\partial r}\left(\epsilon r^{2} \frac{\partial \mathrm{V}}{\partial r}\right)+\frac{1}{r^{2} \sin \theta} \frac{\partial}{\partial \theta}\left(\epsilon \sin \theta \frac{\partial \mathrm{V}}{\partial \theta}\right)+\frac{1}{r^{2} \sin ^{2} \theta} \frac{\partial}{\partial \phi}\left(\epsilon \frac{\partial \mathrm{V}}{\partial \phi}\right)=0 .
$$

Applying eq. (15) to eq. (14) leads to

$$
\begin{gathered}
\frac{\mu}{\rho} \frac{\partial A_{\phi}}{\partial t}=\frac{1}{r^{2}} \frac{\partial}{\partial r}\left(r^{2} \frac{\partial A_{\phi}}{\partial r}\right)+\frac{1}{r^{2} \sin \theta} \frac{\partial}{\partial \theta}\left(\sin \theta \frac{\partial A_{\phi}}{\partial \theta}\right)-\frac{A_{\phi}}{r^{2} \sin ^{2} \theta}= \\
\quad=\frac{1}{r^{2}} \frac{\partial}{\partial r}\left(r^{2} \frac{\partial A_{\phi}}{\partial r}\right)+\frac{\left(1-\cos ^{2} \theta\right)^{1 / 2}}{r^{2}} \frac{\partial^{2}\left[\left(1-\cos ^{2} \theta\right)^{1 / 2} A_{\phi}\right]}{\partial \cos ^{2} \theta} .
\end{gathered}
$$

The real part of the steady state solution for a primary magnetic field with angular frequency $\omega$ is expected to be in the form of

$$
\operatorname{Re}\left[A_{\phi}\right]=\operatorname{Re}\left[\Theta r^{-1 / 2} \check{R} e^{j \omega t}\right] .
$$

After using eq. (17) in eq. (16), some manipulation leads to eq. (18), where $\sigma$ is the electrical conductivity, equal to $\rho^{-1}$ :

$$
\frac{r^{2} \mathrm{~d}^{2} \breve{R}}{\breve{R}} \frac{r}{\mathrm{~d} r^{2}}+\frac{\mathrm{d} \breve{R}}{\breve{R}} \frac{1}{\mathrm{~d} r}-\frac{1}{4}-j \mu \sigma \omega r^{2}+\frac{\left(1-\cos ^{2} \theta\right)^{1 / 2}}{\Theta} \frac{\mathrm{d}^{2}\left[\left(1-\cos ^{2} \theta\right)^{1 / 2} \Theta\right]}{\mathrm{d} \cos ^{2} \theta}=0
$$

Setting the terms involving $\theta$ equal to $-n(n+1)$ and the ones involving $r$ to $+n(n+1)$ leads to

$$
\begin{gathered}
\left(1-\cos ^{2} \theta\right) \frac{\mathrm{d}^{2} \Theta_{n}}{\mathrm{~d} \cos ^{2} \theta}-2 \cos \theta \frac{\mathrm{d} \Theta_{n}}{\mathrm{~d} \cos \theta}+\left[n(n+1)-\frac{1}{1-\cos ^{2} \theta}\right] \cdot \Theta_{n}=0 \text { and } \\
\frac{\mathrm{d}^{2} \breve{R}_{n}}{\mathrm{~d} r^{2}}+\frac{1}{r} \frac{\mathrm{d} \breve{R}_{n}}{\mathrm{~d} r}-\left[j \mu \sigma \omega+\frac{n(n+1)+1 / 4}{r^{2}}\right] \breve{R}_{n}=0 .
\end{gathered}
$$




\subsubsection{1.}

\section{Legendre's Differential Equation and Associated Legendre Functions}

The general Legendre's differential equation is

$$
\left(1-x^{2}\right) \frac{\mathrm{d}^{2} f(x)}{\mathrm{d} x^{2}}-2 x \frac{\mathrm{d} f(x)}{\mathrm{d} x}+\left[l(l+1)-\frac{m^{2}}{1-x^{2}}\right] f(x)=0 .
$$

Eq. (19) can be identified as a particular form of it for $m=1$. The general solution for eq. (21) is given in eq. (22), where $A$ and $B$ are constants while $P_{l}^{m}(x)$ and $Q_{l}^{m}(x)$ are known as associated Legendre functions of the first and second kind, respectively. The terms $l$ and $m$ are called degree and order, respectively.

$$
f(x)=A_{l} P_{l}^{m}(x)+B_{l} Q_{l}^{m}(x)
$$

For integer values of $m$ and $l$, respecting $0 \leq m \leq l$ and for real values of $\mathrm{x}$, the terms $P_{l}^{m}(x)$ become the associated Legendre polynomials. They are expressed by

$$
P_{l}^{m}(x)=\frac{(-1)^{m}}{2^{l} l !}(1-x)^{m / 2} \frac{d^{l+m}}{d x^{l+m}}\left(\left(x^{2}-1\right)^{l}\right) .
$$

The associated Legendre functions of the second kind are more complex, being defined by a series even for integer values of $m$ and $n$. Their values can be obtained from eq. (24). The same formulas can be used for functions of the first kind by replacing $Q$ for $P$.

$$
\left\{\begin{array}{c}
Q_{l}^{m}(x)=\left(1-x^{2}\right)^{m / 2} \frac{d^{m} Q_{l}(x)}{d x^{m}},-1<x<1 \\
Q_{l}^{m}(x)=\left(x^{2}-1\right)^{m / 2} \frac{d^{m} Q_{l}(x)}{d x^{m}},|x|>1
\end{array}\right.
$$

In eq. (24), $Q_{l}(x)$ are the ordinary Legendre functions of the second kind, which can be expressed through the recursion in eq. (25), using the known values presented in eq. (26):

$$
\begin{gathered}
Q_{n+1}(x)=\frac{(2 n+1) Q_{n}(x)-n Q_{n-1}(x)}{(n+1)} \\
\left\{\begin{array}{c}
Q_{0}(x)=\frac{1}{2} \ln \left(\frac{1+x}{1-x}\right) \\
Q_{1}(x)=\frac{x}{2} \ln \left(\frac{1+x}{1-x}\right)-1
\end{array}\right.
\end{gathered}
$$

Alternatively, values for $Q_{l}(x)$ can be obtained from values of $P_{l}(x)$ through the integral in eq. (27), where $P_{l}(x)$ for integer values of $l$ is given by eq. (28), known as Rodrigues' formula: 


$$
\begin{gathered}
Q_{l}(x)=P_{l}(x) \int \frac{d x}{\left(1-x^{2}\right)\left[P_{l}(x)\right]^{2}} \\
P_{l}(x)=\frac{1}{2^{l} l !} \frac{d^{l}}{d x^{l}}\left[\left(x^{2}-1\right)^{l}\right]
\end{gathered}
$$

\subsubsection{2.}

\section{Modified Bessel's Differential Equation and Modified Bessel} Functions

Eq. (29) is the modified Bessel's differential equation. Returning to eq. (20), it is identified as a particular version with $v=(j \mu \sigma \omega)^{1 / 2} r$.

$$
\frac{\mathrm{d}^{2} R_{0}}{\mathrm{~d} v^{2}}+\frac{1}{v} \frac{\mathrm{d} R_{0}}{\mathrm{~d} v}-\left[1+\frac{n^{2}}{v^{2}}\right] R_{0}=0
$$

The solution for Bessel's differential equation with complex variables follows eq. (30), where the terms $I_{n}(v)$ and $K_{n}(v)$ represent modified Bessel functions of the first and second kind, respectively. The term $n$ is the order of the functions:

$$
R_{n}^{0}(v)=C_{n} I_{n}(v)+D_{n} K_{n}(v)
$$

In the case of spherical coordinates, the order is usually half an odd integer. In these cases, the values of modified Bessel functions of the first kind can be given in terms of hyperbolic functions or in exponential form, as in

$$
I_{ \pm\left(n+\frac{1}{2}\right)}(v)=\frac{1}{(2 \pi v)^{1 / 2}} \sum_{s=0}^{n} \frac{\left[(-1)^{s} e^{v} \mp(-1)^{n} e^{-v}\right](n+s) !}{s !(n-s) !(2 v)^{s}}
$$

where $s=\sigma+j \cdot \omega$ is a complex number as used in the Laplace transform. The values of modified Bessel functions of the second kind can be obtained from

$$
K_{\left(n+\frac{1}{2}\right)}(v)=\frac{\pi}{2}(-1)^{n} \frac{I^{-\left(n+\frac{1}{2}\right)}(v)-I_{\left(n+\frac{1}{2}\right)}(v)}{\sin (n \pi)} .
$$

\subsection{2.}

\section{Steady State Solution for Eddy Currents in Spherical Coordinates}

Returning to the problem of eddy currents presented in eqs. (19) and (20), the solution for the real part of $A_{\phi}$ is given by

$$
\operatorname{Re}\left[A_{\phi}\right]=\operatorname{Re}\left[r^{-1 / 2}\left[A_{n} P_{n}^{1}(\cos \theta)+B_{n} Q_{n}^{1}(\cos \theta)\right]\left\{\check{C}_{n} I_{n+1 / 2}\left[(j \mu \sigma \omega)^{1 / 2 r}\right]+\breve{D}_{n} K_{n+1 / 2}\left[(j \mu \sigma \omega)^{1 / 2 r}\right]\right\} e^{j \omega t}\right] .
$$

For cases in which $n$ is an integer, eq. (34) is valid. That is true for $P_{n}^{1}(\cos \theta)$ and $Q_{n}^{1}(\cos \theta)$ unless conical boundaries are involved. 


$$
K_{n+1 / 2}(v)=I_{-(n+1 / 2)}(v)
$$

When conductivity is zero, $\frac{\mu}{\rho} \widehat{\boldsymbol{\Phi}} \frac{\partial A_{\phi}}{\partial t}=0$ and by making $A_{\phi} e^{j \omega t}=\check{R}^{\prime} \Theta e^{j \omega t}$ eq. (35) is obtained instead of eq. (20):

$$
\frac{\mathrm{d}}{\mathrm{d} r}\left(r^{2} \frac{d \widetilde{R^{\prime}}}{d r}\right)-n(n+1) \widetilde{R^{\prime}}=0
$$

The solution to eq. (35) is given by eq. (36):

$$
\widetilde{R}^{\prime}=\check{C} r^{n}+\check{D} r^{-n-1}
$$

Therefore, for non-conducting regions eq. (37) replaces eq. (33):

$$
\begin{aligned}
\operatorname{Re}\left[A_{\phi}\right] & =\operatorname{Re}\left[[ A _ { n } P _ { n } ^ { 1 } ( \operatorname { c o s } \theta ) + B _ { n } Q _ { n } ^ { 1 } ( \operatorname { c o s } \theta ) ] \left\{\check{C} r^{n}\right.\right. \\
+ & \left.\left.\breve{D} r^{-n-1}\right\} e^{j \omega t}\right]
\end{aligned}
$$

\subsection{3.}

\section{Eddy Currents in Conducting Spheres}

Consider a conducting sphere of radius $a$, resistivity $\rho$ and permeability $\mu$, subjected to a uniform alternating magnetic field directed along the $\mathrm{z}$ axis with magnetic flux density given by

$$
\breve{\mathbf{B}}_{\boldsymbol{p}}=\hat{\mathbf{k}} \breve{B}_{0} e^{j \omega t}
$$

where $\hat{\mathbf{k}}$ is the unit vector in the $\mathrm{z}$ axis. The phasor magnetic vector potential of the primary field is given by

$$
\check{\mathbf{A}}_{\boldsymbol{p}}=\widehat{\boldsymbol{\phi}} \frac{1}{2} \breve{B}_{0} r \sin \theta=\widehat{\boldsymbol{\Phi}} \frac{1}{2} \breve{B}_{0} r P_{1}^{1}(\cos \theta) .
$$

This specifies $n=1$ for eqs. (33) and (36), associated with boundary conditions such as the vanishing of the magnetic vector potential at infinity and the conditions within the sphere lead to

$$
\left\{\begin{array}{c}
\check{\mathbf{A}}_{o}=\widehat{\boldsymbol{\phi}} \frac{1}{2} \breve{B}_{0}\left(r+\breve{D} r^{-2}\right) \sin \theta, a<r<\infty \\
\check{\mathbf{A}}_{\boldsymbol{i}}=\widehat{\boldsymbol{\phi}} \frac{1}{2} \check{B}_{0} \check{C} r^{-1 / 2 I_{3 / 2}}\left[(j \mu \sigma \omega)^{1 / 2} r\right] \sin \theta, 0<r<a
\end{array}\right.
$$

The boundary conditions for $\mathrm{r}=a$ are in eq. (41), where $\mu_{v}$ is the permeability of a thin layer between boundaries: 


$$
\left\{\begin{aligned}
\check{\mathbf{A}}_{\boldsymbol{o}} & =\check{\mathbf{A}}_{\boldsymbol{i}} \\
\mu_{v} \frac{\partial}{\partial r}\left(r \sin \theta \check{\mathbf{A}}_{\boldsymbol{i}}\right) & =\mu \frac{\partial}{\partial r}\left(r \sin \theta \check{\mathbf{A}}_{\boldsymbol{o}}\right)
\end{aligned}\right.
$$

Using $r=a$ in eqs. (40) and (41) and using recurrence formulas for modified Bessel functions leads to

$$
\left\{\begin{array}{c}
a^{3}+\breve{D}=a^{3 / 2} \check{C} I_{3 / 2}=a^{3 / 2 \breve{C}}\left[I_{-1 / 2}-v^{-1} I_{1 / 2}\right] \\
\left(2 a^{3}-\breve{D}\right) \mu=\mu_{v} a^{3 / 2}\left[\frac{1}{2} I_{3 / 2}+v I^{\prime}{ }_{3 / 2}\right] \check{C}=\mu_{v} a^{3 / 2}\left[\left(v+v^{-1}\right) I_{1 / 2}-I_{-1 / 2}\right] \check{C}
\end{array}\right.
$$

In which $\boldsymbol{I}_{\boldsymbol{n}}$ and $\mathbf{v}$ are given by

$$
\left\{\begin{array}{c}
I_{n}=I_{n}\left[(j \mu \sigma \omega)^{1 / 2} a\right] \\
v=(j \mu \sigma \omega)^{1 / 2} a
\end{array}\right.
$$

The solution for $\breve{\boldsymbol{C}}$ and $\breve{\boldsymbol{D}}$ in eq. (42) are given by

$$
\begin{gathered}
\check{C}=\frac{3 \mu v a^{3 / 2}}{\left(\mu-\mu_{v}\right) v I_{-1 / 2}+\left[\mu_{v}\left(1+v^{2}\right)-\mu\right] I_{1 / 2}} \\
\breve{D}=\frac{\left(2 \mu+\mu_{v}\right) v I_{-1 / 2}-\left[\mu_{v}\left(1+v^{2}\right)+2 \mu\right] I_{1 / 2}}{\left(\mu-\mu_{v}\right) v I_{-1 / 2}+\left[\mu_{v}\left(1+v^{2}\right)-\mu\right] I_{1 / 2}} a^{3}
\end{gathered}
$$

Using eqs. (31) and (32), the values for the modified Bessel functions present in eqs. (44) and (45) are given by

$$
\begin{aligned}
& I_{1 / 2}(v)=\left(\frac{2}{\pi v}\right)^{\frac{1}{2}} \sinh v \\
& I_{-1 / 2}(v)=\left(\frac{2}{\pi v}\right)^{\frac{1}{2}} \cosh v
\end{aligned}
$$

The current density $\mathbf{J}$ inside the sphere is obtained by

$$
\check{\mathbf{J}}=-j \omega \sigma \check{\mathbf{A}}_{\mathbf{i}}
$$

The magnetic flux density outside the conducting sphere is given by (49) and (50).

$$
\begin{gathered}
\check{B}_{o \theta}=-\frac{1}{r} \frac{\partial}{\partial r}\left(r \check{\mathbf{A}}_{\boldsymbol{o}}\right)=-\check{B}_{0}\left(1-\frac{\breve{D}}{2 r^{3}}\right) \sin \theta \\
\check{B}_{o r}=\frac{1}{r \sin \theta} \frac{\partial}{\partial \theta}\left(\sin \theta \check{\mathbf{A}}_{\boldsymbol{o}}\right)=-\breve{B}_{0}\left(1+\frac{\breve{D}}{r^{3}}\right) \cos \theta
\end{gathered}
$$

It can be observed that the eddy current field is similar to that of a magnetic dipole loop of radius $a$, with a current flowing according to 


$$
\check{I}=\frac{2 \breve{B}_{0} \breve{D}}{\mu_{v} a^{2}} e^{j \omega t}
$$

In order to obtain results for a static primary magnetic field $(\omega=0)$, the following relations are useful:

$$
\left\{\begin{array}{l}
I_{1 / 2}(x) \stackrel{x \rightarrow 0}{\rightarrow}\left(\frac{2}{\pi x}\right)^{1 / 2}\left(x+\frac{x^{3}}{6}\right) \\
I-1 / 2(x) \stackrel{x \rightarrow 0}{\rightarrow}\left(\frac{2}{\pi x}\right)^{1 / 2}\left(1+\frac{x^{2}}{2}\right)
\end{array}\right.
$$

Using eq. (52) to simplify results for $\check{C}$ and $\breve{D}$ at eqs. (44) and (45), and these in eq. (40), we obtain eq. (53), which represents the phasor magnetic vector potential for a static primary magnetic field:

$$
\left\{\begin{array}{c}
\check{\mathbf{A}}_{\boldsymbol{o}}=\widehat{\boldsymbol{\phi}} \frac{\breve{B}_{0}}{2}\left[r+\frac{2\left(K_{m}-1\right) a^{3}}{\left(K_{m}+2\right) r^{2}}\right] \sin \theta, a<r<\infty \\
\check{\mathbf{A}}_{\boldsymbol{i}}=\widehat{\boldsymbol{\Phi}} \frac{3 K_{m} \check{B}_{0}}{2\left(K_{m}+2\right)} r \sin \theta, 0<r<a
\end{array}\right.
$$

Using eq. (48), the first approximation for slowly alternating fields is introduced in

$$
\check{J}_{\phi}=-\frac{3 j \omega \sigma K_{m} \check{B}_{0}}{2\left(K_{m}+2\right)} r \sin \theta .
$$

When frequency tends to infinity, the following would be valid:

$$
I_{1 / 2}(x) \stackrel{x \rightarrow \infty}{\longrightarrow} I_{3 / 2}(x) \stackrel{x \rightarrow \infty}{\longrightarrow} I_{-1 / 2}(x) \stackrel{x \rightarrow \infty}{\longrightarrow}\left(\frac{2}{\pi x}\right)^{1 / 2}\left(\frac{e^{x}}{2}\right) .
$$

This would lead to

$$
\left\{\begin{array}{c}
\check{\mathbf{A}}_{o} \rightarrow \widehat{\boldsymbol{\Phi}} \frac{\check{B}_{0}}{2}\left(r-a^{3} r^{-2}\right) \sin \theta, a<r<\infty \\
\check{\mathbf{A}}_{i} \rightarrow 0,0<r<a
\end{array}\right.
$$

These results suggest there are no magnetic fields inside the sphere, so the eddy currents occur only at the surface of the sphere, as it is expected at high frequencies. 


\subsection{4.}

\section{Eddy Currents Calculations for Magnetometers}

The results for the secondary magnetic vector potential component $A_{s \phi}$ at eq. (40) can be rewritten [35] using eqs. (45 - 47), subtracting the primary magnetic vector potential $A_{p \phi}$ and assuming $\mu_{v} \approx \mu_{o}$ to reach eq. (57), where $\mu_{r}=\frac{\mu}{\mu_{o}}$ is the relative permeability of the conducting sphere.

$$
A_{s \phi}(r, \theta, \phi)=\frac{1}{2} B_{o}\left[a^{3} \frac{\left(2 \mu_{r}+1\right)-\left(2 \mu_{r}+v^{2}+1\right) \frac{\tanh v}{v}}{\left(\mu_{r}-1\right)-\left(\mu_{r}-v^{2}+1\right) \frac{\tanh v}{v}}\right]\left(\frac{\sin \theta}{r^{2}}\right)
$$

The parameter $\boldsymbol{v}$, introduced in eq. (43) can also be expressed as

$$
v=(1+j) \frac{a}{\delta}
$$

where $\delta$ is the eddy current skin depth, given by

$$
\delta=\sqrt{\frac{1}{f_{o}} \frac{1}{\pi \mu_{r} \mu_{o} \sigma}}
$$

where $f_{o}=\omega / 2 \pi$ is the frequency of the primary magnetic field. In eq. (57), the term $\left(\frac{\sin \theta}{r^{2}}\right)$ is the same present in a pure dipole field while the term between the brackets is a constant depending on material parameters and excitation frequency. This suggests the effect of finite dimensions and different frequencies affects the secondary magnetic field in the form of a multiplication factor to the response of an infinitesimal sphere to a static field.

The magnetic flux density can be obtained by taking the curl of the magnetic vector potential, that is,

$$
\begin{aligned}
\mathbf{B}_{\boldsymbol{s}}(r, \theta, \phi)= & \boldsymbol{\nabla} \times \mathbf{A}_{\boldsymbol{s}}(r, \theta, \phi) \\
& =\frac{1}{r \sin \theta} \frac{\partial}{\partial \theta}\left[\frac{\partial}{\partial \theta}\left(A_{s \phi} \sin \theta\right)-\frac{\partial A_{s \theta}}{\partial \phi}\right] \widehat{\mathbf{r}} \\
& +\frac{1}{r}\left[\frac{1}{\sin \theta} \frac{\partial A_{s r}}{\partial \phi}-\frac{\partial\left(r A_{s \phi}\right)}{\partial r}\right] \widehat{\boldsymbol{\theta}} \\
& +\frac{1}{r}\left[\frac{\partial\left(r A_{s \theta}\right)}{\partial r}-\frac{\partial A_{s r}}{\partial \theta}\right] \widehat{\boldsymbol{\phi}}
\end{aligned}
$$

Since in this case there is only a $\phi$ component to the magnetic vector potential, eq. (60) becomes 


$$
\mathbf{B}_{s}(r, \theta, \phi)=\frac{1}{r \sin \theta} \frac{\partial}{\partial \theta}\left[\frac{\partial}{\partial \theta}\left(A_{s \phi} \sin \theta\right)\right] \hat{\mathbf{r}}+\frac{1}{r}\left[\frac{\partial\left(r A_{s \phi}\right)}{\partial r}\right] \widehat{\boldsymbol{\theta}}+0 \widehat{\boldsymbol{\phi}} .
$$

After using eq. (57) in eq. (61), the components of the magnetic flux density in spherical coordinates are given by

$$
\begin{gathered}
B_{s r}(r, \theta, \phi)=B_{o} a^{3}\left[\frac{\left(2 \mu_{r}+1\right)-\left(2 \mu_{r}+v^{2}+1\right) \frac{\tanh v}{v}}{\left(\mu_{r}-1\right)-\left(\mu_{r}-v^{2}+1\right) \frac{\tanh v}{v}}\right]\left(\frac{\cos \theta}{r^{3}}\right), \\
B_{s \theta}(r, \theta, \phi)=\frac{B_{o}}{2} a^{3}\left[\frac{\left(2 \mu_{r}+1\right)-\left(2 \mu_{r}+v^{2}+1\right) \frac{\tanh v}{v}}{\left(\mu_{r}-1\right)-\left(\mu_{r}-v^{2}+1\right) \frac{\tanh v}{v}}\right]\left(\frac{\sin \theta}{r^{3}}\right) \text { and } \\
B_{s \phi}(r, \theta, \phi)=0 .
\end{gathered}
$$

The component of the magnetic flux density in the $\mathrm{z}$ axis of Cartesian coordinates is calculated by

$$
B_{s z}(r, \theta, \phi)=B_{s r}(r, \theta, \phi) \cos \theta-B_{s \phi}(r, \theta, \phi) \sin \theta,
$$

resulting in

$$
B_{S Z}(r, \theta, \phi)=\frac{B_{o}}{2} a^{3}\left[\frac{\left(2 \mu_{r}+1\right)-\left(2 \mu_{r}+v^{2}+1\right) \frac{\tanh v}{v}}{\left(\mu_{r}-1\right)-\left(\mu_{r}-v^{2}+1\right) \frac{\tanh v}{v}}\right]\left(\frac{1}{r^{3}}\right)\left(3 \cos ^{2} \theta-1\right) .
$$

The relations in eq. (67) are used in order to obtain parameters in Cartesian coordinates, where $h$ is the distance between the sphere and the plane of measurement, also called depth.

$$
\left\{\begin{aligned}
r & =\sqrt{x^{2}+y^{2}+h^{2}} \\
\cos \theta & =\frac{h}{r}=\frac{h}{\sqrt{x^{2}+y^{2}+h^{2}}}
\end{aligned}\right.
$$

Using the relations in eq. (67) leads to

$$
B_{S Z}(x, y, h)=\frac{B_{o}}{2} a^{3}\left[\frac{\left(2 \mu_{r}+1\right)-\left(2 \mu_{r}+v^{2}+1\right) \frac{\tanh v}{v}}{\left(\mu_{r}-1\right)-\left(\mu_{r}-v^{2}+1\right) \frac{\tanh v}{v}}\right]\left(\frac{2 h^{2}-x^{2}-y^{2}}{\left(x^{2}+y^{2}+h^{2}\right)^{5 / 2}}\right) .
$$

This magnetic flux density possesses cylindrical symmetry, with peak value $B_{\text {smax }}$ at $x=y=0$. This parameter is very useful to choose magnetometer characteristics and can be calculated from

$$
B_{\text {smax }}\left(h, a, f_{o}\right)=B_{o} a^{3}\left[\frac{\left(2 \mu_{r}+1\right)-\left(2 \mu_{r}+v^{2}+1\right) \frac{\tanh v}{v}}{\left(\mu_{r}-1\right)-\left(\mu_{r}-v^{2}+1\right) \frac{\tanh v}{v}}\right]\left(\frac{1}{h^{3}}\right) .
$$


This equation can be rewritten by naming the complex multiplication factor as $V\left(a, f_{o}\right)$, such as

$$
V\left(a, f_{o}\right)=a^{3}\left[\frac{\left(2 \mu_{r}+1\right)-\left(2 \mu_{r}+v^{2}\left(a, f_{o}\right)+1\right) \frac{\tanh v\left(a, f_{o}\right)}{v\left(a, f_{o}\right)}}{\left(\mu_{r}-1\right)-\left(\mu_{r}-v^{2}\left(a, f_{o}\right)+1\right) \frac{\tanh v\left(a, f_{o}\right)}{v\left(a, f_{o}\right)}}\right] .
$$

Then, eq. (69) becomes eq. (71), which separates the elements varying with the characteristics of the sphere and the excitation from the effect of the depth.

$$
B_{\text {smax }}\left(h, a, f_{o}\right)=\left(\frac{B_{o}}{h^{3}}\right) V\left(a, f_{o}\right)
$$

Decomposing $V\left(a, f_{o}\right)$ exhibits the effect on magnitude and phase of the secondary magnetic flux density peak in relation to the primary magnetic flux density, as seen in

$$
\frac{B_{\text {smax }}\left(h, a, f_{o}\right)}{B_{o}}=\frac{\left|V\left(a, f_{o}\right)\right| e^{j \varphi\left(a, f_{o}\right)}}{h^{3}} .
$$

\subsection{5.}

\section{Gradiometer effects}

In order to greatly improve signal-to-noise ratio by minimizing the effect of environmental magnetic fields in the measurements, the magnetometer should utilize a gradiometric (differential) configuration. The two sensor elements are separated by a distance $l_{s}$, called the base line. The detected secondary magnetic flux density for a first-order gradiometer is presented in eq. (73), under the supposition that the environmental field is successfully nullified.

$$
B_{s z}(x, y, h)=\frac{B_{o}}{2} V\left(a, f_{o}\right)\left(\frac{2 h^{2}-x^{2}-y^{2}}{\left(x^{2}+y^{2}+h^{2}\right)^{5 / 2}}-\frac{2\left(h+l_{s}\right)^{2}-x^{2}-y^{2}}{\left(x^{2}+y^{2}+\left(h+l_{s}\right)^{2}\right)^{5 / 2}}\right)
$$

The peak value is shown in given by

$$
B_{\text {smax }}\left(h, a, f_{o}\right)=B_{o} V\left(a, f_{o}\right)\left(\frac{1}{h^{3}}-\frac{1}{\left(h+l_{s}\right)^{3}}\right) \text {. }
$$




\section{2.}

\section{Simulated Results and Preliminary Conclusions for Excitation Parameters}

The parameters $a, \mu_{r}, \sigma$ and $h$ cannot be controlled, since they depend on the characteristics of the foreign body and its position within the patient. The baseline also depends heavily on the device configuration, especially the characteristics of the primary magnetic field generator. Therefore, only the frequency and the primary magnetic flux density can be truly specified in order to match sensor characteristics.

In the case of lead projectiles, the material parameters are:

$$
\left\{\begin{array}{c}
\sigma=4.55 \times 10^{6} \mathrm{~S} / \mathrm{m} \\
\mu_{r}=1-1.7 \times 10^{-5} \\
\mu=\mu_{r} \mu_{o}=1.26 \times 10^{-6} \mathrm{Tm} / \mathrm{A}
\end{array} .\right.
$$

Based on the mathematical development presented, eq. (69) was used in simulations for secondary magnetic fields generated by eddy currents in lead spherical foreign bodies with radii between $1 \mathrm{~mm}$ and $5 \mathrm{~mm}$ at depth $10 \mathrm{~cm}$ result in the curves for relative peak magnetic flux density $\left(B_{\text {smax }} / B_{0}\right)$ magnitude and phase in function of the frequency $f_{o}$ shown in Fig. 10. The respective radii values for each curve are shown at right.
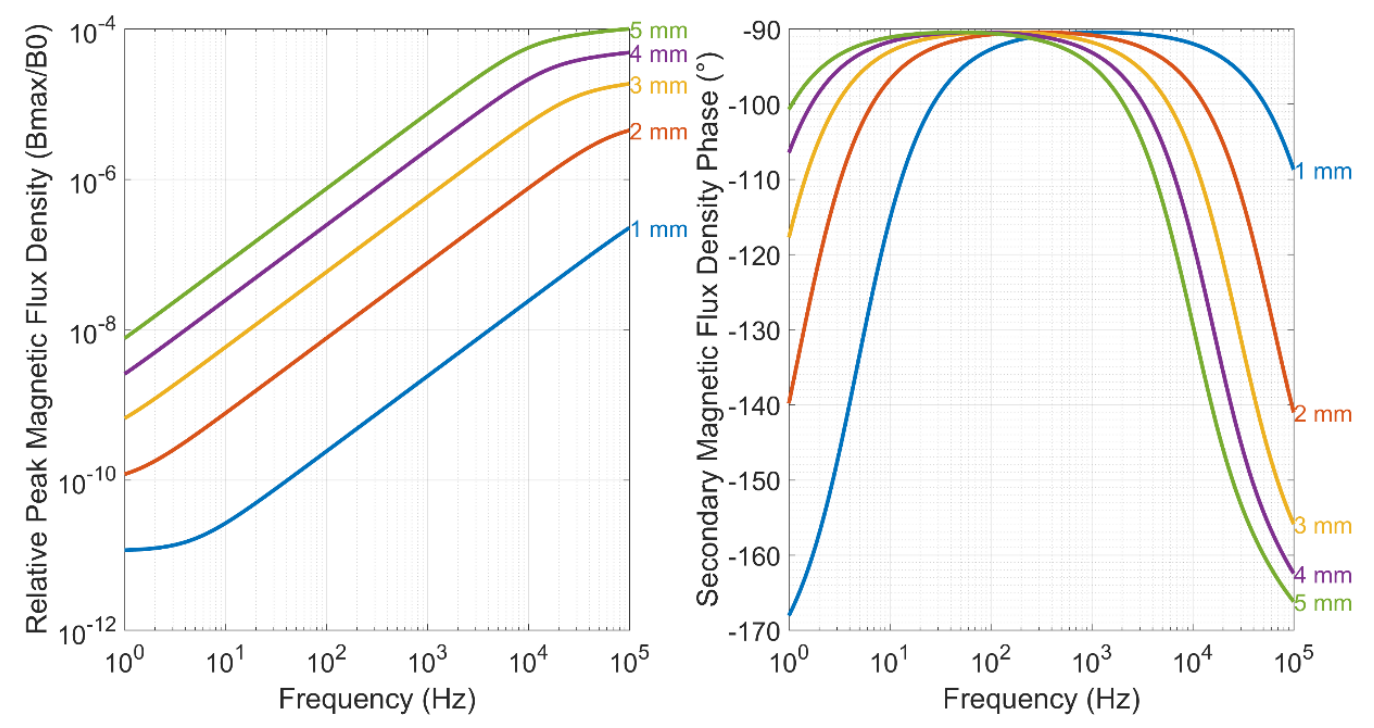

Figure 10 - Simulated results for secondary magnetic fields due to eddy currents at a spherical foreign body made of lead with radii between $1 \mathrm{~mm}$ and $5 \mathrm{~mm}$ at a depth $h=10 \mathrm{~cm}$, presented in relative peak magnetic flux density $\left(B_{\text {smax }} / B_{0}\right)$ amplitude and phase in function of the frequency $f_{o}$. The respective radii for each curve are shown at right. 
Regarding the magnitude, it can be observed that higher frequencies produce larger secondary magnetic flux densities, as expected. The relation is almost linear for a long range, but there is a maximum around $100 \mathrm{kHz}$ when the relative peak magnetic flux density tends to become constant. The ratio between the secondary and the primary magnetic flux density still shows how the secondary values are much weaker, requiring a high sensitivity magnetic transducer. The radius of the foreign object also affects the magnitude with higher radii leading to greater secondary magnetic flux densities.

The phase results are even more frequency dependent, with the phase for any radii changing between $-90^{\circ}$ and $-170^{\circ}$. The frequency response is shifted towards lower frequencies for higher radii.

Preliminary conclusions from these more generalized simulations are that using excitation frequencies near $100 \mathrm{kHz}$ and the highest possible primary magnetic flux densities would benefit measurements. However, these parameters are limited by a few practical considerations: the ICNIRP guidelines for acceptable magnetic fields for humans, the capability of generating strong magnetic fields at high frequency and the linear operating range of the magnetic transducers. 

Bodies

The location system is fundamentally composed of two major components: the primary magnetic field generator and the signal processing circuit. The interface between them is the pair of sensor elements for gradiometric measurements.

The primary magnetic field generator is a solenoid and its power supply. The signal processing is performed by analog electronics that filter and amplify the signals, as well as perform the gradiometer's subtraction between the two sensor outputs. The focus of this work is the development, implementation and simulation test of this electronic system. Afterwards, the final output of this electronic circuit can be subjected to computer acquisition followed by post-processing. Fig. 11 introduces the outline of this detection system presented in block diagram form:

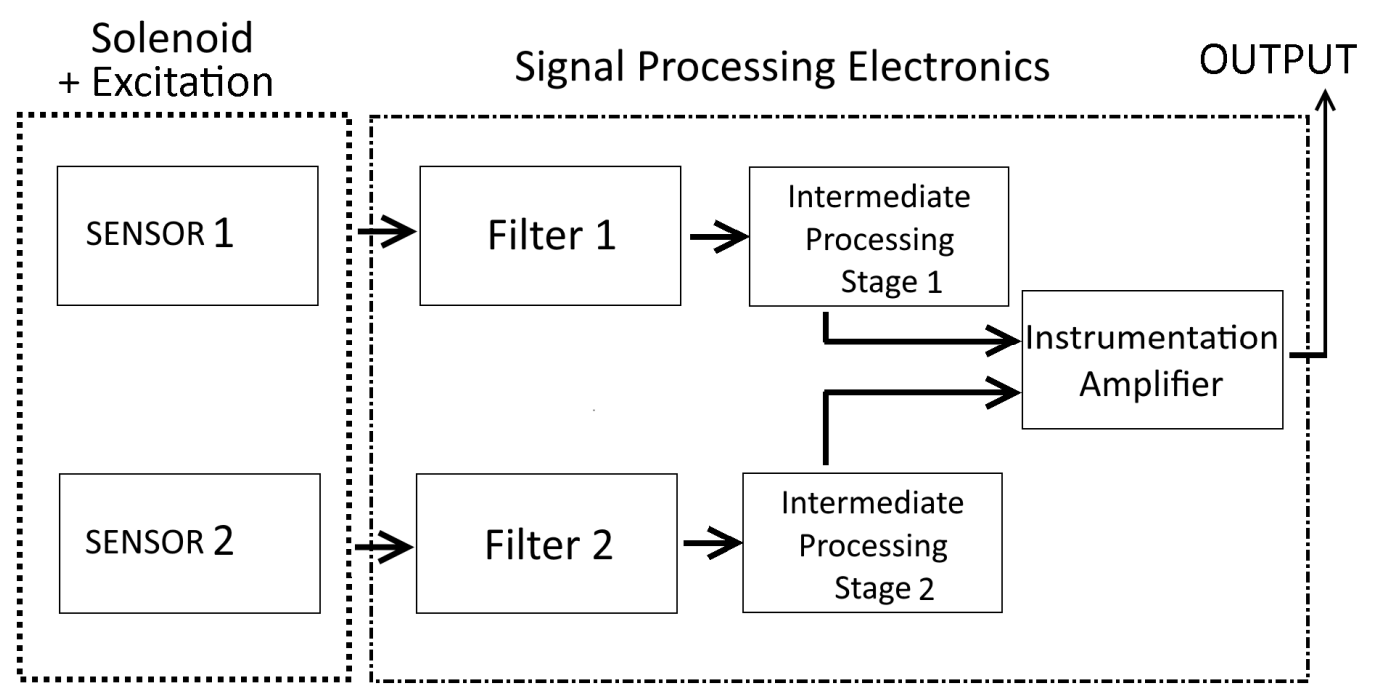

Figure 11 - Block diagram of a generic foreign body detection system circuit. 
In the projects developed, each sensor output is initially filtered and then passes through an optional intermediate processing stage. This stage can be adapted to the necessities of a particular sensor element or topology, for example introducing an initial gain adjustment to correct filter gain mismatches or providing signal rectification. Finally, the processed signals originated from each sensor serve as inputs to an instrumentation amplifier that subtracts the signals and introduces a large differential gain, providing the gradiometric reading at the output.

Since diverse sensor elements differ in several key characteristics, the entire system needs to be reworked, especially the primary magnetic field generator, due to differences in linear operation range and bandwidth. Therefore, this chapter splits the GMR projects from the GMI projects. All projects use the INA129 instrumentation amplifier. A few considerations on its properties can be found in Appendix A. What follows is a description of the most relevant projects in chronological order, commenting the simulation and experimental results obtained.

\section{1. \\ Developments Based on GMR Sensors}

\subsection{1.}

\section{Model Used: NVE AA-005-002}

Initial studies for the non-ferromagnetic metallic foreign body location system used the GMR sensor NVE AA-005-002. It possess a minimum sensitivity of $0.45 \mathrm{mV} / \mathrm{V} / \mathrm{Oe}=5.65 \mu \mathrm{V} /\left(\mathrm{V} . \mathrm{A} \cdot \mathrm{m}^{-1}\right)$ (approximately $4.5 \mathrm{~V} / \mathrm{V} / \mathrm{T}$ in free space) for the linear operation range for magnetic field intensities between 10 Oe and 70 Oe (approximately $1 \mathrm{mT}$ to $7 \mathrm{mT}$ at free space permeability) with a saturation field of $100 \mathrm{Oe}$. The maximum sensitivity is $0.65 \mathrm{mV} / \mathrm{V} / \mathrm{Oe}$. The maximum input voltage range is $24 \mathrm{~V}$. This would suggest an upper limit for the final sensitivity at 15.6 $\mathrm{mV} / \mathrm{Oe}(156 \mathrm{~V} / \mathrm{T})$.

This GMR sensor can operate in frequencies from DC to slightly above $1 \mathrm{MHz}$ and temperatures from $-50{ }^{\circ} \mathrm{C}$ up to $125^{\circ} \mathrm{C}$, however higher temperatures can affect the linear range. Using a current supply instead of a voltage supply ensures a better temperature performance, according to its datasheet.

This transducer is presented in a SOIC8 package with the size of $411 \mu \mathrm{m}$ by $1458 \mu \mathrm{m}$. Each package is disposed in a Wheatstone Bridge configuration with four 
GMR sensors. One pair is shielded from external magnetic fields while the other pair is subject to its effects. Each resistance is specified as $5 \mathrm{k} \Omega \pm 20 \%$. The voltage sensitivity of 0.45 to $0.55 \mathrm{mV} / \mathrm{V} / \mathrm{Oe}$ corresponds to a resistance sensitivity of 4.5 to $5.5 \Omega / \mathrm{Oe}$ (independent of the voltage supply).

Characterization studies were performed using this GMR package in a gradiometric measurement circuit and resulted in an estimated resolution of $43 \mu \mathrm{Oe}$, or $4.3 \mathrm{nT}$ in air [36], however these studies used a very narrow bandwidth between DC and $5 \mathrm{~Hz}$ due to the use of peak detectors and low-pass filters. Furthermore, it was assumed that the noise was predominantly electronic in nature and primarily due to the signal processing components. Considering the instrumentation amplifiers used have very low noise voltage levels and the bandwidth is very small, it can be inferred that this is not necessarily the case. By otherwise assuming the predominant noise to be originating from the sensor package an estimated resolution for the GMR can be given. This would be a best case scenario value for any device using this sensor.

Under this condition that the noise is originating from the GMR sensor, a theoretically applied final gain of 100 times would not be capable of altering this resolution, thus the value for this narrow bandwidth would be $4.3 \mathrm{mOe}$, or $430 \mathrm{nT}$ in air. Knowing the primary noise component in this type of magnetic sensor follows a $1 / f$ spectrum, an estimate of the noise can be obtained for any bandwidth. First, the power for a given noise voltage value is

$$
S_{\text {noise }}=\frac{v_{\text {noise rms }}{ }^{2}}{1 \Omega}=\frac{(\text { Res } \cdot \text { Sens })^{2}}{1 \Omega},
$$

where $S_{\text {noise }}$ is the power of the noise, $v_{\text {noise }}$ is the root mean square amplitude of the noise voltage, Res is the sensor resolution and Sens is its sensitivity. It is customary to consider the output impedance to be $1 \Omega$ to simplify calculations. Noise can be modeled by a stochastic process, but the voltage found experimentally in [36] was peak-to-peak. This value can be considered as the upper limit for a confidence interval with $99.9 \%$ certainty, which, considering a Gaussian distribution, can be used to estimate the rms value of the stochastic process (standard deviation) by using a factor of 6.6. Using the experimentally measured GMR noise and its selected sensitivity yields

$$
S_{n G M R}=\frac{\left(430 / 6.6 \cdot 10^{-9} \mathrm{~T} \cdot 22.5 \mathrm{~V} / \mathrm{T}\right)^{2}}{1 \Omega} \approx \frac{\left(1.466 \cdot 10^{-6} \mathrm{~V}\right)^{2}}{1 \Omega} \approx 2.15 \mathrm{pW} .
$$


Then, $S_{n G M R}$ is taken to be the result of the integral of the noise spectral power density curve equation over the frequency bandwidth, as shown in eq. (78). The noise spectral power density curve is assumed to follow a $1 / \mathrm{f}$ behavior, so it is modeled by $S_{G M R o} / f$, where $S_{G M R o}$ is the spectral noise power density at $1 \mathrm{~Hz}$ and $f$ is the frequency. The use of DC signals can be a problem for the simplified model used, but 1/f noise close to DC values can be similar to drift and, although high in value, it is easier to distinguish from the signal of interest. The peak-to-peak noise value obtained was already considering $\mathrm{AC}$ noise only, so a safe assumption would be the lower limit of the bandwidth to be $1 \mathrm{~Hz}$. The highest frequency is the lowpass filter cutoff frequency of around $5 \mathrm{~Hz}$ adjusted by the factor of 1.57 to compose the Equivalent Noise Bandwidth for a first order RC low pass filter.

$$
\int_{1}^{1.57 \cdot 5} \frac{S_{G M R o}}{f} d f=S_{G M R o} \cdot \ln \left(\frac{1.57 \cdot 5}{1}\right)=S_{n G M R} \approx 2.15 \mathrm{pW}
$$

The resulting parameter for spectral noise power density at $1 \mathrm{~Hz}\left(S_{G M R o}\right)$ is given by

$$
S_{G M R o} \approx 1.05 \frac{\mathrm{pW}}{\mathrm{Hz}}
$$

Reverting it back to a magnetic flux density unit (the noise density) leads to

$$
\text { Noise }_{\text {GMRo }}=\frac{\sqrt{S_{G M R o}}}{\operatorname{SenS}}=\frac{\sqrt{1.05 \cdot 10^{-12}}}{22.5} \approx 45.4 \frac{\mathrm{nT}}{\sqrt{\mathrm{Hz}}} \text {. }
$$

This is quite higher than what SQUID sensors could perform, but can be sufficient for larger foreign bodies at smaller distances. The sensitivity obtained from experimental results was at the minimum specified of $0.45 \mathrm{mV} / \mathrm{V} / \mathrm{Oe}$ or $4.5 \mathrm{~V} / \mathrm{V} / \mathrm{T}$.

Many of these attributes are essential for the design of the locating system, so for easy reference they are summed up in Table 3. 
Table 3- Main characteristics for GMR sensor NVE AA-005-02.

\begin{tabular}{|c|c|c|c|}
\hline GMR characteristics & Minimum & Maximum & Unit \\
\hline $\begin{array}{c}\text { Linear Range } \\
\text { (in air) }\end{array}$ & 10 & 70 & Oe \\
\cline { 2 - 4 } Sensitivity & 1 & 7 & $\mathrm{mT}$ \\
\hline (in air) & 0.45 & 0.65 & $\mathrm{mV} /(\mathrm{V} \cdot \mathrm{Oe})$ \\
\hline $\begin{array}{c}\text { Saturation Field } \\
\text { (in air) }\end{array}$ & 4.5 & 6.5 & $\mathrm{~V} /(\mathrm{V} \cdot \mathrm{T})$ \\
\hline Nominal Resistance & $5-20 \%$ & $5+20 \%$ & $\mathrm{k} \Omega$ \\
\hline Input Voltage Range & $<1$ & 24 & $\mathrm{~V}$ \\
\hline Operating Frequency & $\mathrm{DC}$ & $>1$ & $\mathrm{MHz}$ \\
\hline Operating Temperature & -50 & 125 & ${ }^{\circ} \mathrm{C}$ \\
\hline Bridge Electrical Offset & -4 & 4 & $\mathrm{mV} / \mathrm{V}$ \\
\hline Estimated noise density & 454 & & $\mu \mathrm{Oe} / \sqrt{\mathrm{Hz}}$ \\
\cline { 2 - 4 } (in air) & 45.4 & & $\mathrm{nT} / \sqrt{\mathrm{Hz}}$ \\
\hline
\end{tabular}

\subsection{2.}

\section{Primary Magnetic Field Generator Design}

The primary magnetic field generator is a solenoid and its peak magnetic flux density is approximated by

$$
B_{\text {sol_peak }}=\mu_{o} \mu_{r} \frac{N I}{L}
$$

where $N$ is the number of turns, $I$ is the current flowing through the solenoid and $L$ is the solenoid length. The theoretical value of this magnetic flux density along the axis of the solenoid for any distance can be obtained by

$$
B_{\text {sol }}=\mu_{o} \mu_{r} \frac{N I}{2 L}\left(\frac{D+L}{\sqrt{(D+L)^{2}+R^{2}}}-\frac{D}{\sqrt{D^{2}+R^{2}}}\right),
$$

where $R$ is the solenoid radius and $D$ is the distance in the $\mathrm{z}$ axis, considering one edge of the solenoid as the origin.

The linear operation range of the chosen GMR sensor is between 10 Oe and 70 Oe, or equivalently in air from $1 \mathrm{mT}$ to $7 \mathrm{mT}$. In order to allow a tolerance margin for practical effects, the primary magnetic field generator is a solenoid designed to 
produce at its edge a sinusoidal magnetic flux density with amplitude of $2.5 \mathrm{mT}$ at approximately $f_{o}=100 \mathrm{kHz}$ and a DC bias of $4 \mathrm{mT}$.

To achieve this goal, a solenoid with air core was built with 5 concentric layers, $5 \mathrm{~cm}$ length, $1.5 \mathrm{~cm}$ average radius, 55 turns and a wire diameter of $0.9116 \mathrm{~mm}$ (19 AWG). The effect of multiple layers can be reasonably approximated by a simple multiplier for the magnetic flux density. The wire diameter is relevant for reducing the solenoid impedance and resisting its dissipative thermal effects. The peak magnetic flux density at the edge of this solenoid is given by

$$
B_{G M R} \approx 5 \cdot 4 \pi \cdot 10^{-7} \frac{55 \cdot I_{e x c}}{2 \cdot 5 \cdot 10^{-2}} \cdot \frac{5 \cdot 10^{-2}}{\sqrt{\left(5 \cdot 10^{-2}\right)^{2}+\left(1.5 \cdot 10^{-2}\right)^{2}}} .
$$

To generate the proposed magnetic flux density, the excitation current $I_{\text {exc }}$ is given by

$$
I_{\text {exc }} \approx 1.2 \mathrm{~A}+755 \mathrm{~mA} \cdot \sin \left(2 \pi \cdot f_{o} \cdot t\right) .
$$

In order to estimate the capacity of detecting non-magnetic metallic foreign bodies with this configuration, it is necessary to determine the reduction of the magnetic flux density emitted by the solenoid with the distance, as displayed in Fig. 12.

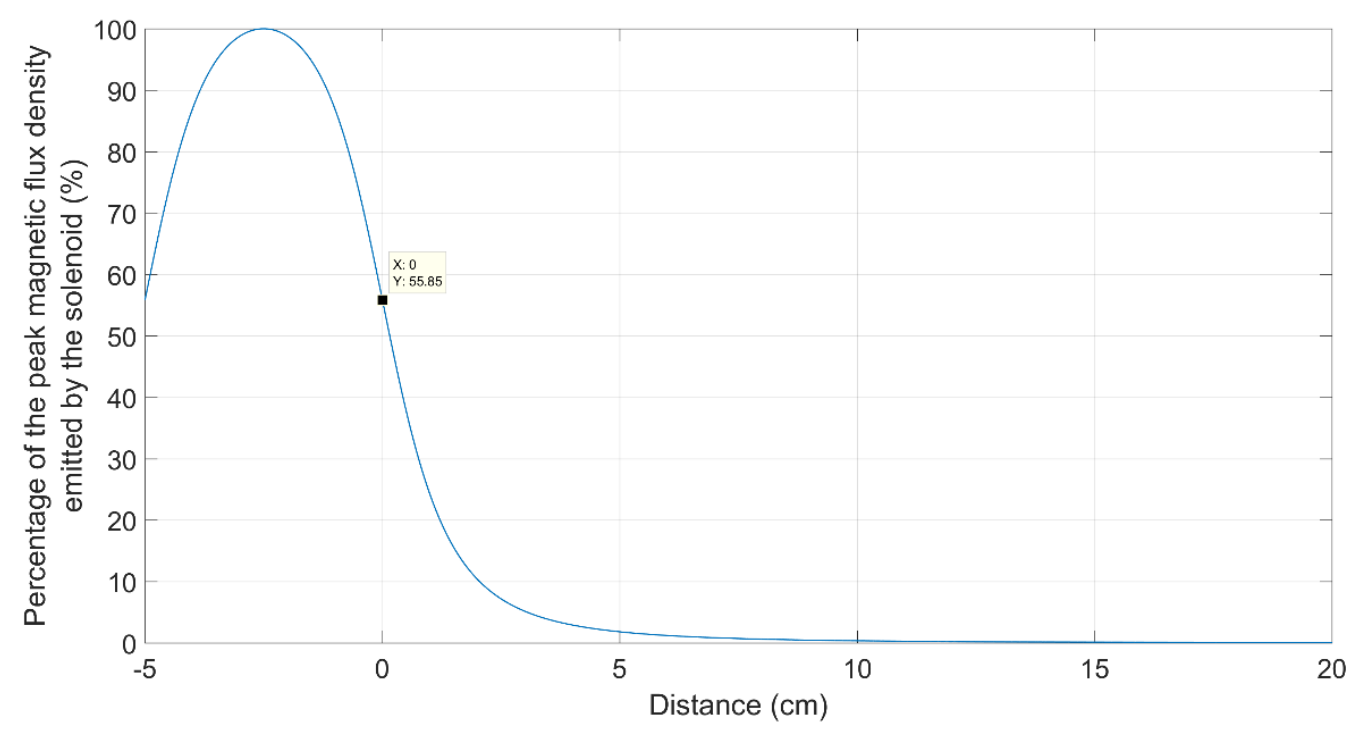

Figure 12 - Percentage of the peak magnetic flux density emitted by the solenoid with the distance, showing a high attenuation with the distance. The cursor indicates the edge of the solenoid at $D=0 \mathrm{~cm}$, where one GMR sensor is to be positioned. The other would be symmetrically located at $D=-5 \mathrm{~cm}$. 
The peak magnetic flux density occurs at the center of the solenoid and its close surroundings. Approaching the solenoid edges, the field suffers from considerable attenuation but it is outside the solenoid that the decay is most prominent.

Another challenge arises when exciting solenoids and other inductive loads at moderately high frequencies, since the impedance has a component directly proportional to the inductance times the angular frequency $(\omega \cdot L)$. When using high excitation currents, this leads to very high supply voltages.

The impedance of the solenoid was measured using a multimeter for DC values and a LRC meter for AC values. The DC impedance is purely resistive with $R_{S O L}=0.8 \Omega$, while at $f_{o}=100 \mathrm{kHz}$, the impedance can be considered as an inductance $L_{\text {sol }}=742 \mu \mathrm{H}$ in series with a resistance $R_{\text {sol }}=23.8 \Omega$. The absolute value of the complex impedance would be around $467 \Omega$. This would lead to a DC voltage of $960 \mathrm{mV}$ between the solenoid terminals superimposed with an AC voltage with amplitude of about $353 \mathrm{~V}$ at $100 \mathrm{kHz}$.

The need for high voltage and high currents power supply increases the circuit complexity, the costs and the safety risks for medical applications. This led to the design of an electronic circuit allowing the excitation of the primary magnetic field generator at high currents yet low voltages [37].

\subsection{3. \\ Primary Magnetic Field Generator Excitation}

The principle used for greatly reducing the high voltage supply is the reduction of the equivalent reactance seen by the power supply. Fig. 13 exhibits the schematics for the developed circuit. The upper section of the circuit contains the solenoid, the components for minimizing the equivalent impedance and protection components to avoid transient surges. The lower section is a controllable current drain topology, capable of producing high excitation currents. 

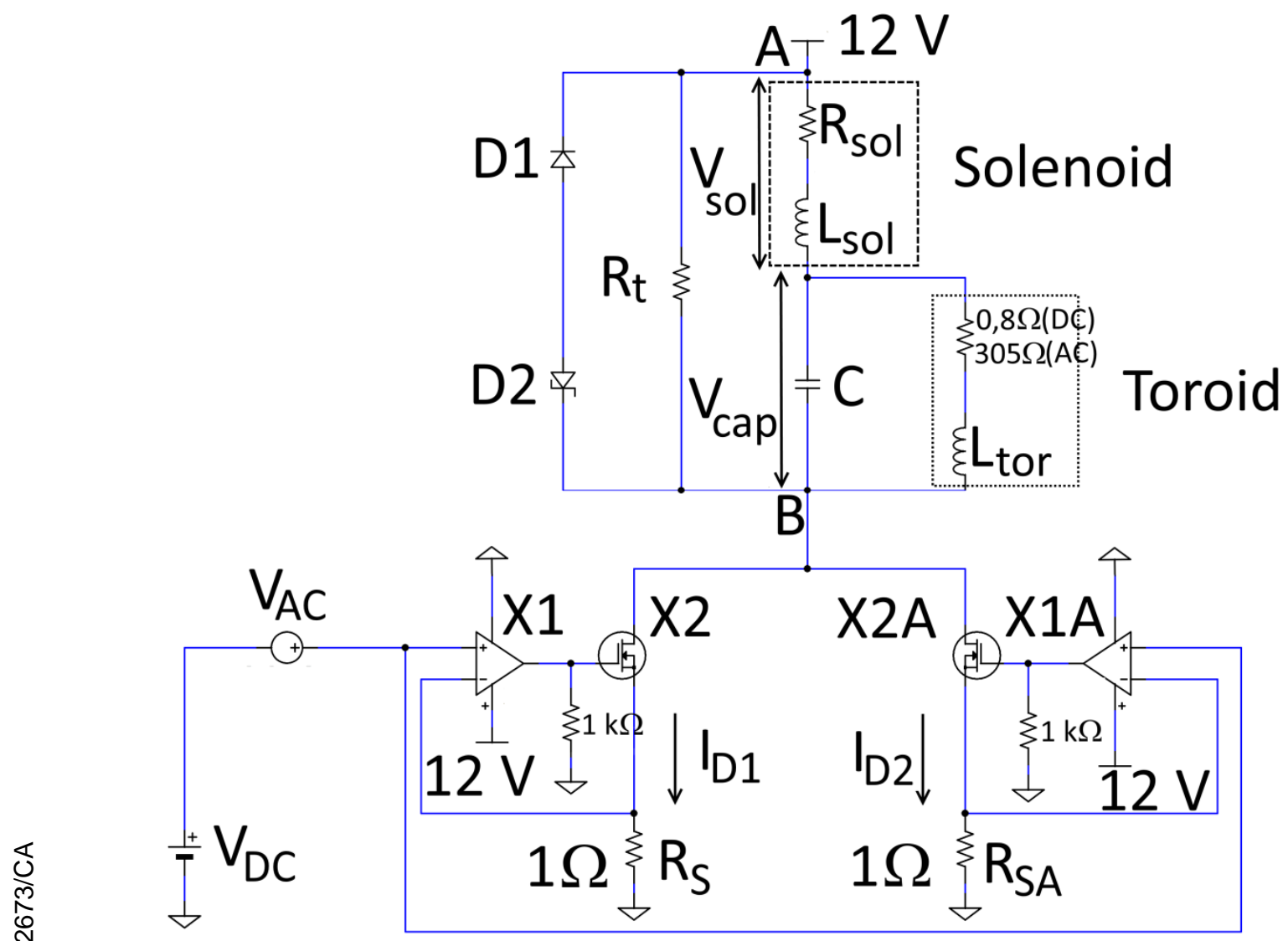

Figure 13 - Schematic for the primary magnetic field generator excitation circuit, responsible for reducing the supply voltage even for high excitation currents.

The solenoid $\left(R_{s o l}\right.$ and $\left.L_{s o l}\right)$ is placed in series with a capacitor $C$, whose value is chosen in order to make $f_{o}$ the resonance frequency. Theoretically, the equivalent impedance between the nodes $\mathrm{A}$ and $\mathrm{B}$ would tend to zero at the resonance frequency, disregarding the resistive component $R_{\text {sol }}$. The appropriate capacitance to achieve this goal can be calculated by

$$
C=\frac{1}{\left(2 \pi f_{o}\right)^{2} L_{\text {sol }}}=\frac{1}{\left(2 \pi \cdot 100 \cdot 10^{3}\right)^{2} \cdot 742 \cdot 10^{-6}} \approx 3.3 \mathrm{nF} .
$$

Since the selected GMR sensor requires a DC magnetic field bias, a DC current also must flow through the solenoid. However, this component is blocked by the capacitor $C$. A DC path is introduced by placing an inductance $L_{t o r}$ in parallel with $C$. In order to minimize its impact for the AC current path, the $L_{t o r}$ impedance needs to be much higher than the capacitor impedance at $100 \mathrm{kHz}$.

The inductance $L_{t o r}=12.1 \mathrm{mH}$ is implemented by an epoxy-coated ferrite toroid. The structure of this component concentrates most of the magnetic flux lines 
inside the closed loop. The placement of the toroid inside a metallic case shields it from high frequency electromagnetic interference. The toroid possesses a resistance of $0.8 \Omega$ at DC and $305 \Omega$ at $100 \mathrm{kHz}$.

The presence of the toroid introduces a spurious resonance frequency, according to

$$
f_{s}=\frac{1}{2 \pi \sqrt{C \cdot L_{\text {tor }}}}=\frac{1}{2 \pi \sqrt{3.3 \cdot 10^{-9} \cdot 12.1 \cdot 10^{-3}}} \approx 25.2 \mathrm{kHz}
$$

To prevent oscillations at this spurious resonance frequency, the resistance $R_{t}=$ $47 \Omega$ was placed in parallel to the previous components (solenoid, capacitor and toroid). Its value must be carefully considered, being sufficiently small to avoid instability yet not small enough to absorb the majority of the flow of the DC current, preventing its desired passing through the inductive path formed by the solenoid and the toroid.

The current passing through the complex load is controlled by a dual current source topology. It uses TL082 operational amplifiers, IRF520NS MOSFETs and $1 \Omega$ resistors ( $R_{S}$ and $R_{S A}$ ). Its current value is set by controllable DC and AC voltage sources $\left(V_{D C}\right.$ and $\left.V_{A C}\right)$, with the AC source frequency set at $f_{o}=100 \mathrm{kHz}$. A pair of identical current sources is placed in parallel to reduce the total power dissipation in each transistor. This topology could be extended by adding more current sources if even higher currents are needed.

The Zener diode $D_{2}$, specified with $10 \mathrm{~V}$ Zener voltage and capable of dissipating $15 \mathrm{~W}$ power, was added to provide surge protection when turning the circuit off, due to the nature of the inductive loads. The junction diode $D_{1}$ guarantees that the Zener diode does not affect the circuit at normal operation.

\subsubsection{1.}

\section{Simulation Results}

Computer simulations of this excitation circuit were performed in SPICE. Fig. 14 presents the voltages between the terminals of the solenoid $\left(V_{s o l}\right)$ and the capacitor $\left(V_{c a p}\right)$ as well as the current IDMOs drained by both MOSFETs (X2 and $\mathrm{X} 2 \mathrm{~A}$ ) under $V_{A C}=100 \mathrm{mV}$ and $V_{D C}=400 \mathrm{mV}$. These values were selected for experimental tests as a proof of concept, as the exact requirements for the primary magnetic field generator would need improved heat dissipation. 


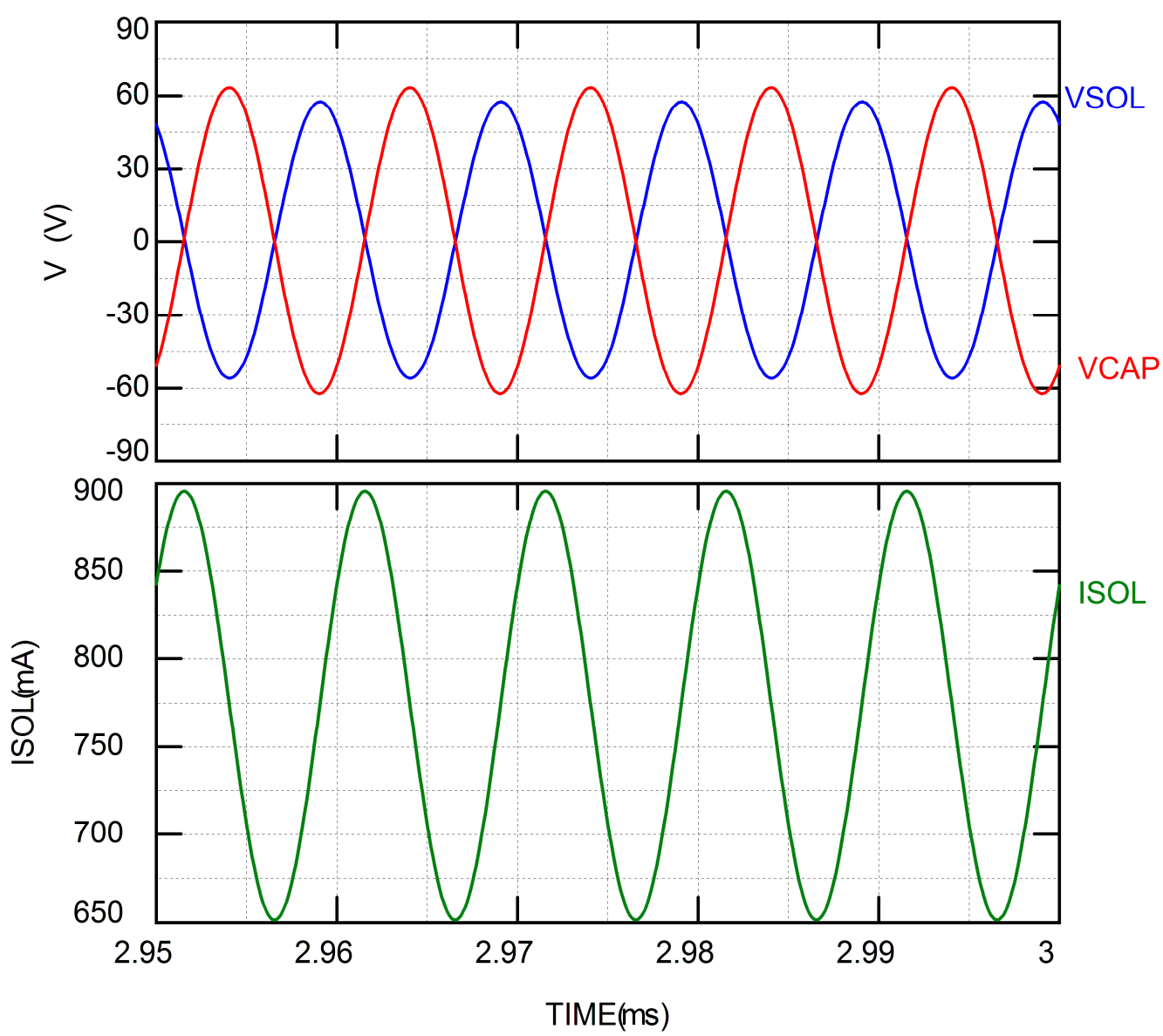

Figure 14 - Simulation results of voltage over solenoid $\left(V_{\text {sol }}\right)$, voltage over capacitor $\left(V_{\text {cap }}\right)$ and current over the solenoid ( $\left.I_{S O L}\right)$ under $V_{A C}=100 \mathrm{mV}$ and $V_{D C}=400 \mathrm{mV}$.

The simulation results confirm the expectations for the voltages $V_{\text {sol }}$ and $V_{c a p}$, which present a phase shift close to $180^{\circ}$ and similar amplitudes, coherent with a near resonance condition of phase opposition. The voltage drop between nodes $\mathrm{A}$ and B will therefore be very small, given $V_{A B}=V_{s o l}+V_{c a p}$. This is in accordance with the intended reduction of the impedance between these nodes.

The overall current flowing through the solenoid has a DC component of approximately $774 \mathrm{~mA}$ and an $\mathrm{AC}$ component at $100 \mathrm{kHz}$ with amplitude of approximately $122 \mathrm{~mA}$, levels compatible with the used excitation voltages $V_{D C}$ and $V_{A C}$ that lead to current components drained by the MOSFETs around $800 \mathrm{~mA}$ (DC) and $200 \mathrm{~mA}(\mathrm{AC})$. The differences between these values is due to the current flowing through $R_{t}$. 


\subsubsection{2.}

\section{Prototype Results}

Following the positive results of the simulations, a proof of concept prototype of the excitation circuit was implemented. Figs. 15 and 16 show the experimental results obtained when setting $V_{A C}=100 \mathrm{mV}$ at $f_{\text {exp }}=123 \mathrm{kHz}$ and $V_{D C}=400 \mathrm{mV}$.

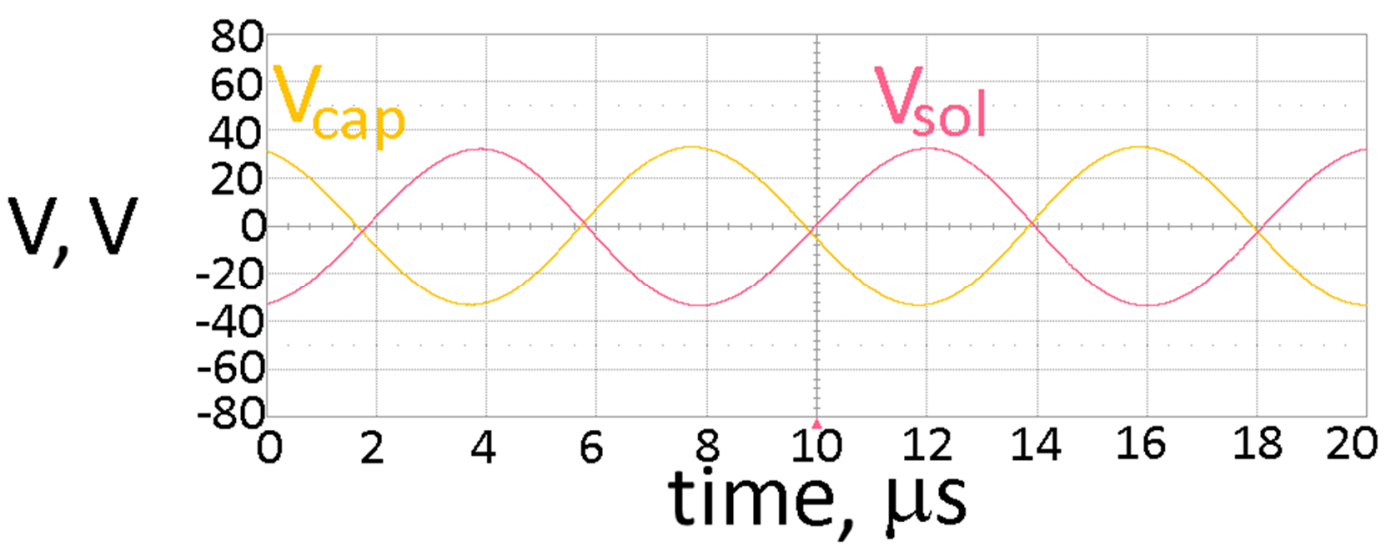

Figure 15 - Experimental values of voltages over the solenoid $\left(V_{\text {sol }}\right)$ and over the capacitor $\left(V_{\text {cap }}\right)$.

In accordance to the design and the simulation results, the voltages $V_{\text {sol }}$ and $V_{c a p}$ shown in Fig. 15 approach quadrature, however the obtained resonance frequency is $f_{\text {exp }} \approx 123 \mathrm{kHz}$ instead of the theoretical frequency $f_{o}=100 \mathrm{kHz}$. This can be explained by the non-ideal behavior of the components used, especially the inductors, since the characterization by the LCR meter uses low amplitude currents in opposition to the high currents flowing through the solenoid in the developed prototype.

In Fig. 16 are displayed the source and drain voltages from one of the MOSFETs, which theoretically are the same for both X2 and X2A. 


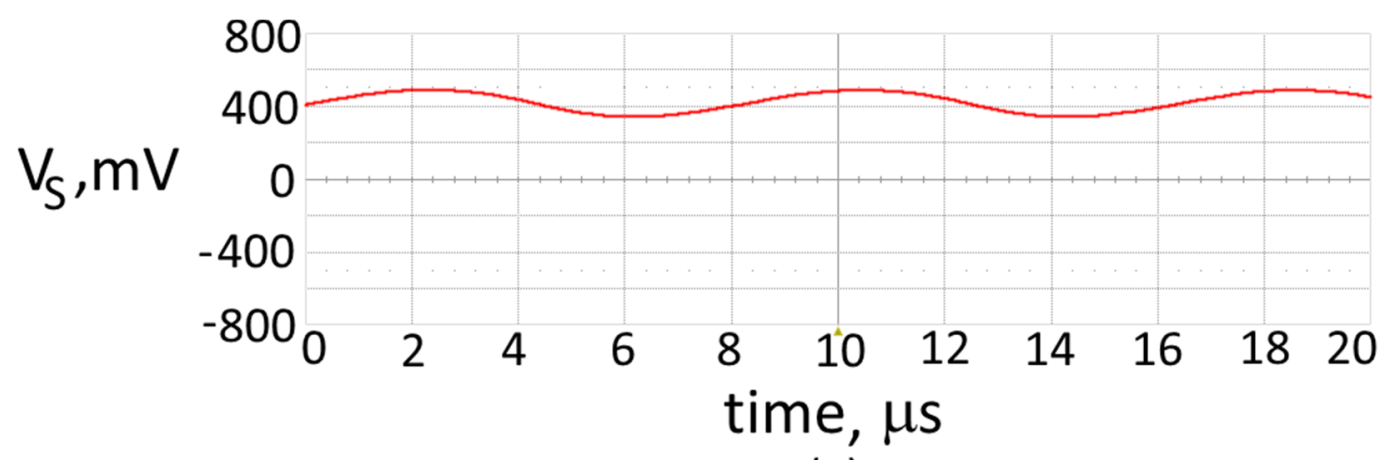

(a)

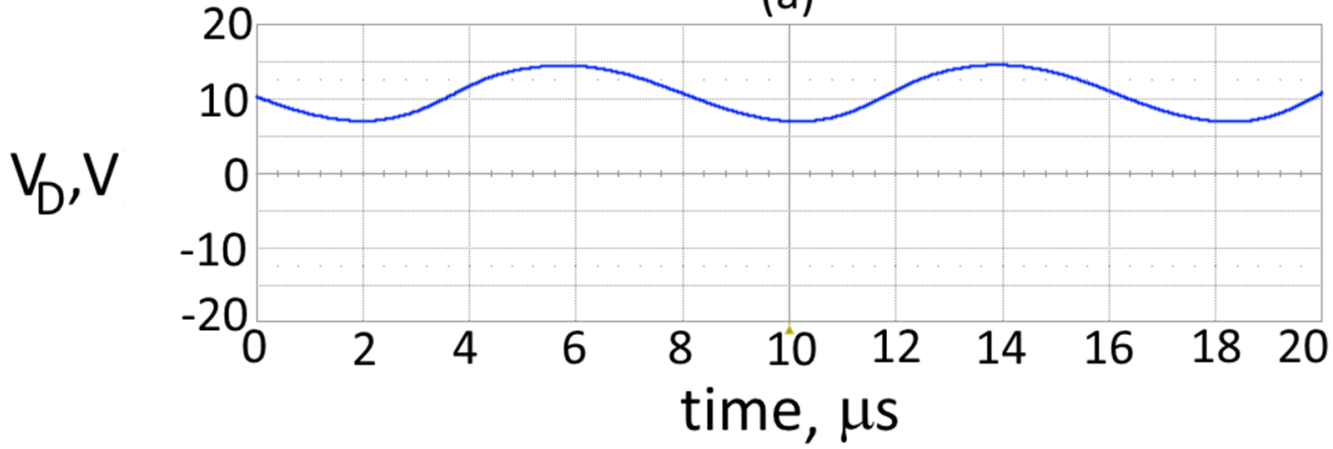

(b)

Figure 16 - Experimental values of MOSFET voltages: (a) source voltage $V_{S}$ (used to obtain the $I_{D}$ current values) and (b) drain voltage $V_{D}$.

The source voltage $V_{S}$ is directly proportional to the current $I_{D M O S}$, according to

$$
I_{D M O S}=\frac{V_{S}}{R_{S}}+\frac{V_{S A}}{R_{S A}} \approx 2 \cdot \frac{V_{S}}{R_{S}}=2 \cdot \frac{V_{S}}{1}=2 V_{S} .
$$

Thus, the obtained experimental value for the current $I_{D M O S}$ is

$$
I_{D M O S}=800 \mathrm{~mA}+200 \mathrm{~mA} \cdot \sin (2 \pi \cdot 123 \mathrm{kHz} \cdot t) .
$$

Regarding the drain voltage $V_{D}$, it indicates the overall voltage drop between nodes $\mathrm{A}$ and $\mathrm{B}$, since it can be obtained from $V_{A B}=12 \mathrm{~V}-V_{D}$. The mean value of $V_{D}$ is about $10.3 \mathrm{~V}$, indicating a DC voltage drop of approximately $1.7 \mathrm{~V}$ at $V_{A B}$. The amplitude of the AC component of $V_{D}$ is also small at about $3.7 \mathrm{~V}$.

These results prove the solenoid excitation circuit is effective at creating both DC and AC low voltage paths for high currents. This circuit could be easily adapted for higher currents, in order to match the primary magnetic flux density values required for the location of non-magnetic metallic foreign bodies.

\subsection{4.}

\section{Signal Processing Circuit for Gradiometric Reading}

The signal processing circuit is responsible for filtering and amplifying the signals obtained from the sensor elements and performing the gradiometric reading 
through a final differential stage. The instrumentation amplifier INA129 used has many favorable characteristics, as explained in Appendix A. The schematic for the proposed circuit is shown in Fig. 17.

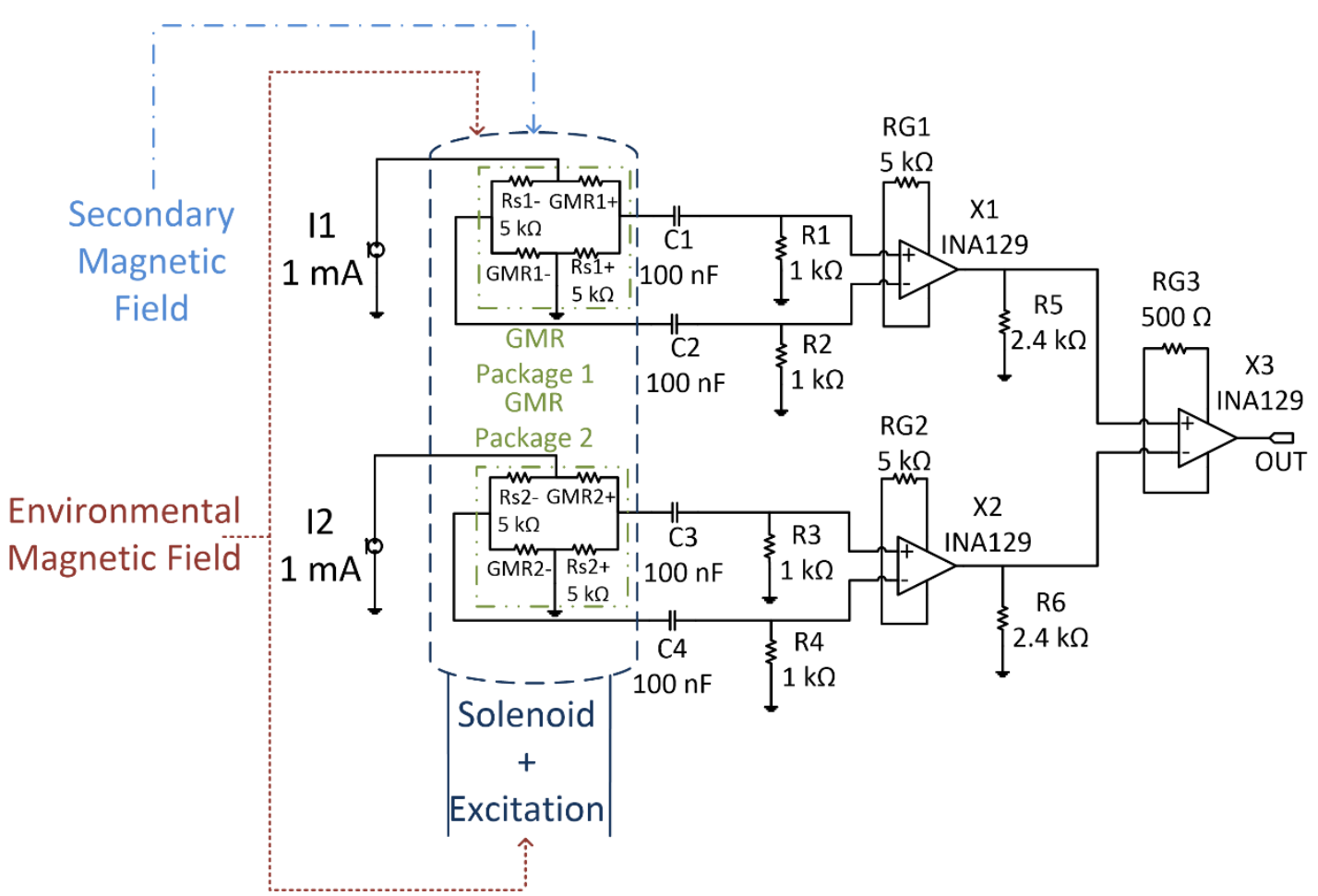

Figure 17 - Simplified schematic for the signal processing circuit including the GMR sensor packages, showing their internal bridge composition.

The bridge internal configuration of each GMR is already a first differential stage, ensuring more reliable measurements. Each GMR half bridge output is connected to high pass filters, removing the DC bias and reducing the pink noise $(1 / f)$, typically predominant in magnetic sensor elements. The filter outputs are connected to the inputs of an instrumentation amplifier. This performs a differential reading of each GMR sensor output, also amplifying the signals, serving as the intermediate processing stage of Fig. 11. The final differential stage amplifies and subtracts the signals originating from both GMR sensors, effectively producing a gradiometric reading.

The GMR packages are supplied by DC current sources of $1 \mathrm{~mA}$, leading to a sensitivity of $22.5 \mathrm{~V} / \mathrm{T}$. The instrumentation amplifiers have voltage supplies of $\pm 18 \mathrm{~V}$. 
The resistors RG1, RG2 and RG3 are used to adjust the gains of the instrumentation amplifiers. When using INA129 amplifiers, making RG1 = RG2 = $5000 \Omega$ leads to a theoretical gain of $10.88 \mathrm{~V} / \mathrm{V}$ for the first instrumentation amplifiers. Using RG3 $=500 \Omega$ leads to a theoretical gain of $99.8 \mathrm{~V} / \mathrm{V}$. This is the maximum gain that maintains an amplifier bandwidth of $100 \mathrm{kHz}$. The final theoretical gain would be $1085.824 \mathrm{~V} / \mathrm{V}$. Simulations in TopSpice indicated this is the optimal gain configuration, since other settings either produced noticeable signal distortion or had insufficient output signal levels. Similarly, the resistors R5 and R6 of $2.4 \mathrm{k} \Omega$ were placed to avoid signal distortions found in the simulations.

\subsubsection{1.}

\section{High Pass Filters and New Estimated Resolution}

Each GMR output passes through a simple first order high pass RC filter with cutoff frequency of about $1591 \mathrm{~Hz}$. The main purpose is to remove or greatly attenuate the DC component of the signals since they would saturate the instrumentation amplifier stages. Another greatly desirable characteristic is attenuating the predominant $1 / \mathrm{f}$ noise in the GMR outputs. This allows a reasonable new estimate for the best resolution possible.

Using the noise spectral power density found at eq. (79) and integrating over the frequency bandwidth leads to the rms value for the noise due to the GMR sensor. This is shown in eq. (89), where the frequency goes from $f l=1591 \mathrm{~Hz}$ to the cutoff frequency of the instrumentation amplifiers at $100 \mathrm{~V} / \mathrm{V}$ gain, which is close to $f 2=100 \mathrm{kHz}$. The Equivalent Noise Bandwidth factor of 1.57 is used for both cutoff frequencies. The sensibility Sens is used to convert the noise voltage into a magnetic flux density value.

$$
\text { NoiseGMR } R_{r m s}=\frac{1}{\operatorname{Sens}} \sqrt{\int_{1.57 \cdot f 1}^{1.57 \cdot f 2} \frac{s_{\text {onoise }}}{f} d f}=\frac{1}{22.5} \sqrt{\int_{1.57 \cdot 1591}^{1.57 \cdot 10^{5}} \frac{1.05 \cdot 10^{-12}}{f} d f}=92.5 \mathrm{nT}
$$

This noise would give an rms voltage of $v_{n G M R}=2.08 \mu \mathrm{V}$, which would be amplified by each instrumentation amplifier stage. Therefore, the final output noise would have a rms voltage of $2.08 \mathrm{mV}$.

Once again using the factor 6.6 to convert from rms value to peak-to-peak with $99.9 \%$ confidence, the expected peak-to-peak noise due to the sensor is

$$
\text { NoiseGMR }_{p p}=6.6 \cdot \text { NoiseGMR } R_{r m s}=610 \mathrm{nT} \approx 0.6 \mu \mathrm{T} \text {. }
$$


This is a good ballpark estimate for the lowest possible noise from the sensor and, therefore, the best possible resolution at this bandwidth when ignoring other sources of noise. However, the bandwidth used is reasonably large at about $98409 \mathrm{~Hz}$, which means other noise sources could be at similar voltage levels. Since the gain of each amplification stage is over $10 \mathrm{~V} / \mathrm{V}$ the most significant noise will be at the first instrumentation amplifier inputs.

The noise introduced at the input of each instrumentation amplifier (in-amp) can also be estimated from the noise voltage density curves presented in its datasheet. At these frequencies, the in-amp noise is predominantly white. For a gain of $10 \mathrm{~V} / \mathrm{V}$ and the utilized bandwidth $B$ (adjusted by the Equivalent Noise Bandwidth factor), a good estimate of the noise voltage is

$$
v_{n A m p}=\sqrt{\left(14 \cdot 10^{-9} \mathrm{~V} / \sqrt{\mathrm{Hz}}\right)^{2} \cdot 1.57 \cdot(100 \mathrm{kHz}-1591 \mathrm{~Hz})}=5.50 \mu \mathrm{V} .
$$

This value is about 2.5 times higher than the GMR noise, therefore it is more significant. Since they are still at about the same order, a good estimate will have to consider both.

The last considerable source of noise in this circuit would be the thermal noise. Each $1 \mathrm{k} \Omega$ resistor thermal noise voltage is given by eq. (92), where $k_{B}$ is the Boltzmann constant, $T$ is the temperature in Kelvin, $R$ is the resistance and $B$ is the bandwidth adjusted by the Equivalent Noise Bandwidth factor.

$$
v_{n}=\sqrt{4 k_{B} T R B}=\sqrt{4 \cdot 1.38 \cdot 10^{-23} \mathrm{~J} / \mathrm{K} \cdot 298.15 \mathrm{~K} \cdot 1000 \Omega \cdot 1.57 \cdot(98409 \mathrm{~Hz})}=1.59 \mu \mathrm{V}
$$

This noise voltage is very close to the GMR output noise and does affect the final resolution estimate, especially because there is a resistor at each instrumentation amplifier input. The total noise voltage at the instrumentation amplifier inputs would follow

$$
v_{\text {noise eq }}=\sqrt{\left(v_{n G M R}\right)^{2}+\left(v_{n A m p}\right)^{2}+2 \cdot\left(v_{n}\right)^{2}}=\sqrt{\left(2.08 \cdot 10^{-6}\right)^{2}+\left(5.50 \cdot 10^{-6}\right)^{2}+2 \cdot\left(1.59 \cdot 10^{-6}\right)^{2}}=6.30 \mu \mathrm{V} .
$$

The equivalent magnetic flux density noise in peak-to-peak with $99.9 \%$ confidence is given by

$$
\text { noise }_{p p}=6.6 \cdot \frac{v_{\text {noise eq }}}{\text { Sens }}=6.6 \cdot \frac{6.30 \cdot 10^{-6}}{22.5}=1.85 \mu \mathrm{T} \approx 2 \mu \mathrm{T} .
$$

Therefore, for this circuit the expected resolution would be $2 \mu \mathrm{T}$. Considering the larger component of this noise voltage is the instrumentation amplifier white noise, even reducing the bandwidth by using more selective filters would have small 
impact over this value while greatly increasing circuit complexity and component number. The speed of the signal processing would also be dramatically reduced.

One simple possibility for noise reduction is changing the excitation frequency from $100 \mathrm{kHz}$ to a value under $10 \mathrm{kHz}$. That would make using a narrower bandwidth of about $2 \mathrm{kHz}$ less complex, which would reduce all the noise components by a factor of nearly 50, resulting in a resolution close to 10 times smaller as well (about $300 \mathrm{nT}$ ). The main tradeoff would be the considerable attenuation of the secondary magnetic fields that benefit from frequencies close to $100 \mathrm{kHz}$.

The most effective option would be the use of a lock-in amplifier at the circuit output. This instrument can potentially narrow the bandwidth to even under $1 \mathrm{~Hz}$ and present both amplitude and phase of the signal of interest. By using a $1 \mathrm{~Hz}$ bandwidth value centered in $100 \mathrm{kHz}$ in eqs ( 89 - 94), a resolution as low as $5.6 \mathrm{nT}$ would be theoretically possible. The tradeoffs would be cost, portability and also processing speed.

\subsubsection{2.}

\section{Estimated Minimum Detectable Diameter}

Considering the designed circuit conditions with the GMR estimated resolution of $2 \mu \mathrm{T}$ and using the equations provided in Chapter 3 , the secondary magnetic flux density values can be estimated for varying diameters and distances. Since each GMR sensor package is very small, the base-line is approximately the length of the solenoid $(5 \mathrm{~cm})$. Fig. 18 shows some of these results. 


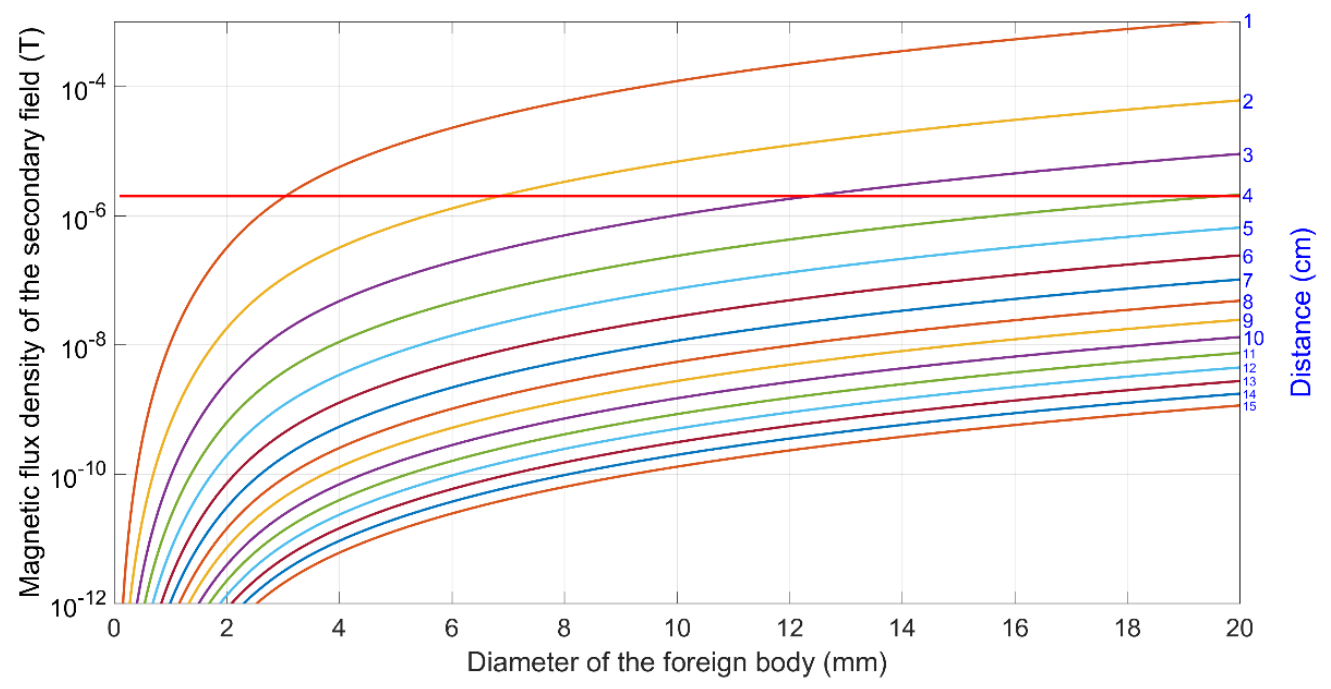

Figure 18 - Curves for the peak secondary magnetic flux density $\left(B_{\operatorname{smax}}\right)$ (in $\mathrm{T}$ ) varying with the diameter of the foreign body (in $\mathrm{mm}$ ), for a given distance (in $\mathrm{cm}$ ). Each curve's number at right represents the distance in $\mathrm{cm}$ between the projectile and the detector in that case. The red line represents the estimated resolution of the GMR detection system at $2 \mu \mathrm{T}$.

Each curve represents the peak magnetic flux density generated by the eddy currents varying with the diameter of the foreign body at a fixed distance. The red line stands for the originally estimated resolution of the GMR system $(2 \mu \mathrm{T})$. Therefore, magnetic flux values above this red line would be theoretically detectable by the sensor element. This leads to Fig. 19, displaying the minimum detectable diameter for this sensor element varying with the distance.

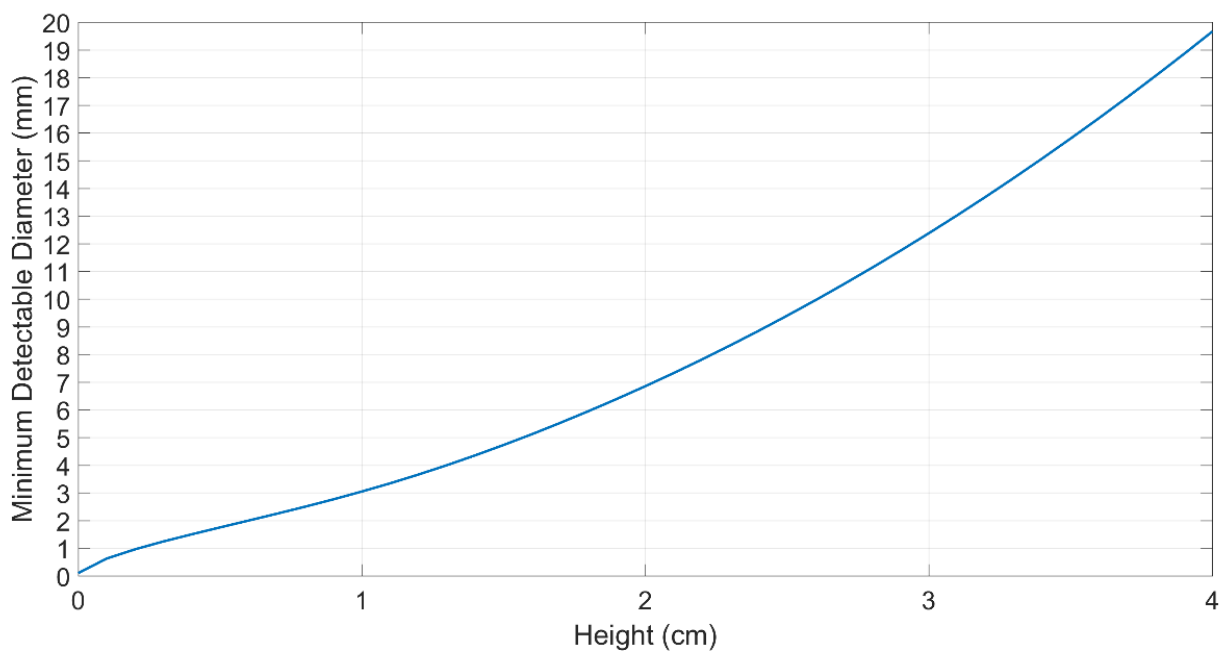

Figure 19 - Minimum detectable diameter of the non-magnetic metallic foreign body varying with the distance from the GMR location system. 
These preliminary results indicate the GMR location system can detect foreign bodies at close ranges only. A typical $9 \mathrm{~mm}$ diameter projectile can be detected for distances up to $2.4 \mathrm{~cm}$. Smaller shrapnel would be detectable only closer to $1 \mathrm{~cm}$ distances. The GMR sensor is well below a SQUID performance. Considering the great difference between the two sensor elements in sensitivity and resolution, this is still a good result, showing that low cost alternatives can be explored.

\subsubsection{3.}

\section{Instrumentation Amplifier Considerations}

The GMR sensors output is expected to be around $146.25 \mathrm{mV}$ for the utilized operation point. The first amplifier stage gain is $10.88 \mathrm{~V} / \mathrm{V}$ with an expected CMRR around $50 \mathrm{~dB}$. The second stage gain is $99.8 \mathrm{~V} / \mathrm{V}$ and its CMRR is about $76 \mathrm{~dB}$. The predicted maximum signal of interest $V_{\text {dmax } 2}$ before saturation of the second stage is given by

$$
\begin{gathered}
V_{\text {max } 2}=17.15 \approx 99.8\left(V_{d \max 2}\right)+0.0158 \frac{\left(2 \cdot 146.25 \cdot 10^{-3}+V_{d \max 2}\right)}{2} \\
V_{d \max 2} \approx \frac{17.15-0.0231}{99.8+0.0079}=171.8 \mathrm{mV} .
\end{gathered}
$$

Similarly, the predicted maximum signal of interest $V_{\text {dmax } 1}$ before saturation of the first stage is

$$
\begin{gathered}
V_{\text {max } 1}=17.15 \approx 10.88\left(V_{d \max 1}\right)+0.0344 \frac{\left(146.25 \cdot 10^{-3}+V_{d \max 1}\right)}{2} \\
V_{d \max 1} \approx \frac{17.15-0.0503}{100+0.00172}=1.57 \mathrm{~V} .
\end{gathered}
$$

The second stage saturates for a secondary magnetic flux density of approximately $702 \mu \mathrm{T}$ while the first stage accepts signals up to $69.9 \mathrm{mT}$. The first stage output limitation is beyond the linear operation range of the GMR sensor but the second stage is not. However, since Fig. 18 shows the expected secondary magnetic fields are typically under $500 \mu \mathrm{T}$, this upper limit does not have a great influence in the detection system performance even though it diminishes the linear operation range of the circuit.

Since the cascading of instrumentation amplifiers can enhance the effects of non-ideal and non-linear aspects of these components, it is important to note these estimated values can be different from the actual experimental results. 


\subsubsection{4.}

\section{Simulation Results}

Simulated results for this topology are shown in Fig. 20.

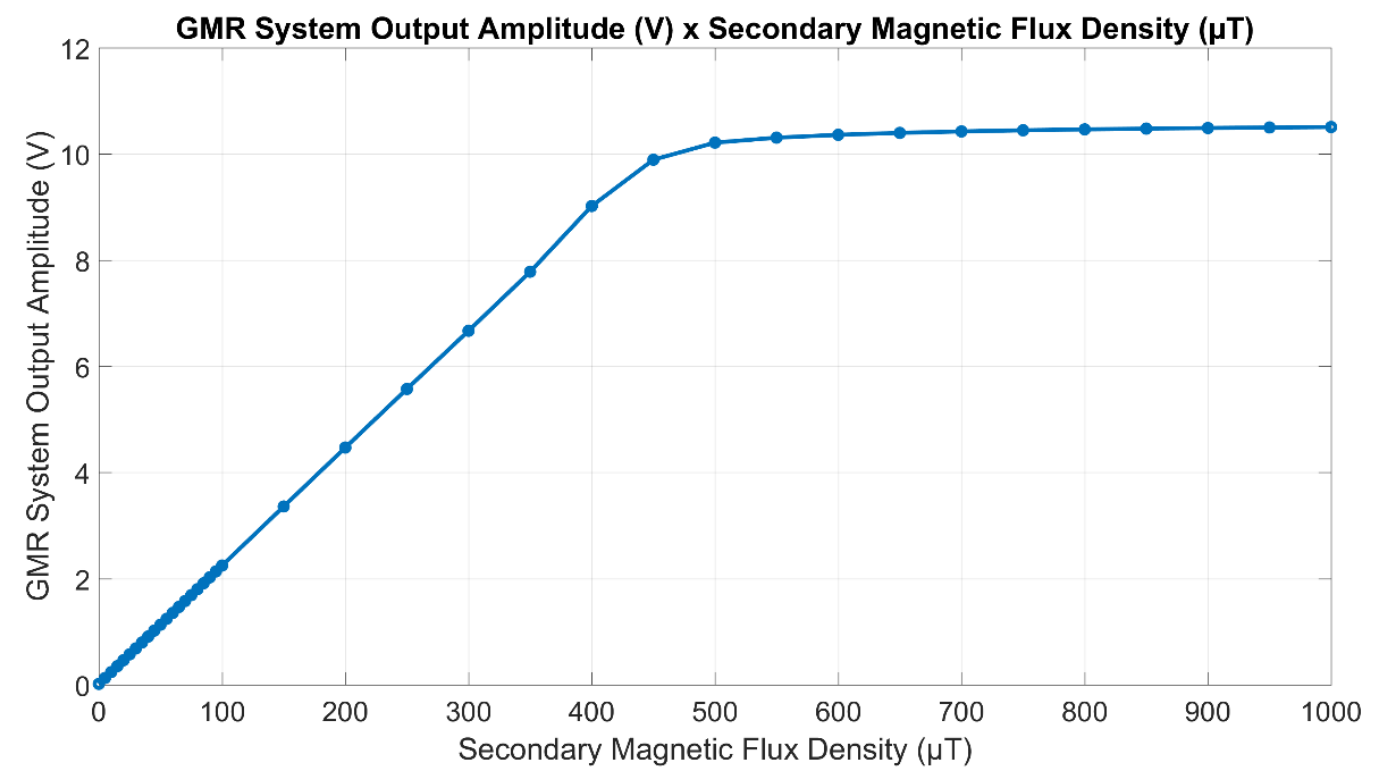

Figure 20 - Simulated results for output voltage amplitude (V) versus secondary magnetic flux density $(\mu T)$ on the GMR signal processing circuit for gradiometric reading. Samples between $100 \mu \mathrm{T}$ to $1000 \mu \mathrm{T}$ were obtained in $50 \mu \mathrm{T}$ steps.

The complete system simulation results show a linear region for secondary magnetic flux densities ranging from 0 to approximately $400 \mu \mathrm{T}$, after which the output is distorted and saturates. This limitation is lower than what was estimated in the previous section. Fig. 21 shows only the linear region. 


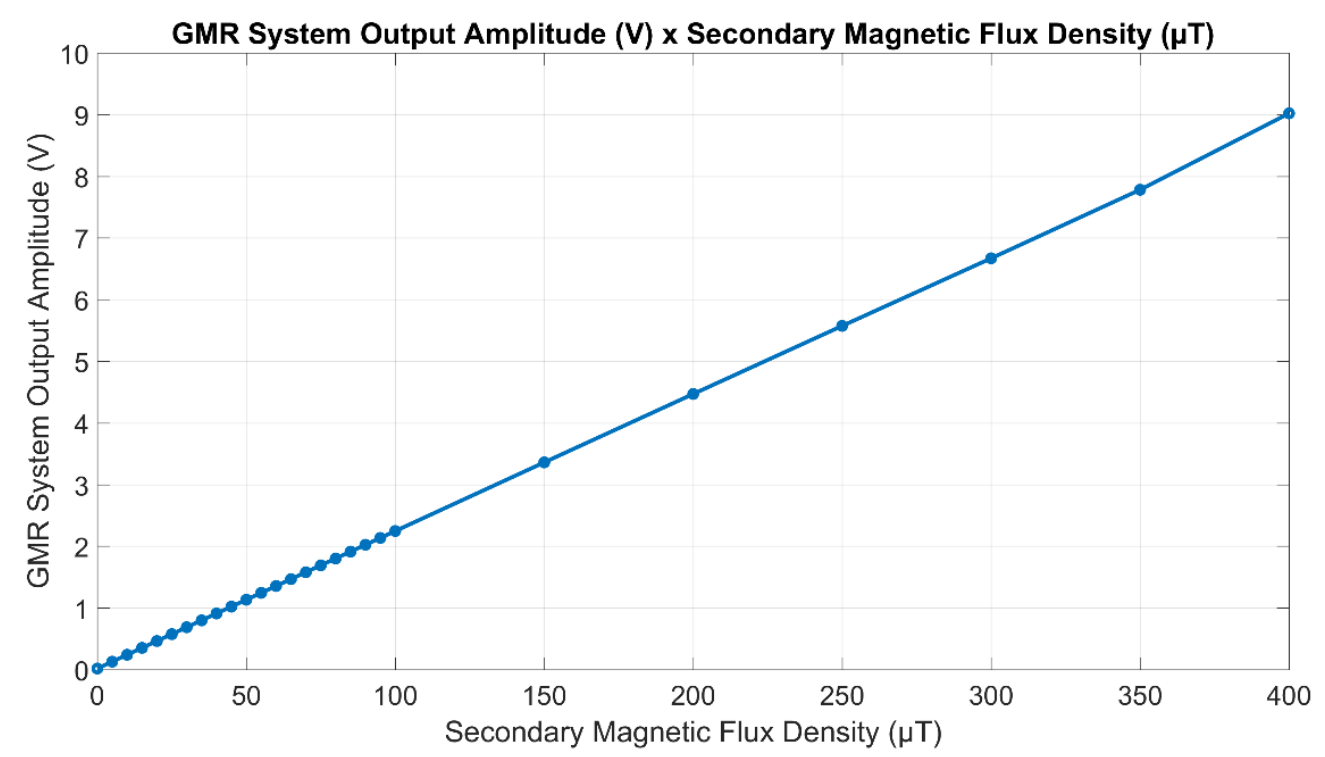

Figure 21 - Region considered linear for the output voltage amplitude (V) versus secondary magnetic flux density $(\mu T)$ on the GMR signal processing circuit for gradiometric reading. This ranges from 0 to approximately $400 \mu \mathrm{T}$.

Applying a trend line results in a sensitivity around $22300 \mathrm{~V} / \mathrm{T}$ in the linear region. The original sensitivity for each GMR sensor under the supply conditions used (current of $1 \mathrm{~mA}$ ) is $22.5 \mathrm{~V} / \mathrm{T}$, so this suggests an effective gain of $991.11 \mathrm{~V} / \mathrm{V}$ for the circuit, about $9 \%$ less than the theoretical gain of $1085.824 \mathrm{~V} / \mathrm{V}$. Non-ideal aspects of the instrumentation amplifiers are responsible for this result, including the Gain-Bandwidth Product (GBW) and the lower Common-Mode Rejection Ratio (CMRR) at $100 \mathrm{kHz}$ (around $50 \mathrm{~dB}$ for gain $10 \mathrm{~V} / \mathrm{V}$ and around $70 \mathrm{~dB}$ for gain $100 \mathrm{~V} / \mathrm{V})$. Fig. 22 shows a range closer to the GMR sensor estimated resolution. 


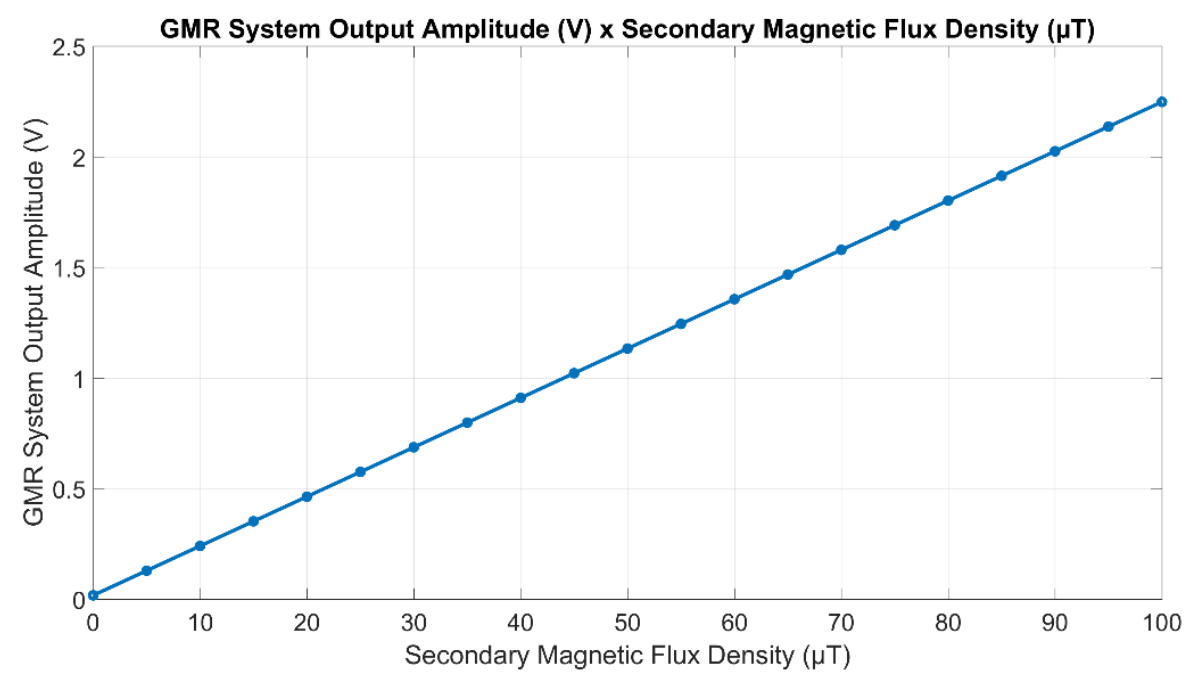

Figure 22 - Output voltage amplitude (V) of the GMR system for secondary magnetic flux densities $(\mu \mathrm{T})$ between 0 to $100 \mu \mathrm{T}$ in $5 \mu \mathrm{T}$ steps (close to the estimated resolution of the sensor at $2 \mu \mathrm{T}$ ).

Although still hard to observe visually, there is an offset amplitude of $17.43 \mathrm{mV}$ present in the absence of a secondary magnetic flux density. This is heavily influenced by the CMRR of the instrumentation amplifier. Under previous studies [36], these effects led to non-linearity for fields under $200 \mathrm{nT}$ and impacted the overall output gain but finer adjustments of circuit components managed to resolve those issues, as seen here where the output continues to be linear despite the amplitude offset.

In order to compare the complete system and the original GMR sensor a logarithmic scale plot of the output amplitude curve in function of the secondary magnetic flux density can be used, as shown in Fig. 23. The offset present in the complete system output amplitude is removed to preserve the linearity of the curve for better comparison. The GMR output is considering the selected operating point of a $40 \mathrm{Oe}=4 \mathrm{mT}$ DC primary magnetic flux density. 


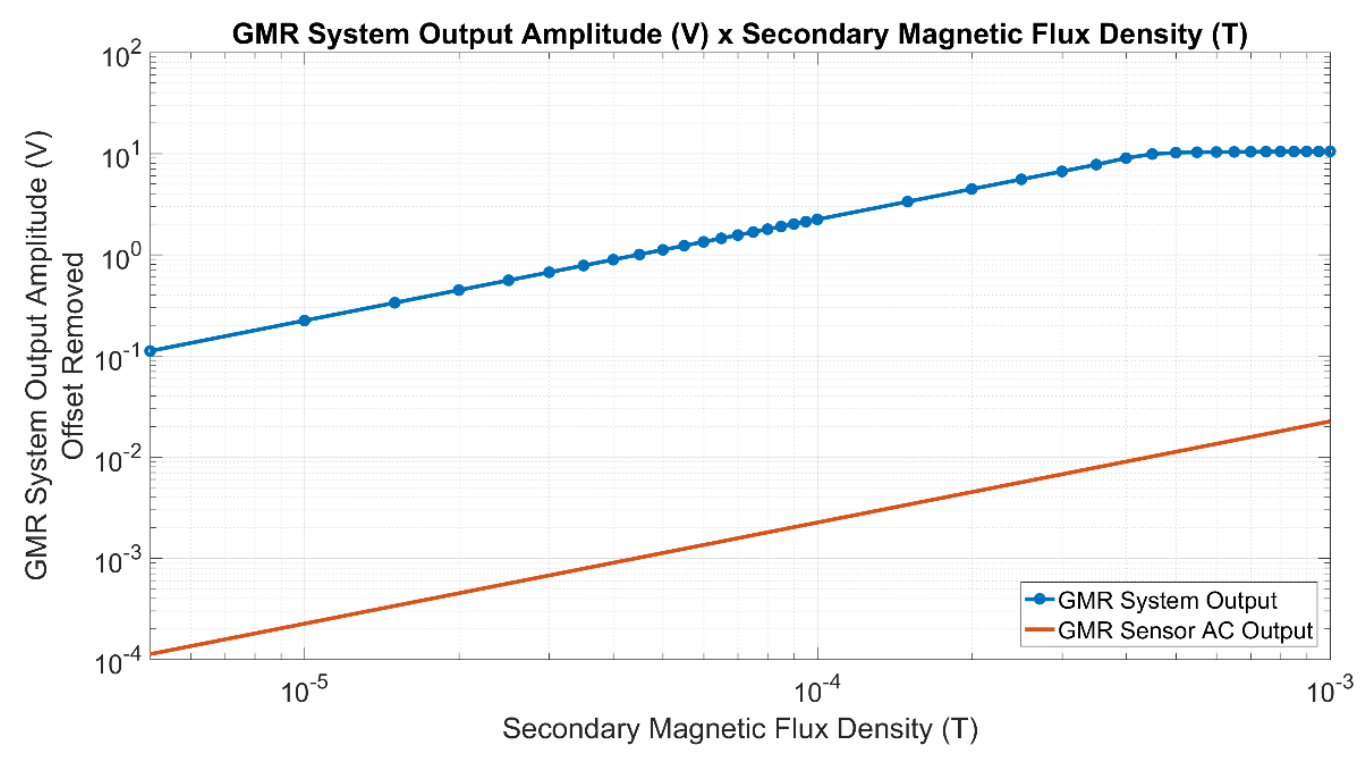

Figure 23 - Logarithmic plot comparison of output amplitudes (V) between an individual GMR sensor and the complete detection system versus the secondary magnetic flux density (mT). The GMR curve is valid for the operating point of $40 \mathrm{Oe}=4 \mathrm{mT}$ DC primary flux density. The system output has the amplitude offset of $\mathbf{1 7 . 4 3} \mathbf{~ m V}$ subtracted.

This operating point guarantees the GMR sensor possesses a linear output range for AC magnetic field amplitudes starting from the estimated resolution $(20 \mathrm{mOe}=2 \mu \mathrm{T})$ up to $30 \mathrm{Oe}=3 \mathrm{mT}$. The processing circuit limits the linear output range to a maximum of $400 \mu \mathrm{T}$ as a tradeoff for introducing a considerable gain. For fields up to $400 \mu \mathrm{T}$, the output from the GMR sensor goes from $112.5 \mu \mathrm{V}$ to $9.0 \mathrm{mV}$ while the system output goes from $111.5 \mathrm{mV}$ to $9.0 \mathrm{~V}$. This can lead to more accurate measurements by reducing the impact from electronic noise and making readings more reliable. Fig. 24 presents a point-by-point gain curve introduced by the processing circuit over the GMR output in the detecting system linear range. 


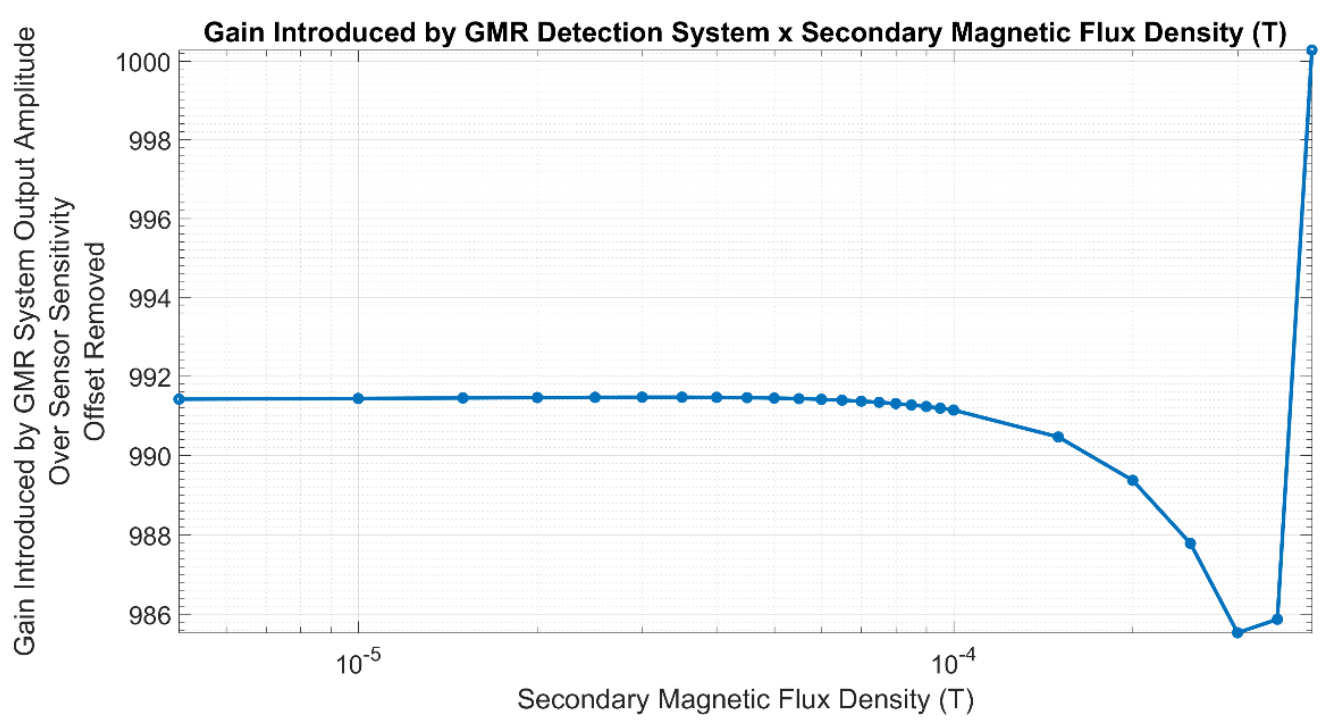

Figure 24 - Gain introduced by the signal processing circuit over the GMR sensor in V/V calculated for each simulated point for secondary magnetic fields ranging from $5 \mu \mathrm{T}$ to $400 \mu \mathrm{T}$.

For the better part of this range the gain is stable at approximately $991.4 \mathrm{~V} / \mathrm{V}$. The calculated gains show that a more strictly linear region would be under $100 \mu \mathrm{T}$ since there is already a noticeable variation above this value. However, the gain variation is still under $1 \%$, possibly allowing the assumption of linearity between $100 \mu \mathrm{T}$ and $400 \mu \mathrm{T}$ as long as these measurements are stated as slightly less reliable.

The greater challenge for the GMR based foreign body locator is the dissociation between the primary and the secondary magnetic field signals, since the first is much higher than the latter. A higher gain in the instrumentation amplifiers improves common-mode rejection and increases the voltages due to the secondary field, improving the gradiometric reading. However, the relatively high voltage due to the primary magnetic flux density can lead to output saturation for higher gains. Balancing these conditions proves difficult and can lead to the nonlinearity present when the common-mode output of the final instrumentation amplifier is at similar levels to the desired signal.

Before an experimental test with a prototype for the signal processing circuit could be realized, a new GMI sensor element prototype was made commercially available that presents desirable characteristics including much higher sensitivity and no need for a DC magnetic field bias, having the potential of great improvement over the main challenges present with the GMR projects. This led to the adaptation 
of the developed technique for this new sensor element, as described in the following section.

\section{2. \\ Developments Based on GMI Sensors}

The Aichi Multi-type Nano-tesla Sensor (MI-CB-1DJ-M-B) operates at lower frequencies than the GMR sensor NVE AA-005-002, being limited to a maximum of $10 \mathrm{kHz}$. This characteristic, associated to the lack of a DC magnetic field bias requirement greatly simplifies the solenoid excitation, since these were the factors that led to high solenoid impedances and, consequently, high terminal voltages. Therefore, a simple setup of a signal generator and a known resistor in series with the solenoid can be used. The main limiting factor is the generator output current, so a new solenoid was designed accordingly.

\subsection{1. \\ Model used: Aichi Multi-type Nano-tesla Sensor (MI-CB-1DJ-M-B)}

The GMI sensor prototype chosen possesses a typical sensitivity of $5.0 \mathrm{~V} / \mu \mathrm{T}$, approximately 32000 times higher than the maximum sensitivity for the GMR AA005-02 when using a voltage supply of $24 \mathrm{~V}$. This higher sensitivity however is more limited in the linear operation range and bandwidth. The GMI sensor is supposed to measure magnitudes for magnetic flux densities under $1.0 \mu \mathrm{T}$ or $10^{-2}$ Oe and its operating bandwidth is between $0.1 \mathrm{~Hz}$ and $10 \mathrm{kHz}$.

Each GMI sensor has a large size compared to the compact SOIC8 package of the GMR because they are embedded in a small circuit board, including voltage references, DC blocking filters and internal amplification. Fig. 25 is a simplified diagram showing the main dimensions of the MI-CB-1DJ-M-B sensor. 


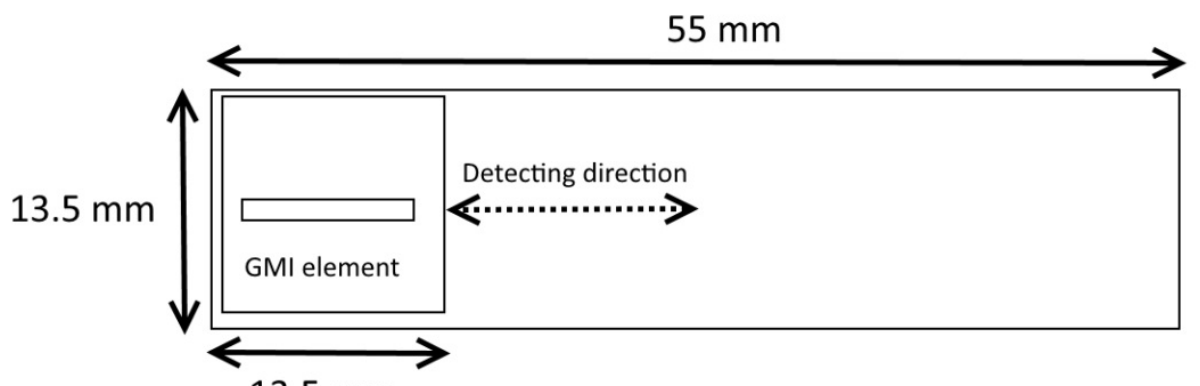

$13.5 \mathrm{~mm}$

Figure 25 - Simplified diagram with dimensions of the GMI sensor prototype Aichi MI-CB-1DJ-M-B.

Aichi sells two versions of each sensor, regarding the number of sensor elements to be used: single-type and multi-type. The former includes an onboard oscillator to excite the sensor element, while the latter requires the use of an external oscillator to synchronize two or more sensors. The oscillators are specified as square-waves with $50 \%$ duty cycle and voltage range from $0 \mathrm{~V}$ to $5 \mathrm{~V}$ at the frequency of $1 \mathrm{MHz}$. In order to allow the implementation of a first order gradiometer, the multi-type version was selected.

Aichi also manufactures sensors with one of two detecting directions: A (perpendicular to the length of the circuit board) and B (parallel to the length of the circuit board). Direction B was favored as it is more appropriate for $\mathrm{z}$ axis measurements.

The typical noise presented (between $0.3 \mathrm{nT}$ to $1.0 \mathrm{nT}$ in peak-to-peak values and with noise density of $10 \mathrm{pT} / \sqrt{\mathrm{Hz}}$ ) is already smaller than the GMR estimated noise level. Since the major noise component is $1 / \mathrm{f}$ (pink noise), the use of appropriate filters for the passing of only selected higher frequencies can greatly diminish the noise level and lead to better resolution for the foreign body locating system.

Overall, the benefits of higher sensitivity and lower noise levels compensate for the other limitations of this sensor element, making the use of this GMI sensor a great improvement for the objective of a non-ferromagnetic metallic foreign body locating system.

Table 4 is a summary of the main characteristics of the selected GMI sensor prototype. 
Table 4 - Main characteristics for GMI sensor Aichi MI-CB-1DJ-M-B.

\begin{tabular}{|c|c|c|c|c|}
\hline GMI characteristics & Minimum & Typical & Maximum & Unit \\
\hline \multirow{2}{*}{$\begin{array}{c}\text { Linear Range } \\
\text { (in air, with DC field under } \pm 30 \\
\mu \mathrm{T} \text { ) }\end{array}$} & -10 & & 10 & $\mathrm{mOe}$ \\
\hline & -1 & & 1 & $\mu \mathrm{T}$ \\
\hline \multirow{2}{*}{$\begin{array}{l}\text { Sensitivity } \\
\text { (in air, at } 10 \mathrm{~Hz} \text { ) }\end{array}$} & 0.45 & 0.5 & 0.55 & $\mathrm{~V} /(\mathrm{mOe})$ \\
\hline & 4.5 & 5.0 & 5.5 & $\mathrm{~V} /(\mu \mathrm{T})$ \\
\hline Output Saturation Voltage & & 14.9 & & V \\
\hline $\begin{array}{c}\text { Normal Output Voltage (at } 0 \\
\mu \mathrm{T})\end{array}$ & 6.4 & 7.1 & 7.8 & V \\
\hline Operating Current (at $0 \mathrm{mT}$ ) & & 16 & & $\mathrm{~mA}$ \\
\hline Supply Voltage Range & -0.3 & & 20 & $\mathrm{~V}$ \\
\hline Operating Supply Voltage & & 15 & & V \\
\hline $\begin{array}{l}\text { Operating Frequency (above }-3 \\
\qquad \mathrm{~dB})\end{array}$ & 0.1 & & 10000 & $\mathrm{~Hz}$ \\
\hline $\begin{array}{c}\text { Oscillator Square Wave Voltage } \\
\text { Range }\end{array}$ & 0 & & 5 & V \\
\hline $\begin{array}{l}\text { Oscillator Excitation Frequency } \\
\text { (Duty Cycle 50\%) }\end{array}$ & & 1 & & $\mathrm{MHz}$ \\
\hline Operating Temperature & -20 & & 50 & ${ }^{\circ} \mathrm{C}$ \\
\hline \multirow{2}{*}{ Noise density (in air, at $1 \mathrm{~Hz}$ ) } & & 100 & & $\mathrm{nOe} / \sqrt{\mathrm{Hz}}$ \\
\hline & & 10 & & $\mathrm{pT} / \sqrt{\mathrm{Hz}}$ \\
\hline \multirow{2}{*}{$\begin{array}{l}\text { Peak-to-Peak Noise (resolution } \\
\text { at full bandwidth) } \\
\text { (in air) }\end{array}$} & & \pm 3 & \pm 10 & $\mu \mathrm{Oe}$ \\
\hline & & \pm 0.3 & \pm 1.0 & $\mathrm{nT}$ \\
\hline
\end{tabular}

A preliminary characterization of the sensitivity curve for the new GMI sensor prototypes was performed using a Helmholtz coil to generate the reference magnetic flux density. The coil used in the laboratory follows eq. (97), where $B_{H}$ is the magnetic flux density at the center of the Helmholtz coil, in T, and $I_{H}$ is the 
current flowing through the coil, in A. The magnetic field can be easily controlled by using a voltage source $V_{\text {Hin }}$ and a series resistor $R_{S}$, as well as the knowledge of the coil impedance $Z_{H}$.

$$
B_{H}=2.87 \cdot 10^{-4} \cdot I_{H}=2.87 \cdot 10^{-4} \cdot \frac{V_{H i n}}{Z_{H}+R_{S}}
$$

The values for the impedance $Z_{H}$ in $\Omega$ were obtained for varying frequencies by experimental studies [26], and can be approximately given by

$$
Z_{H}=10^{-10} \cdot f^{3}-2 \cdot 10^{-6} \cdot f^{2}+0.0257 \cdot f+3.9671 .
$$

Measurements were performed on a hysteresis cycle, using the average values obtained for the GMI sensor voltage output. The results when using an excitation frequency of $8 \mathrm{kHz}$ are shown in Fig. 26.

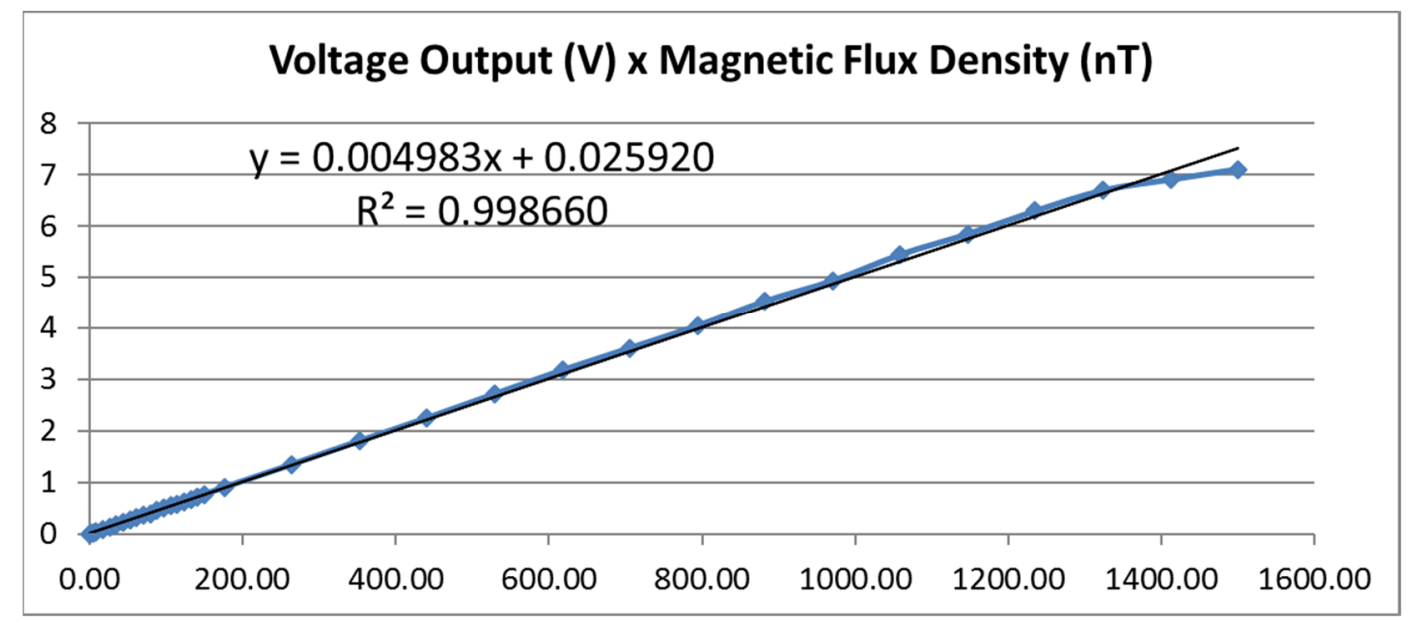

Figure 26 - Sensitivity curve for the GMI sensor Aichi MI-CB-1DJ-M-B obtained from characterization studies, given in voltage output versus magnetic flux density at the center of the Helmholtz coil for excitation frequency $f_{o}=8 \mathrm{kHz}$. Also displayed is the linear approximation for the curve.

These results are very promising, suggesting that the sensitivity is within the manufacturer specifications (typically $5.0 \mathrm{~V} / \mu \mathrm{T}$ ) and in fact maintains linearity up to $1.3 \mu \mathrm{T}$, while the specified linear region is up to $1.0 \mu \mathrm{T}$. Fig. 27 shows only the region considered approximately linear. 


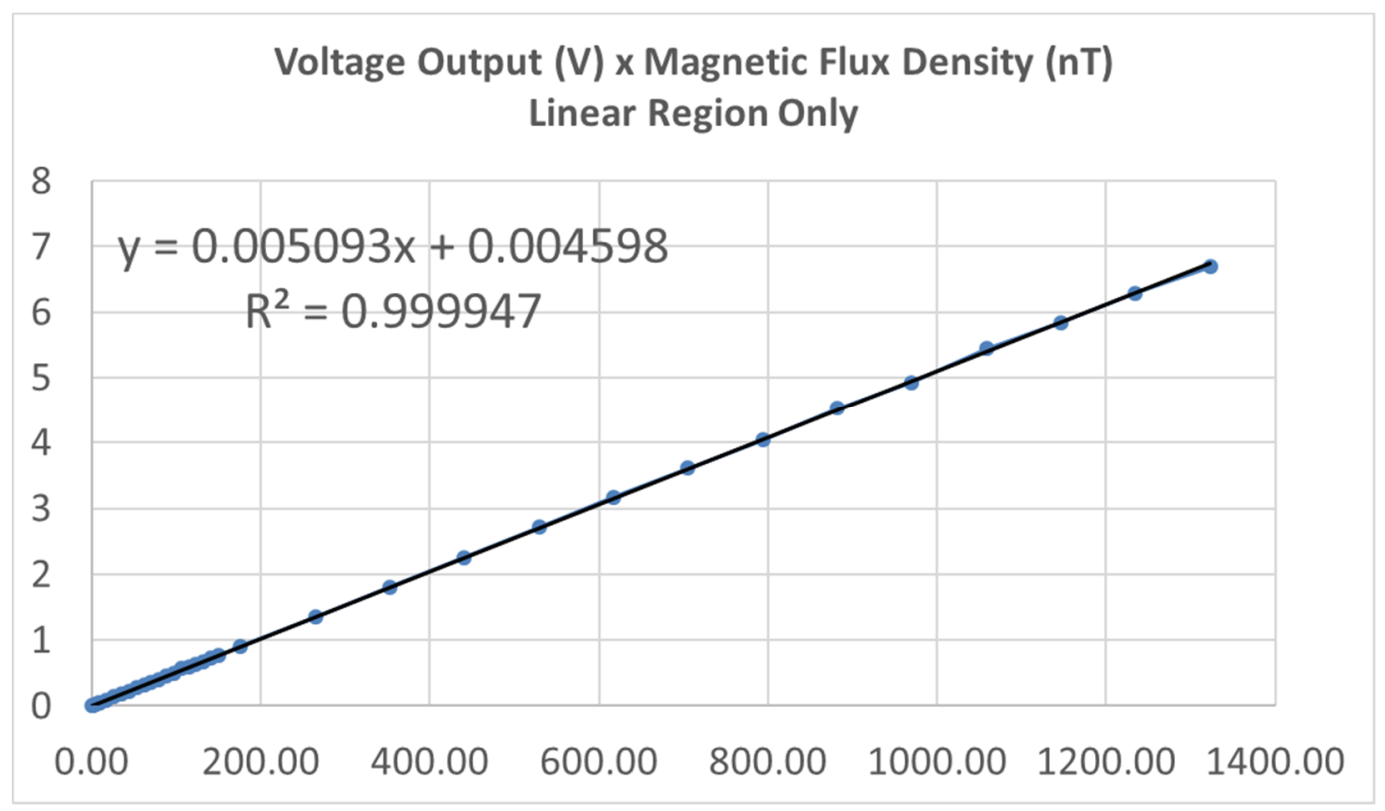

Figure 27 - Region considered linear for the sensitivity curve of the GMI sensor Aichi MI-CB-1DJ-M-B, again for $f_{o}=8 \mathrm{kHz}$.

The sensitivity obtained through this curve is $5.093 \mathrm{~V} / \mu \mathrm{T}$, a result in accordance with sensor specifications. This confirms the GMI sensor as an excellent option for foreign body detection.

\subsection{2.}

\section{Primary Magnetic Field Generator Design}

The main considerations for the solenoid design are spatial dimensions adequate for both GMI sensors, low excitation current to avoid limitations from the voltage signal source and a sufficiently high primary magnetic flux density, however preventing saturation of the GMI sensors at $\pm 1 \mu \mathrm{T}$.

Considering the GMI sensor dimensions in Fig. 25, the sensor elements (inside a $13.5 \mathrm{~mm}$ square) can be disposed symmetrically at the axis of a single layer solenoid with $70 \mathrm{~mm}$ length and $10 \mathrm{~mm}$ radius. The use of eq. (82) led to the decision of using 36 turns in order to obtain magnetic flux densities with absolute values around $1 \mu \mathrm{T}$ with low excitation currents around $2 \mathrm{~mA}$. The wire diameter can be up to $1.828 \mathrm{~mm}$ (13 AWG). An approximate value for the magnetic flux density at the center of the solenoid is given by

$$
B_{G M I} \approx 4 \pi \cdot 10^{-7} \frac{36 \cdot 2 \cdot 10^{-3}}{70 \cdot 10^{-3}} \approx 1.29 \mu \mathrm{T} .
$$


The non-negligible dimensions of the GMI sensor in relation to the solenoid require more detailed predictions for the magnetic fields inside the solenoid. Simulations were made on Matlab integrating the resulting magnetic flux density from each infinitesimal wire length to ensure suitable primary magnetic flux densities, especially over the sensor elements. Fig. 28 shows the solenoid modeled as a spiral in Matlab.

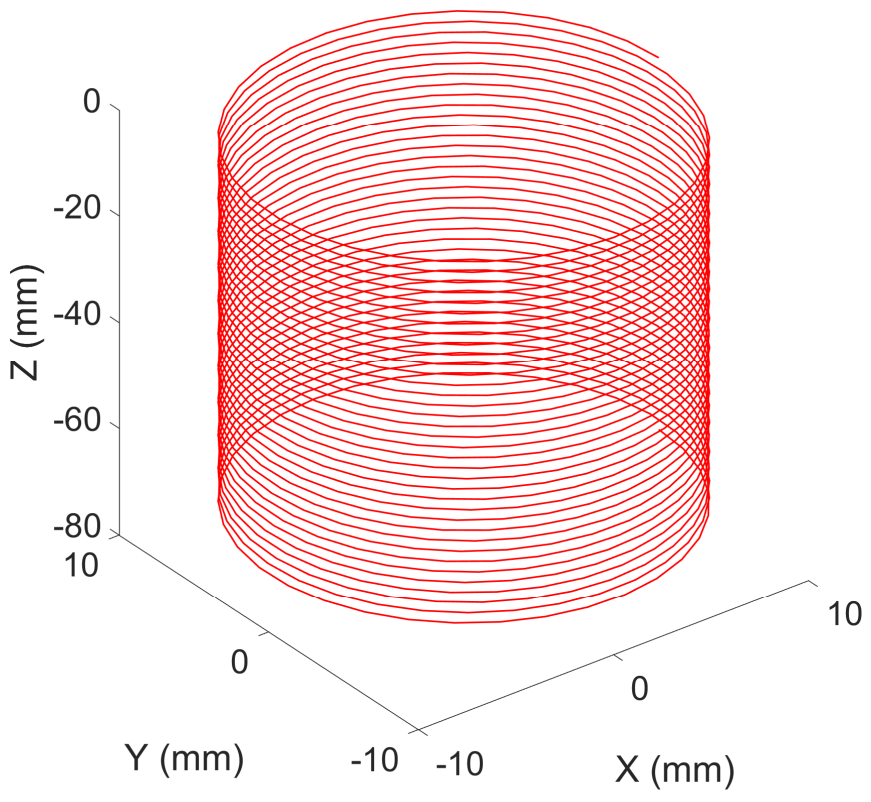

Figure 28 - Matlab model of the solenoid responsible for generating the primary magnetic field when using the GMI sensors. Spiral characteristics are $10 \mathrm{~mm}$ radius, $70 \mathrm{~mm}$ height and 36 turns evenly spaced.

The solenoid axis was placed along the $\mathrm{z}$-axis at $\mathrm{x}=0$ and $\mathrm{y}=0$. The edge of the solenoid that would be placed towards the foreign objects is at the $\mathrm{z}=0$ plane with the interior of the solenoid being at negative height values. Fig. 29 is a colormap plot showing the magnetic flux density behavior within the solenoid at the $\mathrm{x}=0$ plane. 


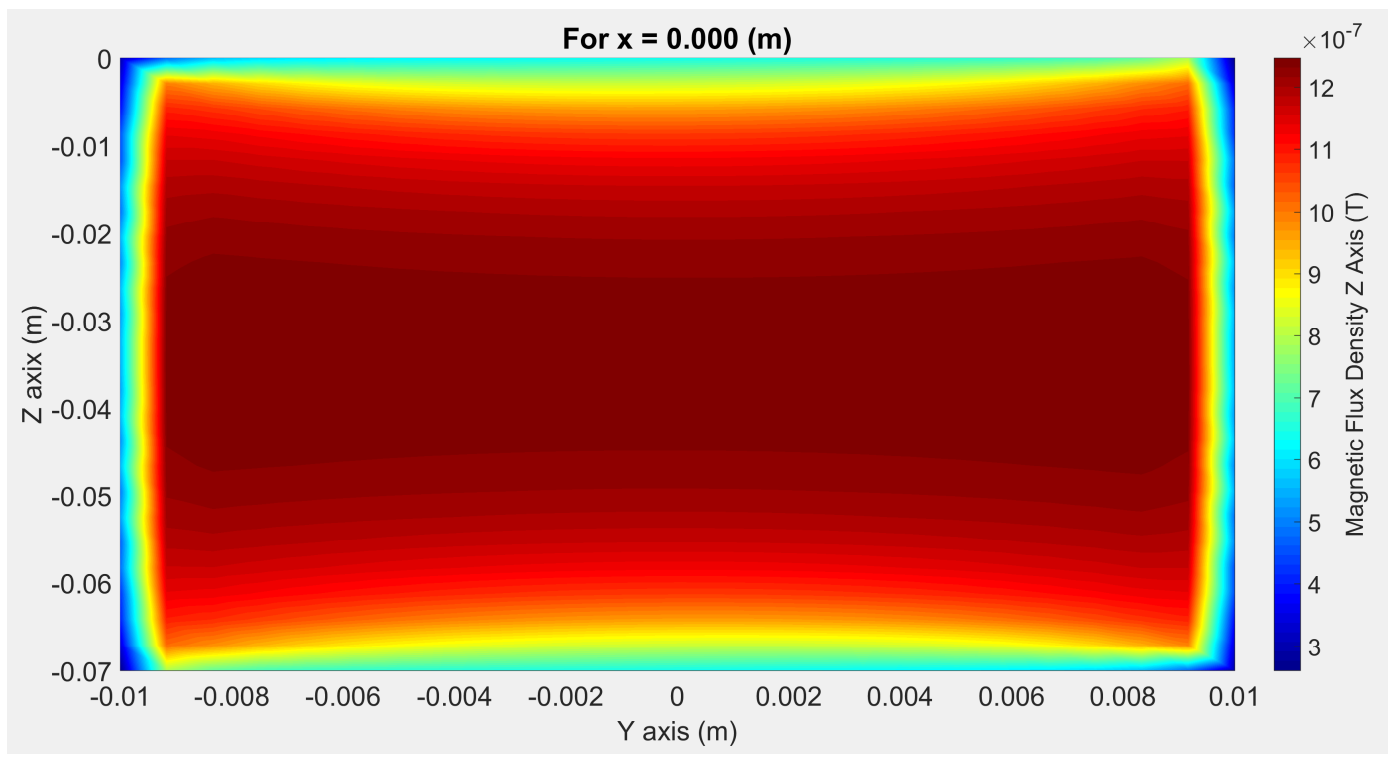

Figure 29 - Colormap of the magnetic flux density in the $z$ axis direction within the solenoid on the $x=0.0 \mathrm{~m}$ plane.

Fig. 30 presents the magnetic flux densities values along the z-axis when using a $2 \mathrm{~mA}$ excitation current.

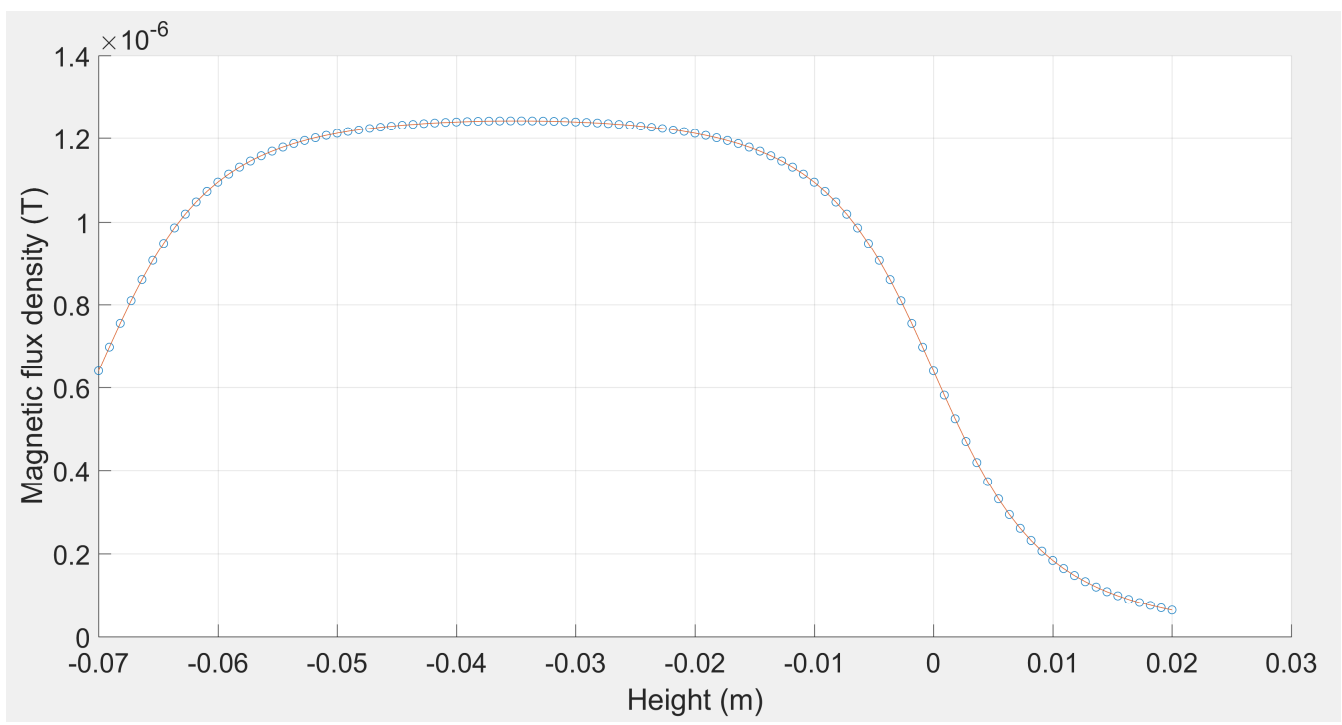

Figure 30 - Magnetic flux density on the solenoid axis for varying heights using a 2 mA excitation current.

As expected, the magnetic flux density values are higher and approximately constant around the middle of the solenoid, presenting symmetry around the central point $(\mathrm{z}=-35 \mathrm{~mm})$. The peak value is about $1.24 \mu \mathrm{T}$. Approaching the edges of the solenoid the magnetic field starts attenuating considerably, which allows the 
average value over each sensor element to be under $1 \mu \mathrm{T}$. At the edge of the solenoid the magnetic flux reaches approximately $640 \mathrm{nT}$.

Fig. 31 presents the average magnetic flux density over the superior sensor element for different excitation current values. This sensor is centered at $\mathrm{z}=-6.75 \mathrm{~mm}$. The same occurs in Fig. 32 for the inferior sensor, centered at $\mathrm{z}=-63.25 \mathrm{~mm}$. This establishes a base-line of approximately $56.5 \mathrm{~mm}$.

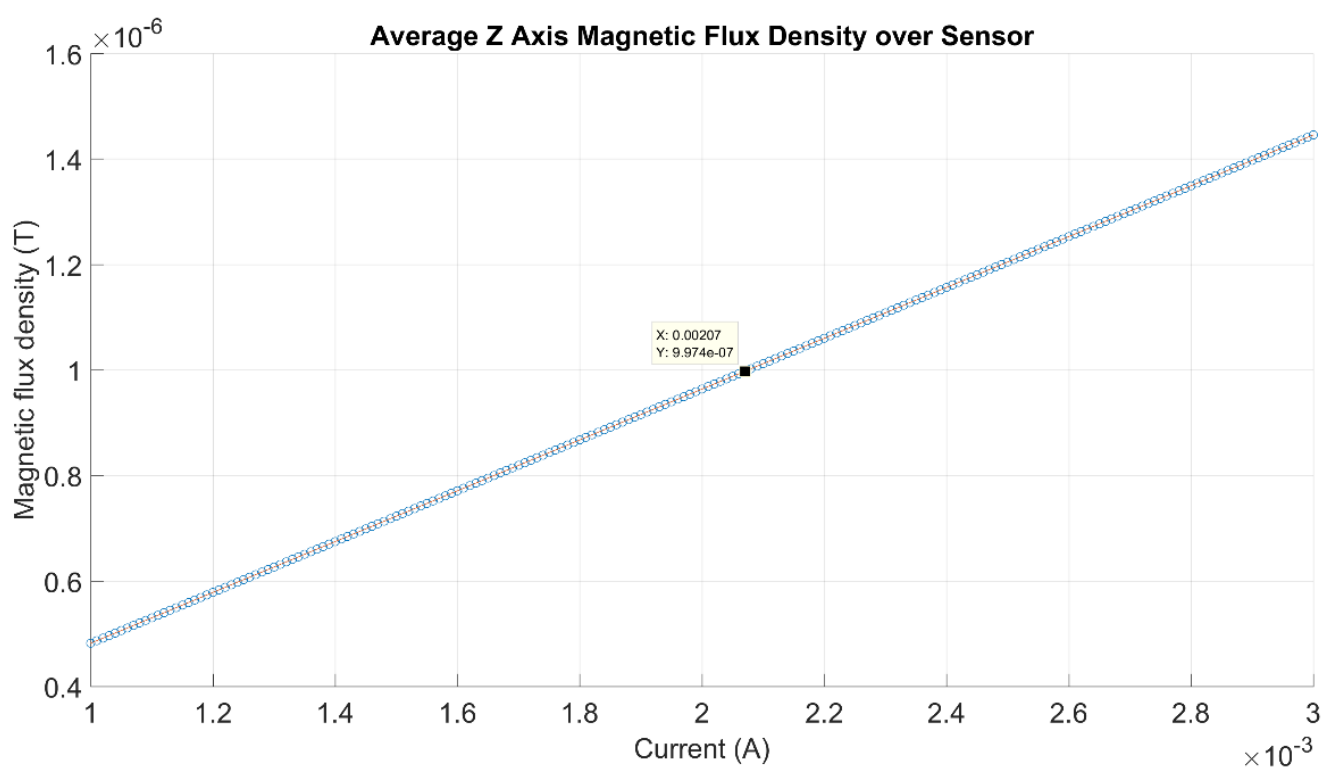

Figure 31 - Average magnetic flux density at the z-axis direction over the superior GMI sensor element, centered at approximately $z=-6.75 \mathbf{m m}$. 


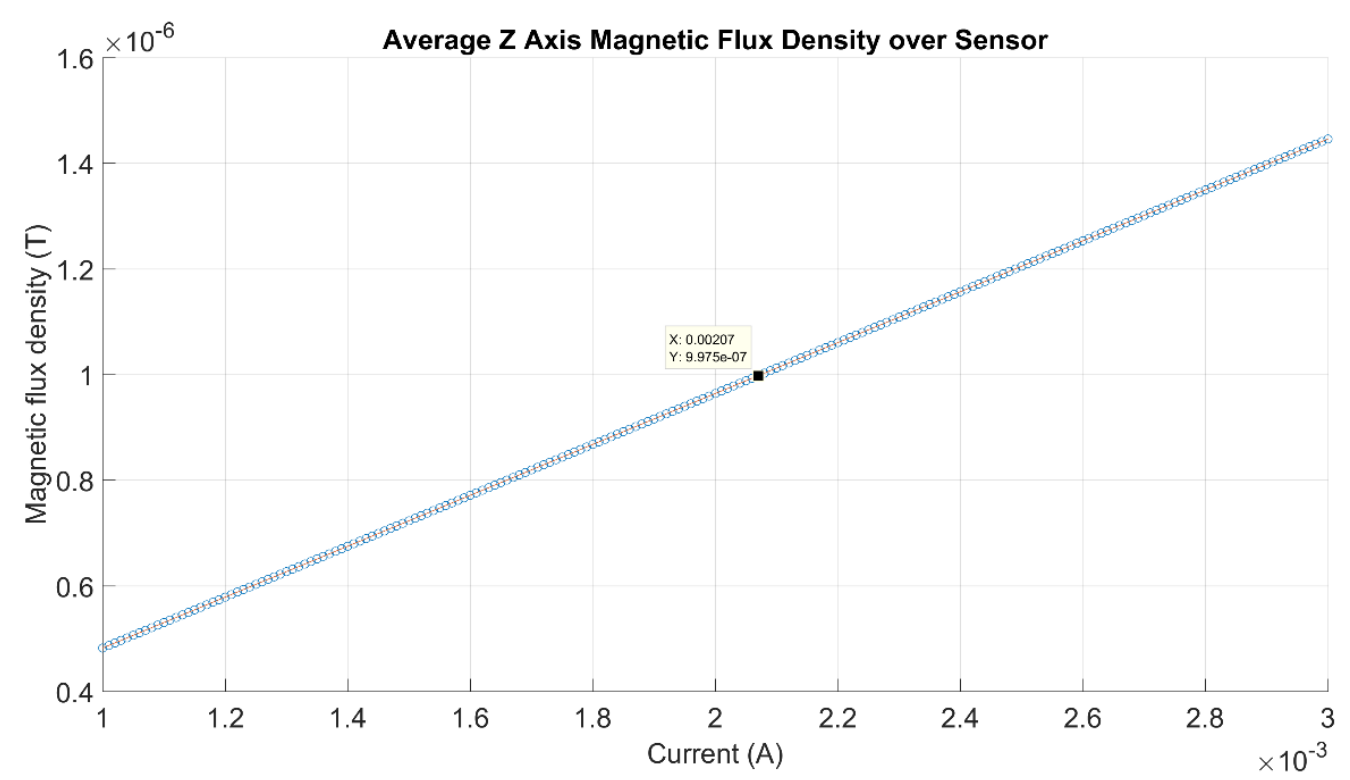

Figure 32 - Average magnetic flux density at the z-axis direction over the inferior GMI sensor element, centered at approximately $z=-63.25 \mathbf{m m}$.

According to Figs. 31 and 32, an excitation current of $2.07 \mathrm{~mA}$ leads to average magnetic flux densities under $998 \mathrm{nT}$ over the GMI sensors. This can be considered the superior limit for the excitation current due to the specified GMI sensor saturation. Using this current the magnetic flux density at the edge of the solenoid is around $660 \mathrm{nT}$.

\subsection{3.}

\section{Signal Processing Circuit for Gradiometric Reading}

The primary elements necessary for a GMI based detection system performance are bandpass filters for the sensor outputs and an instrumentation amplifier responsible by the gradiometric reading as well as signal amplification. The use of more selective filters is more important than in the GMR circuit due to the lower excitation frequency and also the lack of a DC bias.

Analysis of simulation results for different factors and different configurations lead to the selection of the topology to be implemented. Fig. 33 is a block diagram describing the main functional blocks of the proposed system.

Typically, rectifiers would be used before the instrumentation amplifiers inputs to avoid phase mismatch between the signals, however simulation studies showed that rectifying the signals would be prejudicial for the measurements of the secondary magnetic flux density. These results are presented in Appendix B. 


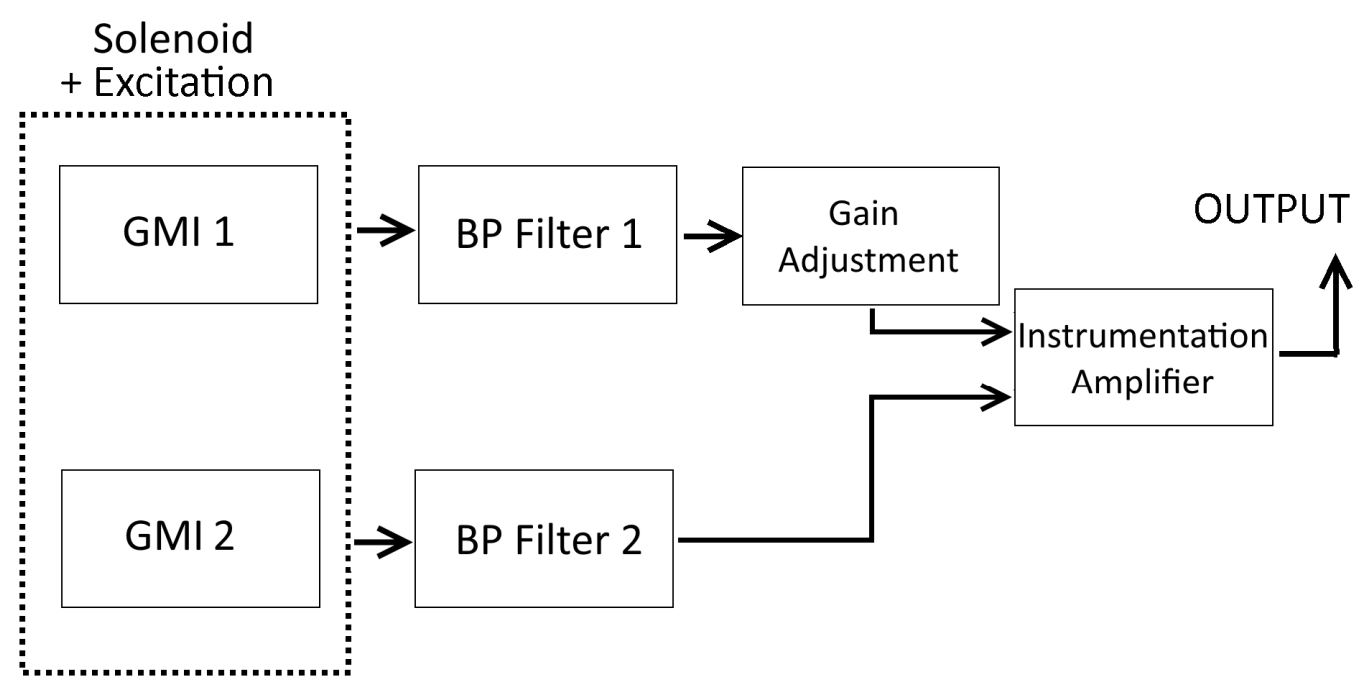

Figure 33 - Block diagram of the implemented circuit. "BP Filter" stands

\section{for bandpass filter.}

The solenoid excitation controls the frequency $f_{o}$ and amplitude $I_{o}$ of the solenoid current, responsible for generating the primary magnetic field. The GMI excitation is a power source of $15 \mathrm{~V}$.

BP stands for bandpass filters centered at $f_{o}$. The use of filters removes most environmental interference and minimizes electronic noise, in particular the $1 / f$ noise present in the sensor elements. This greatly improves the signal-to-noise ratio and allows better resolution.

The gain adjustment block consists of a controllable gain to one filter output in order to equalize both filters' amplitude responses, considering probable non-ideal characteristics to differ between filters. This stage is the intermediate processing stage of Fig. 11.

The instrumentation amplifier ideally outputs the subtraction between the input signals while introducing a gain. With GMI 1 being closer to the foreign body and GMI 2 being at a base-line $l_{s}$ distance from GMI 1, this characterizes the first order gradiometer configuration and the output is proportional to the secondary magnetic flux density. 


\subsubsection{1.}

\section{Bandpass Filters Topology}

In order to increase filter selectivity and keep passband gains with a flat response, Butterworth filters of $4^{\text {th }}$ order were selected to be implemented by a voltage controlled voltage source (VCVS) filter topology. This is often called a Sallen-Key bandpass filter. Fig. 34 introduces the topology for a second order filter. Cascading two second order stages creates a fourth order filter.

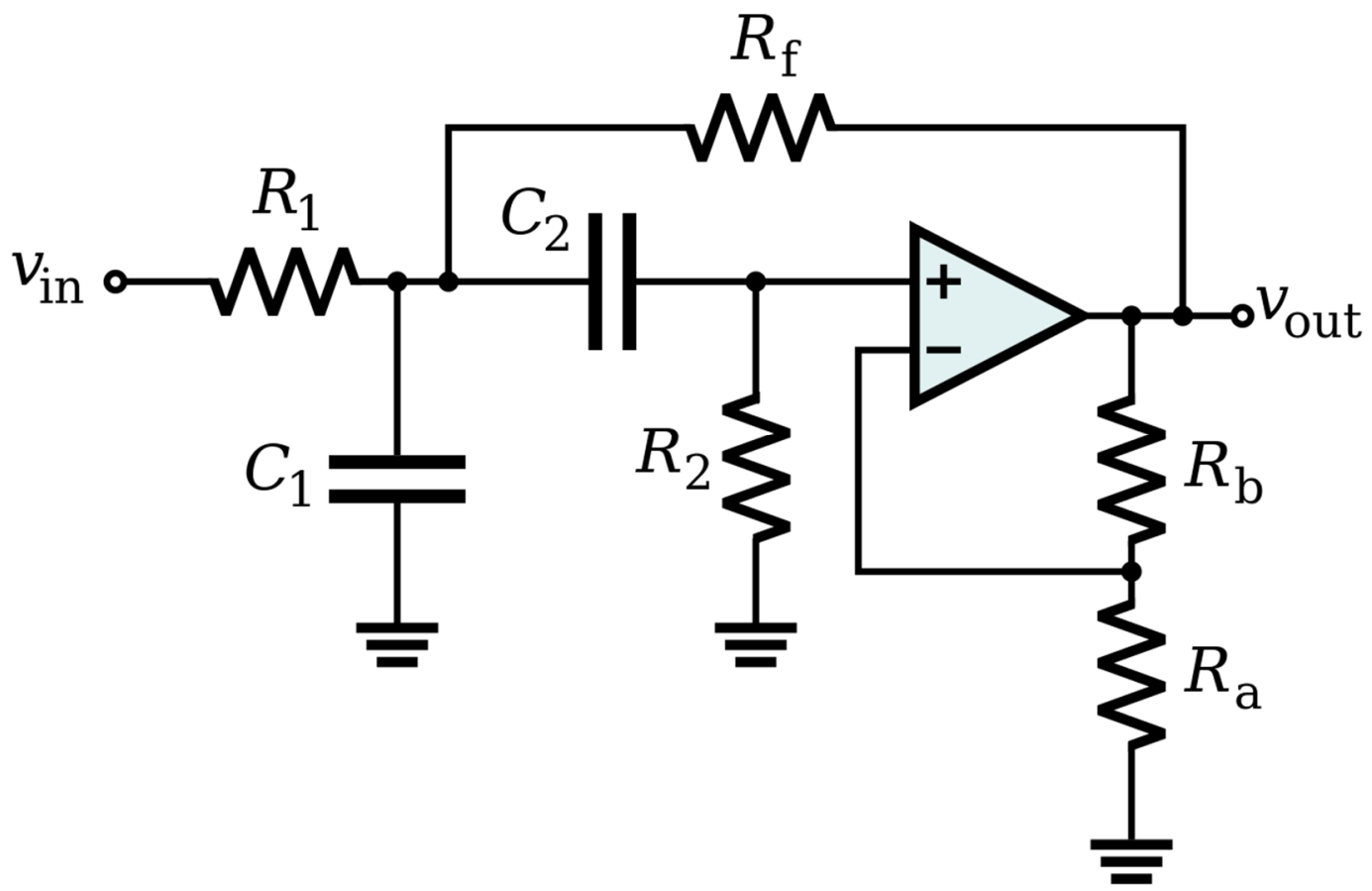

Figure 34 - VCVS bandpass filter topology (second order). [38]

The transfer function of the filter topology is given by

$$
H(s)=\frac{\left(1+\frac{R_{\mathrm{b}}}{R_{\mathrm{a}}}\right) \frac{s}{R_{1} C_{1}}}{s^{2}+\left(\frac{1}{R_{1} C_{1}}+\frac{1}{R_{2} C_{1}}+\frac{1}{R_{2} C_{2}}-\frac{R_{\mathrm{b}}}{R_{\mathrm{a}} R_{\mathrm{f}} C_{1}}\right) s+\frac{R_{1}+R_{\mathrm{f}}}{R_{1} R_{\mathrm{f}} R_{2} C_{1} C_{2}}} .
$$

The center frequency fo is given by

$$
f_{o}=\frac{1}{2 \pi} \sqrt{\frac{R_{1}+R_{\mathrm{f}}}{R_{1} R_{\mathrm{f}} R_{2} C_{1} C_{2}}}
$$

The $\mathrm{Q}$ factor is

$$
Q=\frac{\sqrt{\left(R_{1}+R_{\mathrm{f}}\right) R_{1} R_{\mathrm{f}} R_{2} C_{1} C_{2}}}{R_{1} R_{\mathrm{f}}\left(C_{1}+C_{2}\right)+R_{2} C_{2}\left(R_{\mathrm{f}}-R_{1} R_{\mathrm{b}} / R_{\mathrm{a}}\right)} .
$$


In order to ensure stability, the gain $\left(\mathbf{1}+\frac{\boldsymbol{R}_{\mathrm{b}}}{\boldsymbol{R}_{\mathrm{a}}}\right)$ should be smaller than 3 , but a gain closer to 1 is favored considering the possibility of saturating the instrumentation amplifier output.

Filter design software was used to aid the project. Associating the software projects with 1\% tolerance resistors, the filter shown in Fig. 35 was selected, using LM318 operational amplifiers.

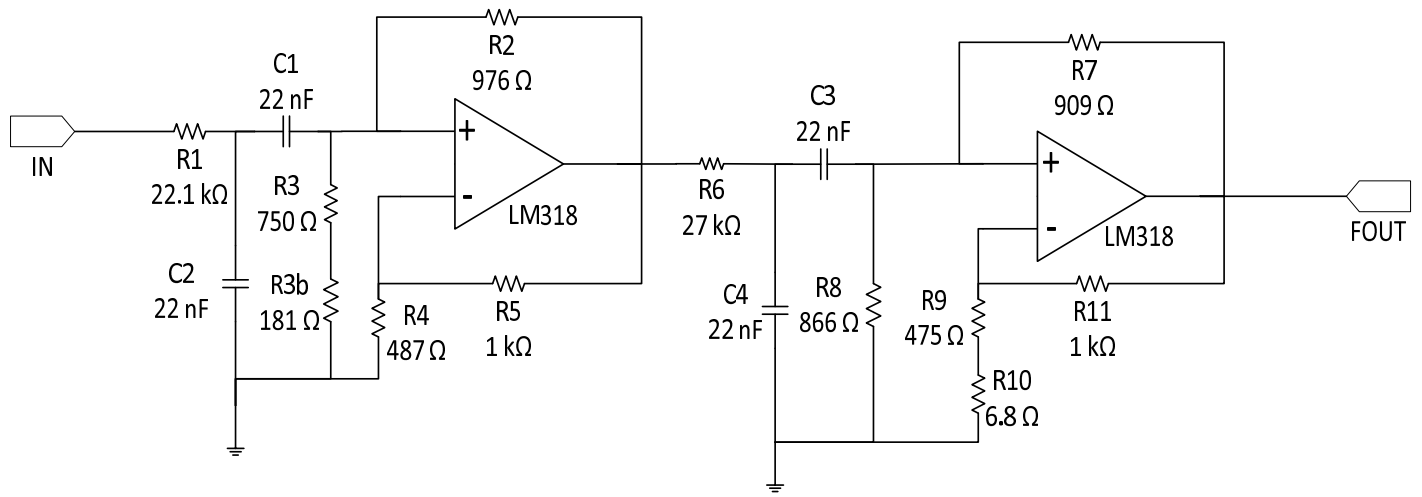

Figure 35 -Selected Fourth Order VCVS Bandpass Filter.

Fig. 36 shows this filter frequency response in magnitude with linear scale, as simulated by TopSpice, while Fig. 37 shows the filter frequency response in magnitude with $\mathrm{dB}$ scale as well as in phase. 
TopSPICE 7.17e

03-JUL-2016

18:18:59

- M[GAIN]

Cursor 1:

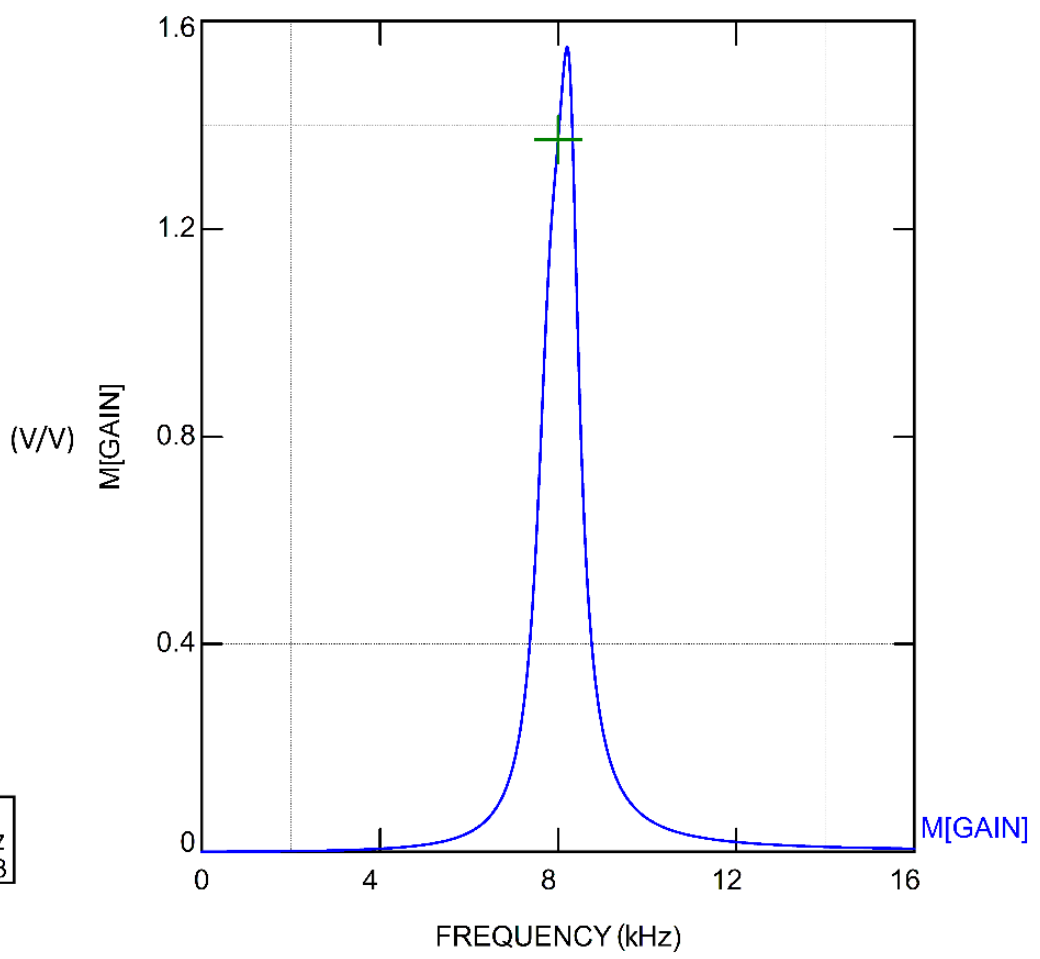

Figure 36 - Bandpass filter simulated frequency response in magnitude (linear scale).
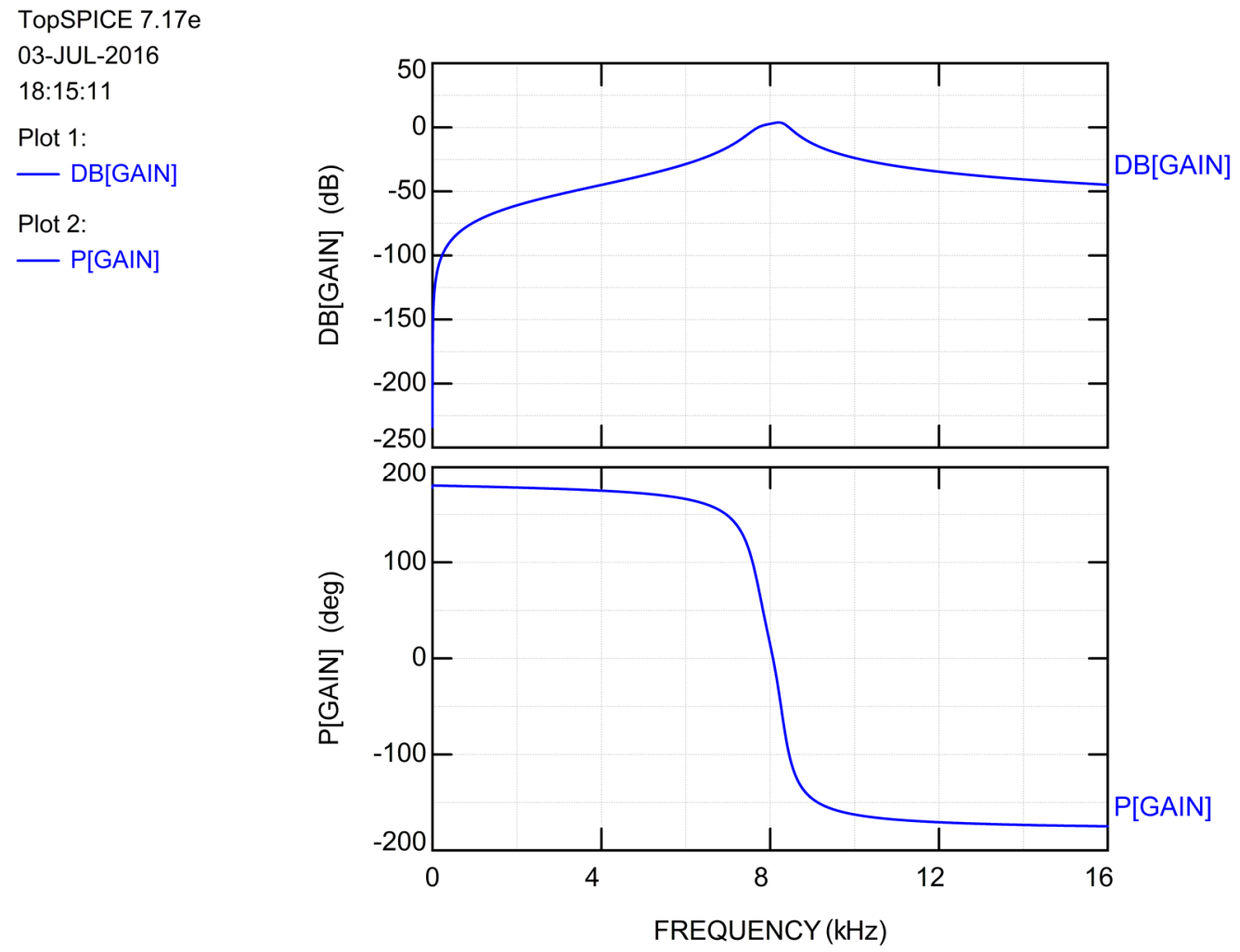

Figure 37 - Bandpass filter simulated frequency response in magnitude (dB scale) and phase. 
The response is shown to be slightly off the expected center frequency of $8 \mathrm{kHz}$. The gain at $8 \mathrm{kHz}$ is about 1.37 while the maximum gain is closer to 1.5 . Fig. 38 shows a closer look at the filter passband.
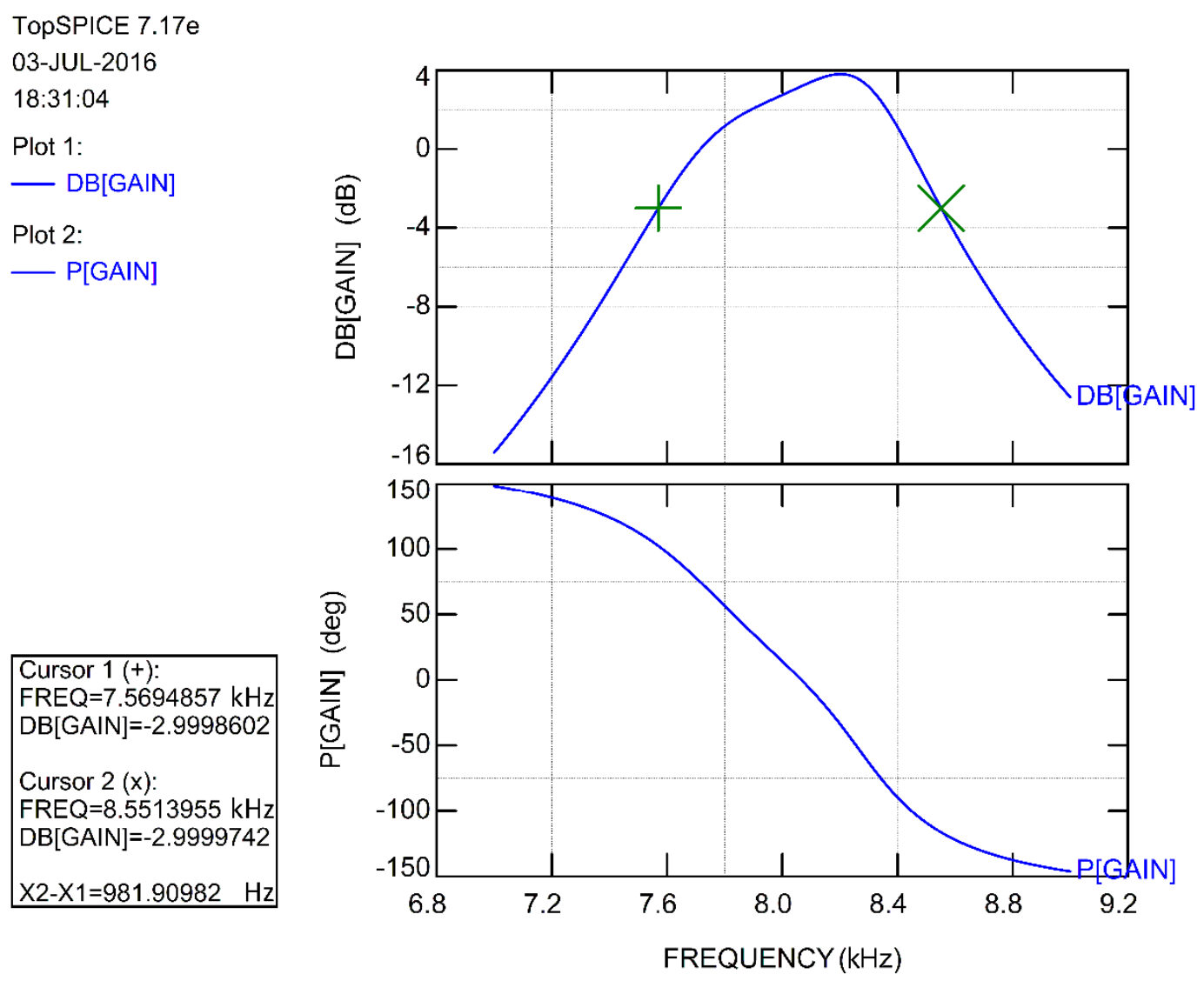

Figure 38 - Bandpass filter simulated frequency response in magnitude (dB scale) and phase, focusing on the passband. The cutoff frequencies are marked with the cursors.

Here the cutoff frequencies are estimated as $7569 \mathrm{~Hz}$ and $8551 \mathrm{~Hz}$, with the bandwidth given as about $982 \mathrm{~Hz}$.

An experimental prototype of the filter was made and characterized using a signal generator with frequency sweep mode and a digital oscilloscope in X-Y configuration. Results were recorded in text files and post-processed in Matlab, generating Fig. 39 in linear scale and Fig. 40 in $\mathrm{dB}$ scale. In both figures the orange line represents the TopSpice simulations for comparison. 


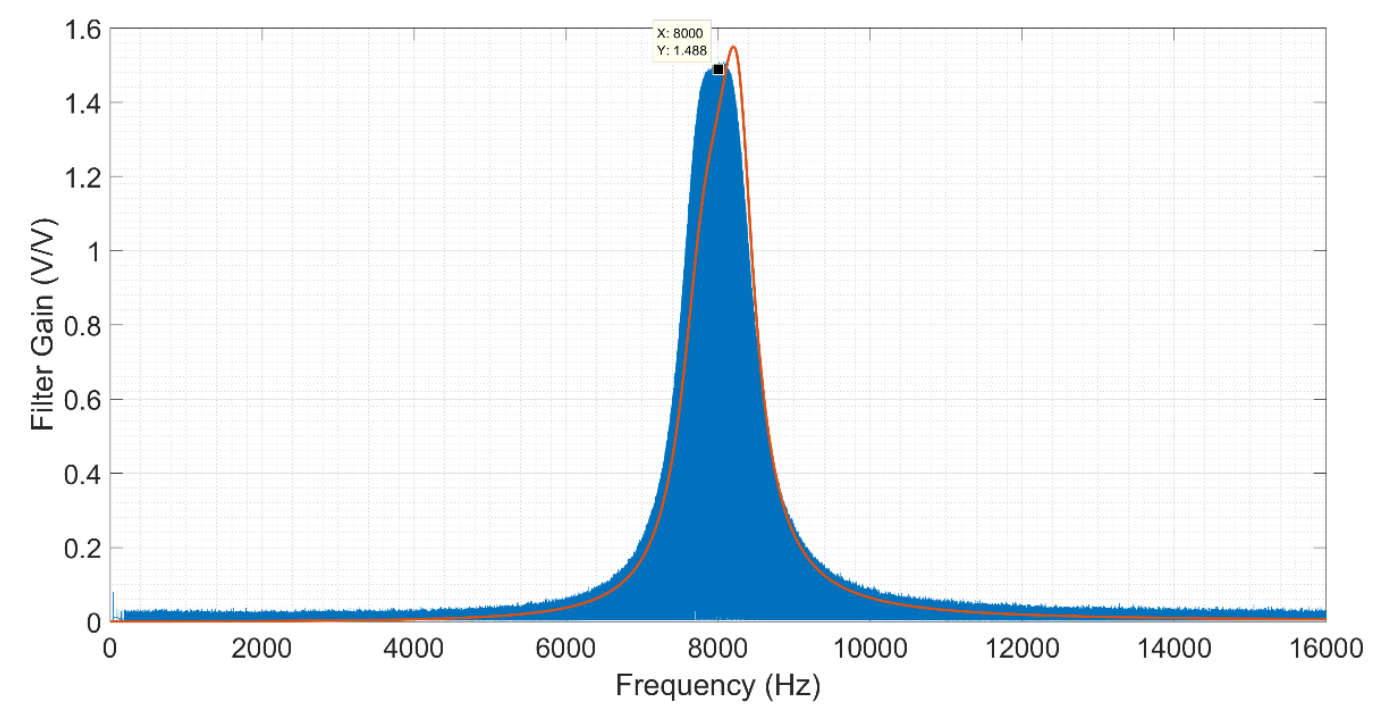

Figure 39 - Results of prototype filter characterization in magnitude frequency response (linear scale) in blue. The orange line is the simulated response.

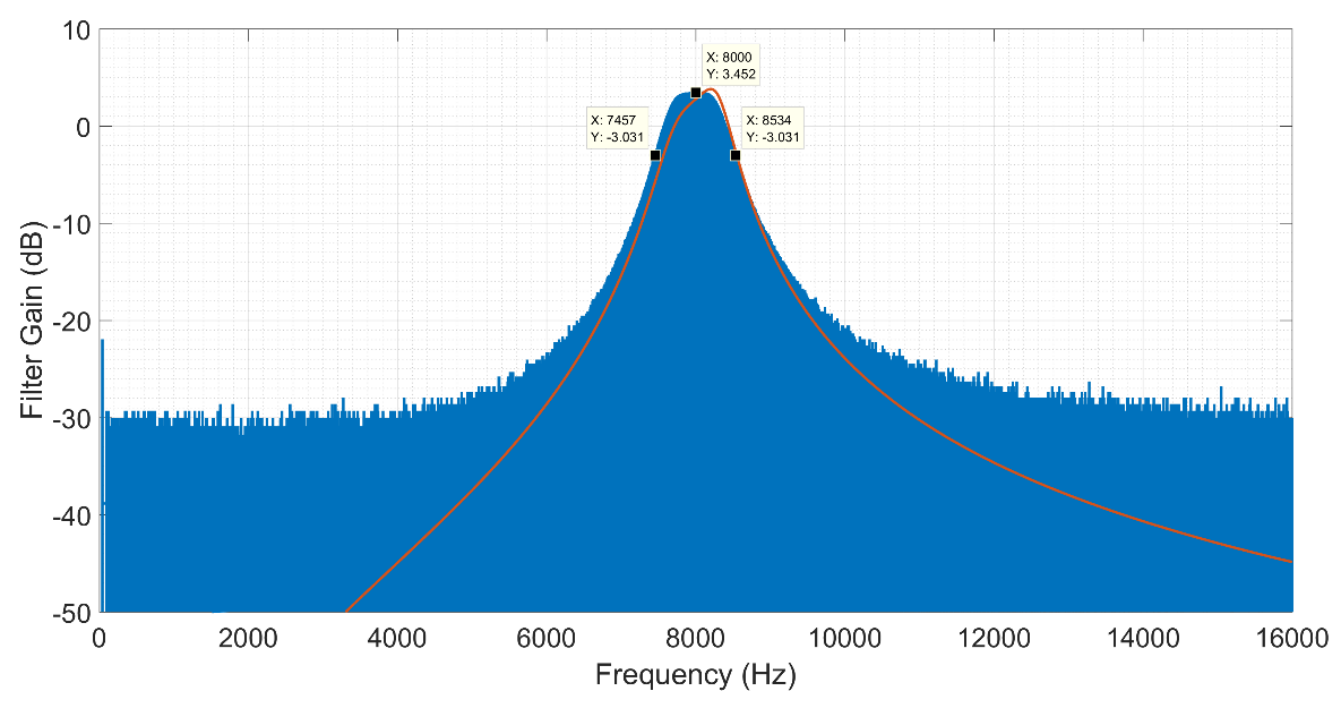

Figure 40 - Results of prototype filter characterization in magnitude frequency response ( $\mathrm{dB}$ scale) in blue. The orange line is the simulated response.

The oscilloscope data suggests the prototype filter has a flatter passband and is closer to the desired center frequency of $8 \mathrm{kHz}$. The gain at $8 \mathrm{kHz}$ is approximately $1.488 \mathrm{~V} / \mathrm{V}$ or $3.452 \mathrm{~dB}$. The cutoff frequencies are close to $7457 \mathrm{~Hz}$ and $8534 \mathrm{~Hz}$, which result in a bandwidth of about $1077 \mathrm{~Hz}$. The rejection bands contain higher 
gains than the simulated model, which is expected considering the diverse noise sources in the laboratory and non-ideal aspects of the equipment utilized.

The characterization studies suggest the filter succeeds in its goals, in fact performing closer to some desired characteristics (passband flatness and center frequency) than the simulation results.

The two separate but theoretically identical filters were then implemented in a universal circuit board. Preliminary tests using a signal generator, an oscilloscope and a frequency meter showed each filter has a slightly different response. For a same input signal with frequency around $8.8 \mathrm{kHz}$, both filters exhibit approximately the same output gain but a phase mismatch close to 1 degree. Meanwhile, setting the input frequency around $7.7 \mathrm{kHz}$ minimizes the phase mismatch to 0.030 degree while presenting a gain difference around $30 \%$.

Considering the good performance of the detection system relies on the signals originating from the primary magnetic flux density being in phase and implementing phase correction circuits would require more complex designs, using a frequency close to $7.7 \mathrm{kHz}$ is preferred. The output gain difference can be easily corrected.

\subsubsection{2. Prototype Configuration}

In order to compensate for the different filter gains at the new excitation frequency, a passive gain adjustment block is implemented as a resistive voltage divider with the variable output controlled by a potentiometer. The schematic for the location system prototype is presented in Fig. 41: 


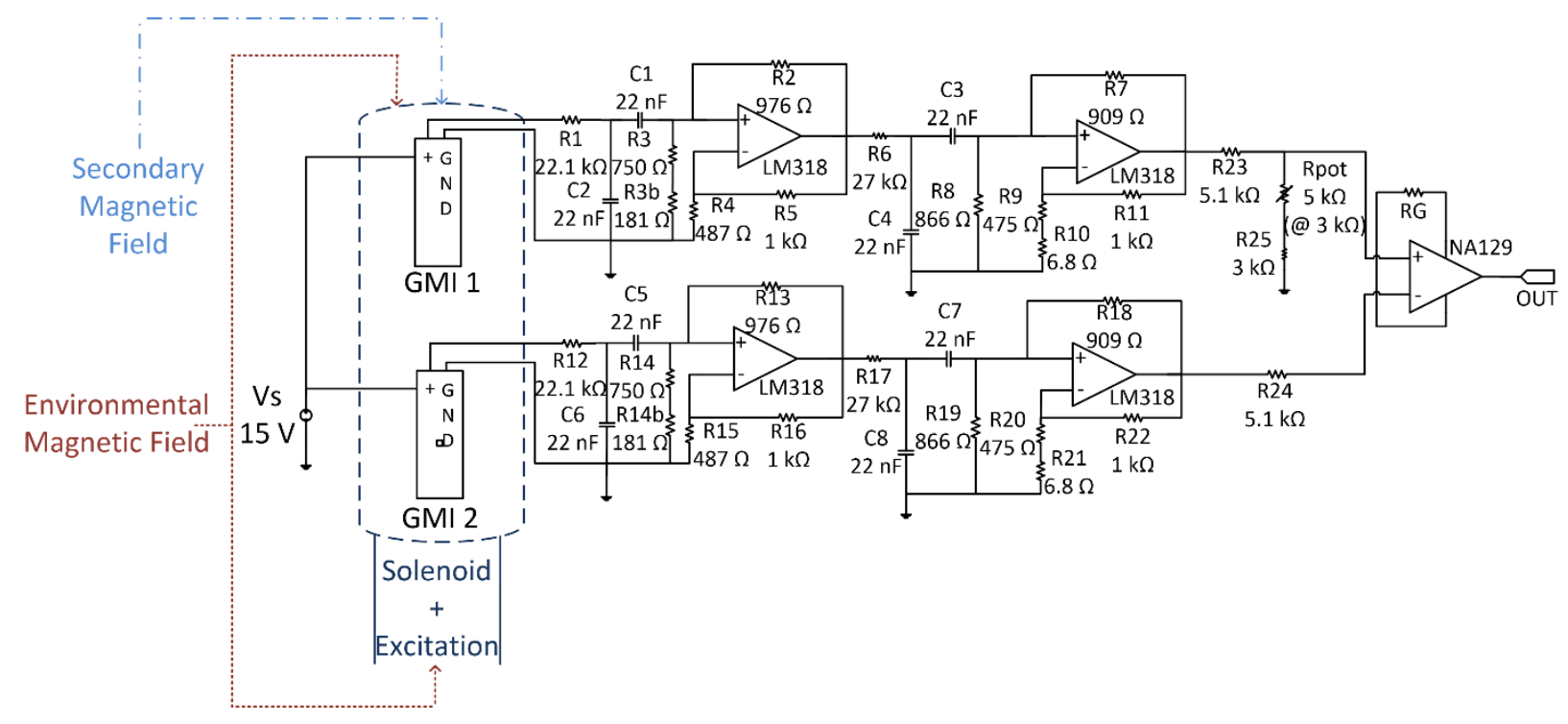

Figure 41 - Schematic for the detection system prototype.

Each GMI sensor output is connected to a $4^{\text {th }}$ order bandpass filter. The filtered signals are inputs to an instrumentation amplifier to perform the gradiometric reading. The instrumentation amplifier gain resistor can be exchanged to allow different measuring ranges. Since these GMI sensors require a DC voltage source of $15 \mathrm{~V}$, the amplifiers are powered by $\pm 15 \mathrm{~V}$.

Considering the output amplitude from the GMI sensors is expected to be around $5 \mathrm{~V}$ and the selected differential gain is typically close to $99.8 \mathrm{~V} / \mathrm{V}$, the CMRR is expected to be around $90 \mathrm{~dB}$. Thus, the predicted maximum signal of interest $V_{\text {dmax }}$ before saturation is

$$
\begin{aligned}
V_{\text {max }}=13.75 & \approx 99.8\left(V_{d \max }\right)+0.00316 \frac{\left(2 \cdot 5+V_{d \max }\right)}{2} \\
V_{d \max } & \approx \frac{13.75-0.0158}{99.8+0.00158}=137.61 \mathrm{mV} .
\end{aligned}
$$

This signal is associated with an approximately $27.5 \mathrm{nT}$ magnetic flux density for the secondary magnetic field. Similar procedures can be used to obtain maximum values for other differential gains, allowing different reading ranges for the same circuit. Table 5 shows the maximum signal of interest in nT for several differential gains. 
Table 5 - Maximum signal of interest for several differential gains applied in the GMI detection system.

\begin{tabular}{|c|c|c|}
\hline Gain Resistor $\boldsymbol{R}_{\boldsymbol{G}}(\boldsymbol{\Omega})$ & $\begin{array}{c}\text { Differential Gain } \boldsymbol{G}_{\boldsymbol{d i f}} \\
\text { (V/V) }\end{array}$ & $\begin{array}{c}\text { Maximum Secondary } \\
\text { Magnetic Flux Density } \\
\text { (nT) }\end{array}$ \\
\hline 50000 & 1.988 & 1383.29 \\
\hline 5000 & 10.88 & 252 \\
\hline 500 & 99.8 & 27.5 \\
\hline 50 & 989 & 2.77 \\
\hline
\end{tabular}

The minimum values are more difficult to assess without simulations and experimental results, depending mostly on the circuit bandwidth for noise considerations and also on non-ideal parameters of several circuit components that can limit the linearity of the output for very low signals, impacting the resolution of the device. However, considering the high CMRR at the utilized frequencies, the common-mode output would be negligible.

\subsubsection{3.}

\section{Estimated Resolution}

The GMI sensor possesses a very low noise density at $10 \mathrm{pT} / \sqrt{\mathrm{Hz}}$, which associated with a sufficiently narrow bandwidth is expected to result in a very good magnetic flux density resolution. Using the sensitivity of $5 \mathrm{~V} / \mu \mathrm{T}$, the equivalent spectral power density would be $2.5 \mathrm{nW} / \mathrm{Hz}$. Integrating this noise spectral power density and extracting its root results in the noise for the circuit in root mean square value (rms), such as

$$
\text { NoiseGMI } I_{r m s}=\frac{1}{\operatorname{Sens}} \sqrt{\int_{1.57 \cdot f 1}^{1.57 \cdot f 2} \frac{s_{\text {onoise }}}{f} d f}=\frac{1}{5 \cdot 10^{6}} \sqrt{\int_{1.025 \cdot 7457}^{1.025 \cdot 8534} \frac{2.5 \cdot 10^{-9}}{f} d f}=3.67 \mathrm{pT} .
$$

The Equivalent Noise Bandwidth (ENB) factor for a fourth order Butterworth filter is 1.025 . This noise is equivalent to an rms voltage of $18.36 \mu \mathrm{V}$. This is a considerable noise voltage level for a narrow bandwidth, suggesting it is the main 
factor in the system resolution. Using the 6.6 conversion factor from rms value to peak-to-peak with $99.9 \%$ confidence leads to

$$
\text { NoiseGMI }_{p p}=6.6 \cdot \text { NoiseGMI }_{r m s}=24.2 \mathrm{pT} \approx 25 \mathrm{pT} .
$$

This would be the best resolution considering only the sensor at this bandwidth. It is a great improvement over what the GMR can offer, which would be around $110 \mathrm{nT}$ for this same bandwidth.

In order to verify if the noise due to other components would be significant, a simplified analysis can be performed. First, the noise at the input of the LM318 amplifiers would follow

$$
v_{n L M 318}=\sqrt{\left(60 \cdot 10^{-9} \mathrm{~V} / \sqrt{\mathrm{Hz}}\right)^{2} \cdot 1.025 \cdot(1077 \mathrm{~Hz})}=1.99 \mu \mathrm{V} \approx 2.0 \mu \mathrm{V} .
$$

The noise voltage due to the LM318 amplifier is about 10 times smaller than the sensor noise, therefore it is negligible.

The highest thermal noise would be due to a $27 \mathrm{k} \Omega$ resistor, given by

$$
v_{n}=\sqrt{4 k_{B} T R B}=\sqrt{4 \cdot 1.38 \cdot 10^{-23} \mathrm{~J} / \mathrm{K} \cdot 298.15 \mathrm{~K} \cdot 27000 \Omega \cdot 1.025 \cdot(1077 \mathrm{~Hz})}=700 \mathrm{nV} .
$$

Thus the thermal noise due to a single resistor is at least 26 times smaller than the sensor output noise. Analyzing the resulting noise due to all the resistors used would be computationally demanding and needless since these noise sources are negligible.

The noise at the inputs of the instrumentation amplifier would only appear after the filters, but their voltage level in a worst case scenario (smallest gain), would be given by

$$
v_{\text {INA129 }}=\sqrt{\left(110 \cdot 10^{-9} \mathrm{~V} / \sqrt{\mathrm{Hz}}\right)^{2} 1.025 \cdot(1077 \mathrm{~Hz})}=3.65 \mu \mathrm{V} .
$$

Once again, this worst case scenario noise is almost 5 times smaller than the sensor output noise, being negligible. In conclusion, the estimated resolution for the complete system is approximately $25 \mathrm{pT}$.

\subsubsection{4.}

\section{Estimated Minimum Detectable Diameter}

Using the primary magnetic field generator values with the model for the peak secondary magnetic flux density due to the eddy currents on foreign bodies of various radii leads to the results in Fig. 42. 


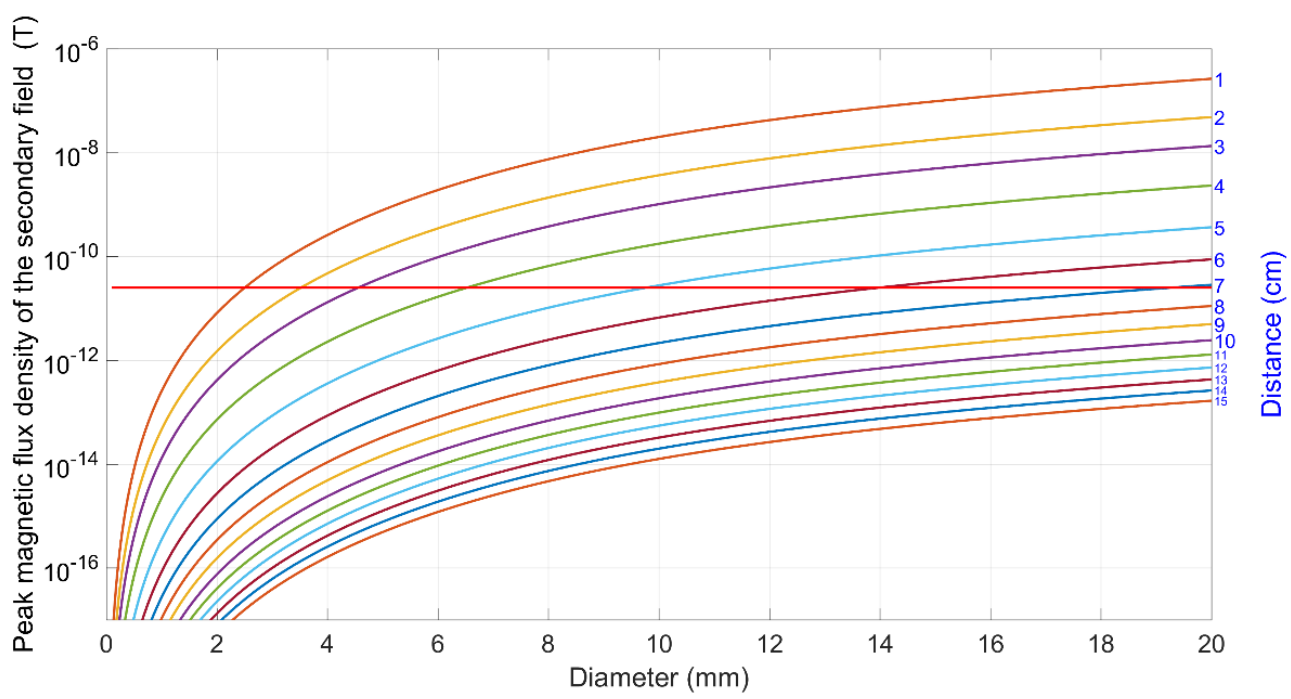

Figure 42 - Curves of peak secondary magnetic flux density magnitude (Bmax) versus the diameter of the foreign body for different heights. The blue number on the right indicates the height value in $\mathrm{cm}$ of the respective curve.

The reference line represents the estimated resolution of $25 \mathrm{pT}$.

The red line stands for the estimated detection system resolution values. Fig. 43 shows the minimum detectable diameter varying with the height when assuming this $25 \mathrm{pT}$ resolution and compares it to the GMR sensor considering its own estimated resolution of $2 \mu \mathrm{T}$.

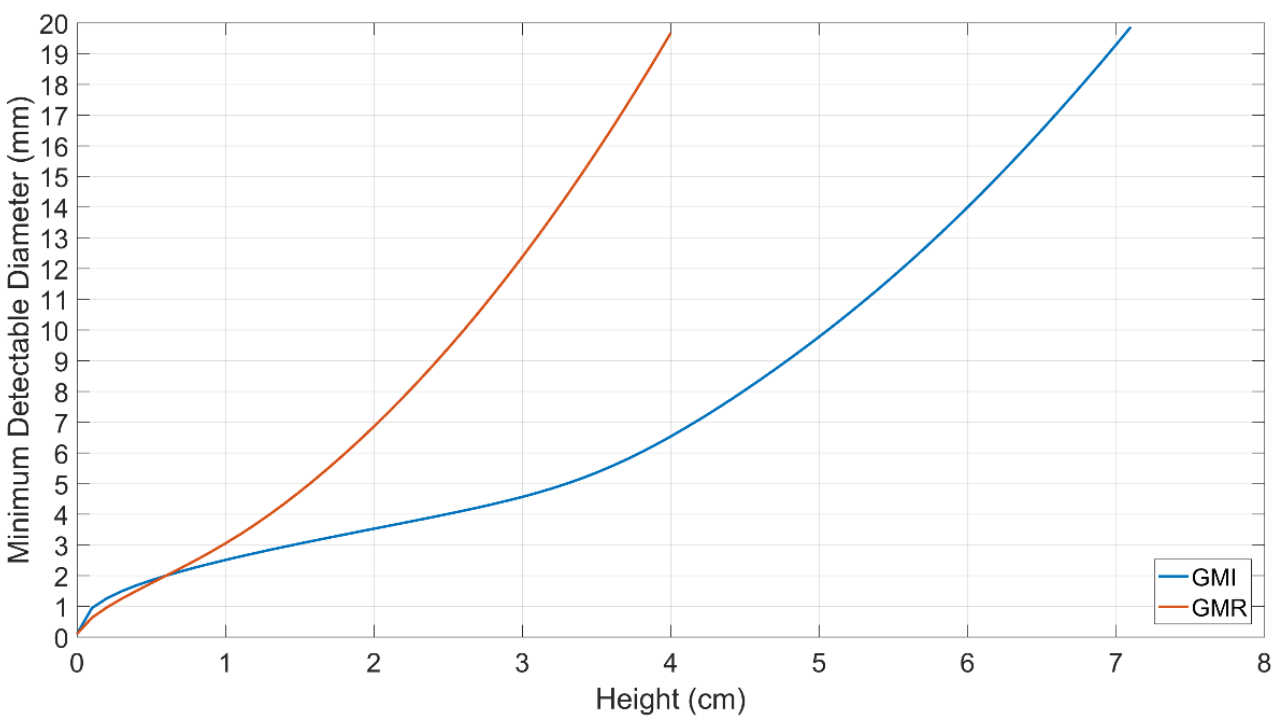

Figure 43 - Comparison of GMI and GMR performance regarding minimum detectable diameter over height using the estimated resolutions for each detection system ( $2 \mu \mathrm{T}$ for the GMR system and 25 pT for the GMI system). 
The GMR sensor performs slightly better at heights under $60 \mathrm{~mm}$ due to the considerably higher primary magnetic fields, while the GMI sensitivity and resolution lead to an improved detection range up to $7 \mathrm{~cm}$. For distances larger than $2 \mathrm{~cm}$, the minimum detectable diameter is at least halved when using the GMI sensor.

The gains in performance with the GMI sensor, as well as the reduced complexity and increased safety resultant of the simplified solenoid excitation justify the shift to this new sensor prototype.

\subsubsection{5.}

\section{Simulation Results}

The results from TopSpice simulations are presented in Figs. 44 - 47 for four different instrumentation amplifier gains.

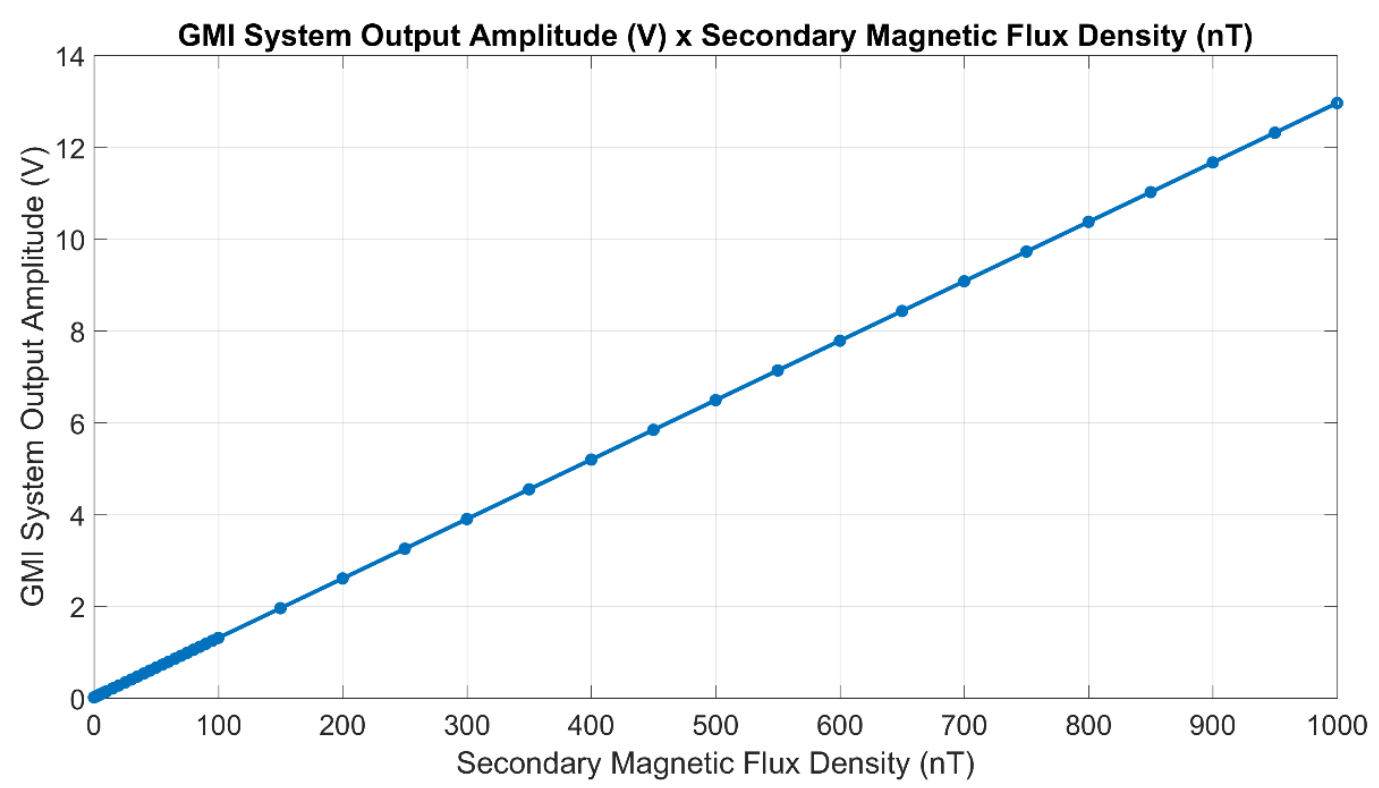

Figure 44 - GMI system output amplitude (V) for gain of $1.988 \mathrm{~V} / \mathrm{V}$ for secondary magnetic flux densities varying from 0 to $1000 \mathrm{nT}$. 


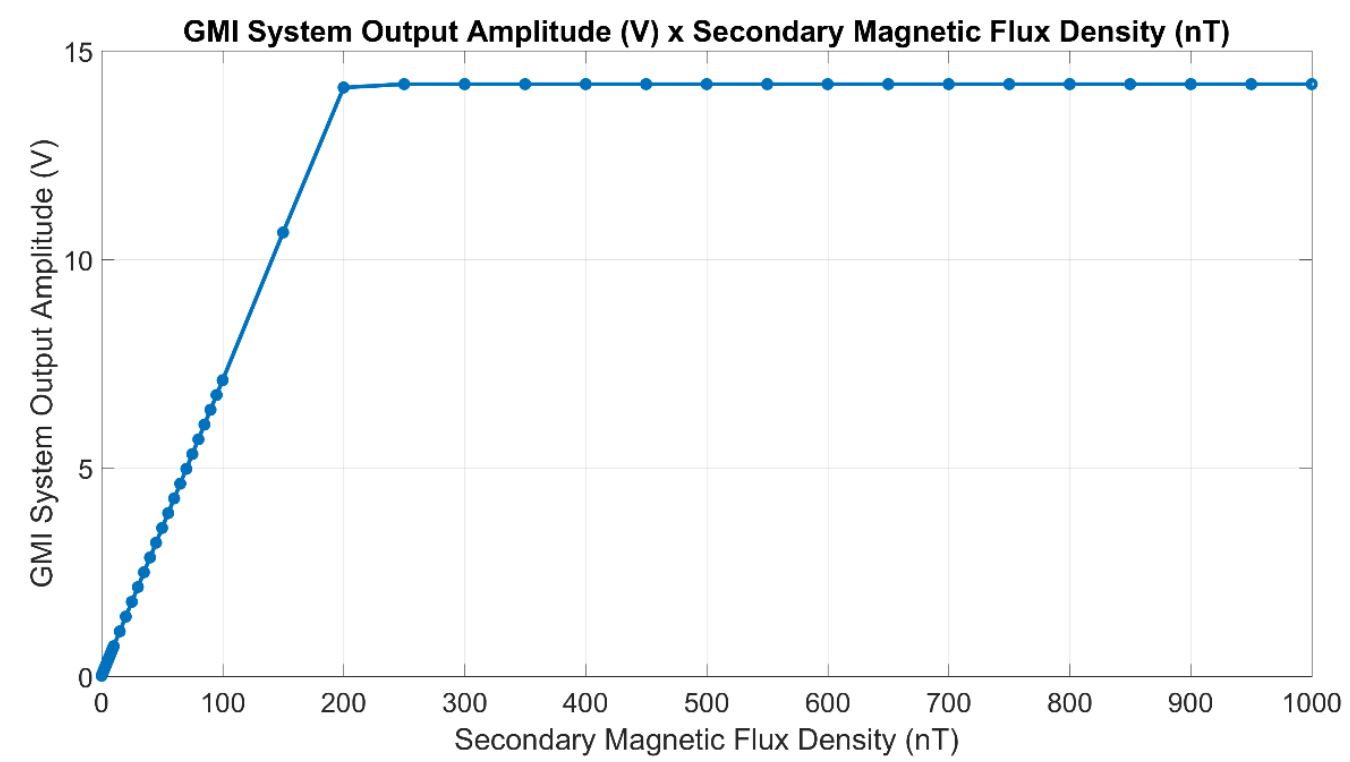

Figure 45 - GMI system output amplitude (V) for gain of $10.88 \mathrm{~V} / \mathrm{V}$ for secondary magnetic flux densities varying from 0 to $1000 \mathrm{nT}$.

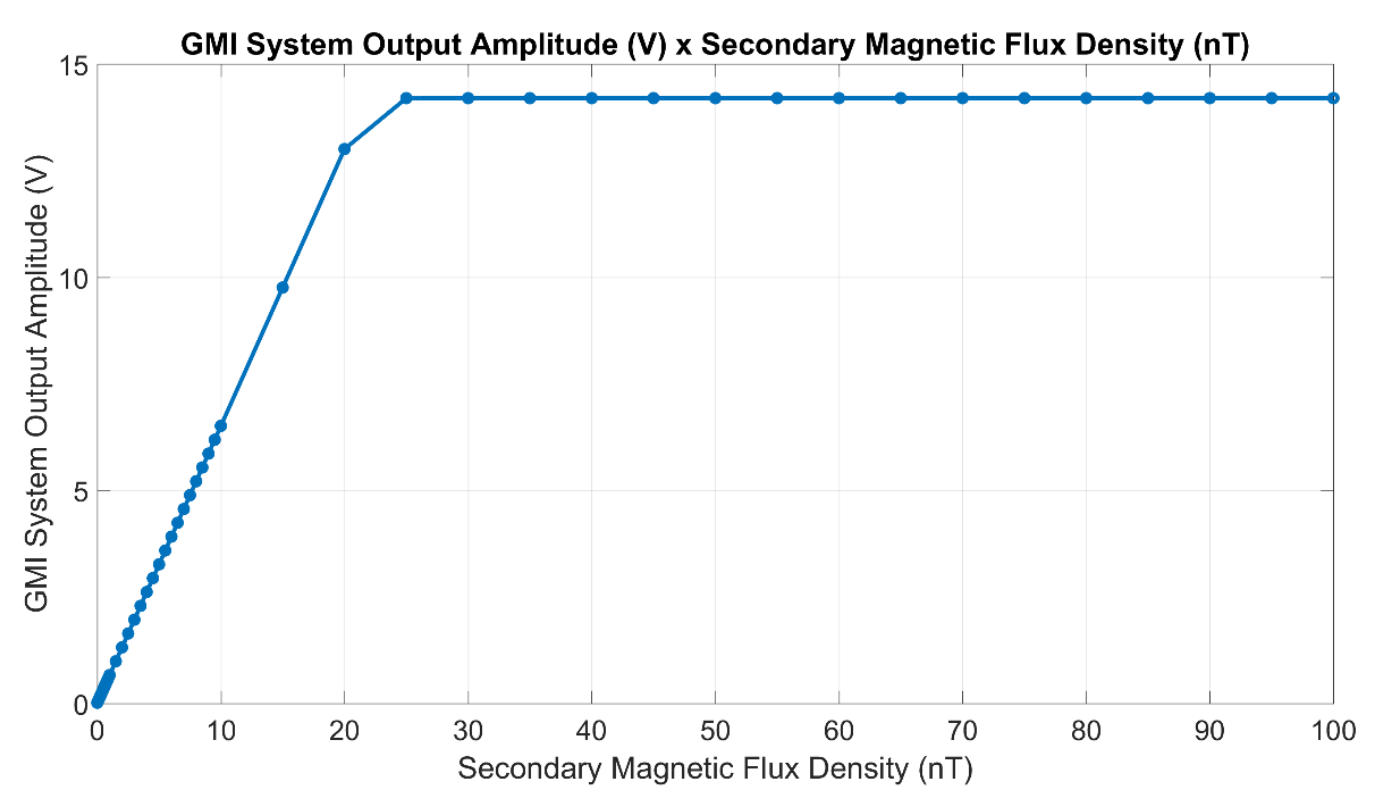

Figure 46 - GMI system output amplitude (V) for gain of $99.8 \mathrm{~V} / \mathrm{V}$ for secondary magnetic flux densities varying from 0 to $100 \mathrm{nT}$. 


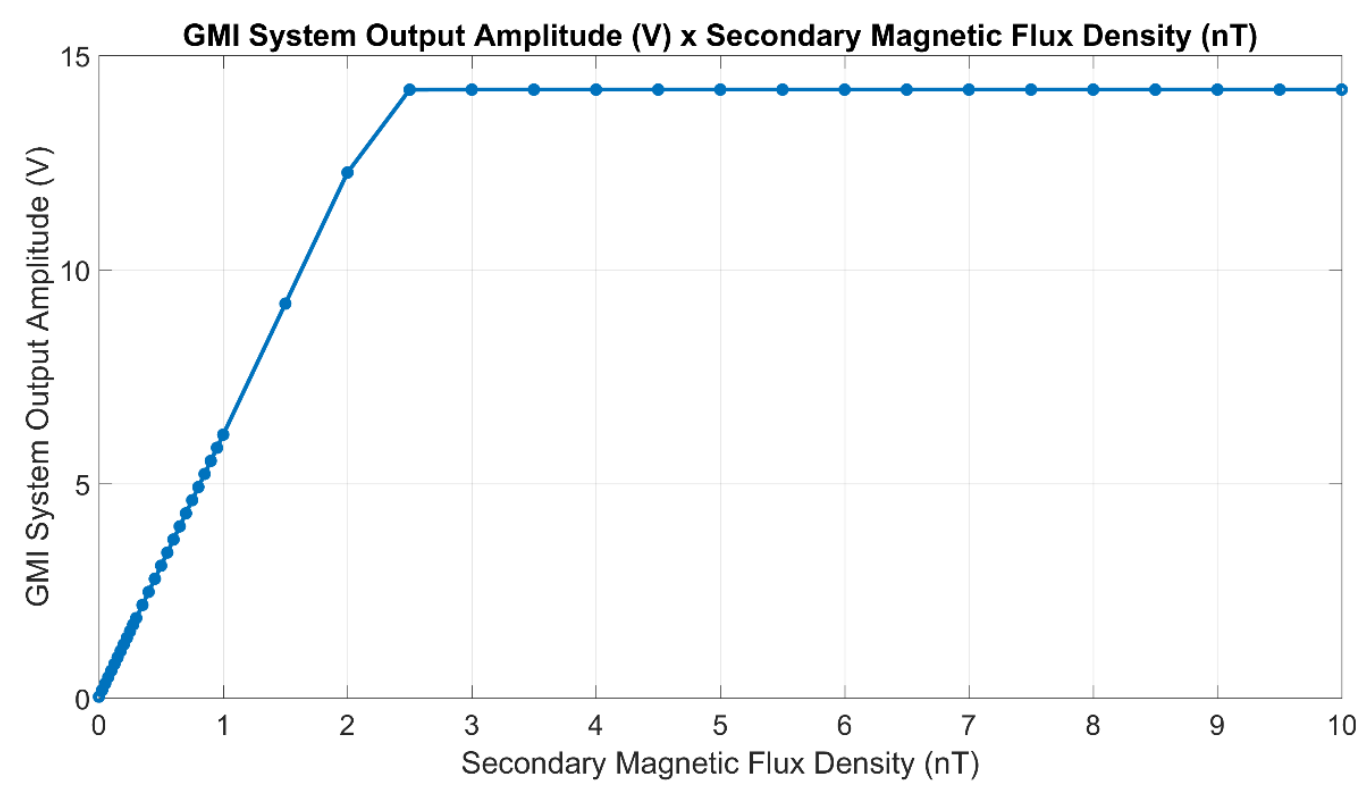

Figure 47 - GMI system output amplitude (V) for gain of $989 \mathrm{~V} / \mathrm{V}$ for secondary magnetic flux densities varying from 0 to $10 \mathrm{nT}$.

These results are consistent with the specified gains and the previously estimated maximum secondary magnetic flux density before the instrumentation amplifier output saturation. The ability to change the output gain allows for different detection ranges, always maintaining the desired signal at sufficiently readable levels above $100 \mathrm{mV}$ and increasing the signal-to-noise ratio (SNR).

Fig. 48 shows a logarithmic plot with all the gains used in the previous simulations and comparing to the experimentally characterized GMI sensitivity curve and the theoretical linear range of the sensor: 


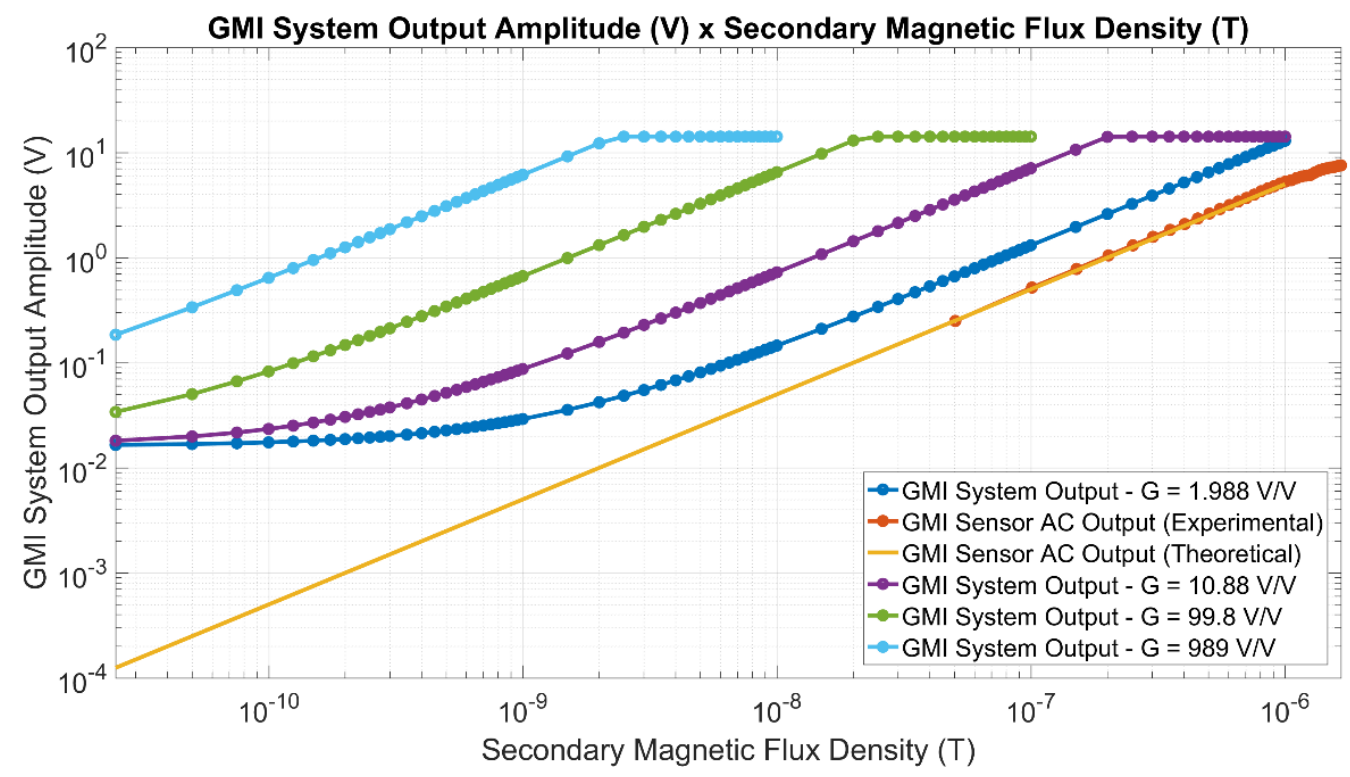

Figure 48 - Logarithmic plot for the GMI system output amplitude (V) versus secondary magnetic flux density including the four simulated gains, the experimental GMI characterization and the GMI theoretical linear range output.

This view facilitates a couple of key observations. The first is the presence of an amplitude offset ranging from $20 \mathrm{mV}$ to $200 \mathrm{mV}$ in the system output curves, mainly due to the CMRR. These offsets appear as a deviation from linearity in the logarithmic plot, but can in fact be removed as systematic errors without disturbing the actual sensitivity.

The second key observation is the determination of optimal measurement ranges, given in Table 6 . 
Table 6 - Optimal measurement ranges for each simulated differential gains in the GMI location system.

\begin{tabular}{|c|c|c|c|}
\hline $\begin{array}{c}\text { Gain } \\
\text { Resistor } \boldsymbol{R}_{\boldsymbol{G}} \\
(\Omega)\end{array}$ & $\begin{array}{c}\text { Differential } \\
\text { Gain } \boldsymbol{G}_{\boldsymbol{d i f}}(\mathrm{V} / \mathrm{V})\end{array}$ & $\begin{array}{c}\text { Lower Secondary } \\
\text { Magnetic Flux } \\
\text { Density Limit }\end{array}$ & $\begin{array}{c}\text { Upper Secondary } \\
\text { Magnetic Flux } \\
\text { Density Limit }\end{array}$ \\
\hline 50000 & 1.988 & $200 \mathrm{nT}$ & $>1 \mu \mathrm{T}$ \\
\hline 5000 & 10.88 & $20 \mathrm{nT}$ & $200 \mathrm{nT}$ \\
\hline 500 & 99.8 & $2 \mathrm{nT}$ & $20 \mathrm{nT}$ \\
\hline 50 & 989 & $25 \mathrm{pT}$ & $2 \mathrm{nT}$ \\
\hline
\end{tabular}

After removing the offset amplitude from the values, the point-by-point gain is given in Fig. 49.

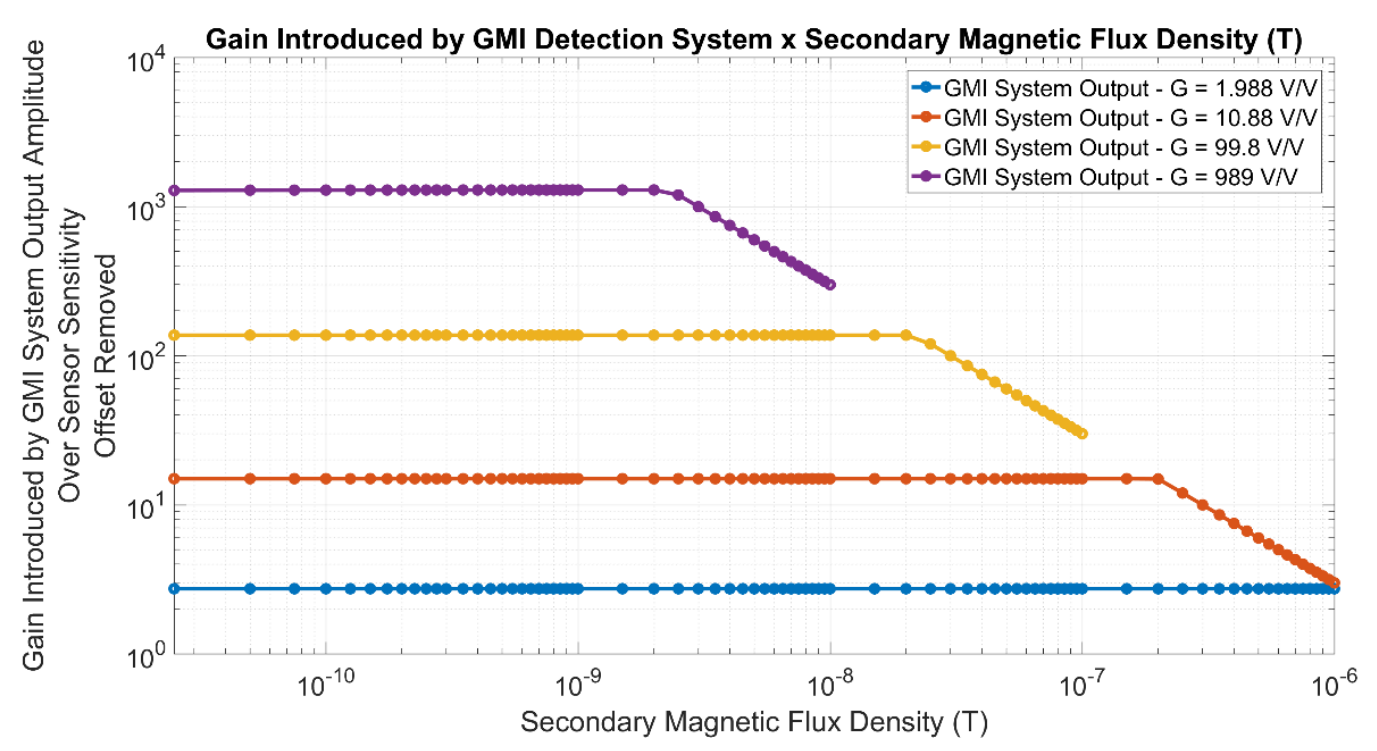

Figure 49 - Logarithmic plot for the gain introduced by the GMI detection system (V/V) versus secondary magnetic flux density for the four simulated gains. The amplitude offsets were removed.

Here the effective gains are confirmed to be within the expected values, which includes the small filter gain of around $1.488 \mathrm{~V} / \mathrm{V}$. Table 7 sums up the gain results. 
Table 7 - Comparison between theoretical and effective output gain $(\mathrm{V} / \mathrm{V})$ considering the simulation results.

\begin{tabular}{|c|c|c|c|c|}
\hline $\begin{array}{c}\text { Gain } \\
\text { Resistor } \boldsymbol{R}_{\boldsymbol{G}} \\
(\Omega)\end{array}$ & $\begin{array}{c}\text { INA129 } \\
\text { Differential } \\
\text { Gain } \boldsymbol{G}_{\text {dif }} \text { (V/V) }\end{array}$ & $\begin{array}{c}\text { Expected } \\
\text { Output Gain } \\
\text { (V/V) }\end{array}$ & $\begin{array}{c}\text { Effective } \\
\text { Output Gain } \\
\text { (V/V) }\end{array}$ & $\begin{array}{c}\text { Relative } \\
\text { Error (\%) }\end{array}$ \\
\hline 50000 & 1.988 & 2.96 & 2.73 & 7.68 \\
\hline 5000 & 10.88 & 16.19 & 14.95 & 7.66 \\
\hline 500 & 99.8 & 148.50 & 137 & 7.75 \\
\hline 50 & 989 & 1471.63 & 1290 & 12.3 \\
\hline
\end{tabular}

The relative error between theoretical values and the effective gain is usually under $8 \%$, except for the higher gain above $1000 \mathrm{~V} / \mathrm{V}$. This is expected with such elevated gains and should not heavily impact the system overall performance.

\subsubsection{6. \\ Secondary Magnetic Field Phase Effect on the System Output}

The mathematical models for eddy currents introduced in Chapter 3 show the phase between the secondary and primary magnetic flux densities are expected to be between $-90^{\circ}$ and $-170^{\circ}$. These phase values can be used to estimate the foreign body radius. However, phase differences can affect the amplitude of the location system output. It is important to verify whether this could impact the system resolution.

Fig. 50 shows a simulation of the system output voltage when varying the phase of the secondary magnetic flux density at the estimated resolution level of $25 \mathrm{pT}$ when using a gain of $989 \mathrm{~V} / \mathrm{V}$. 


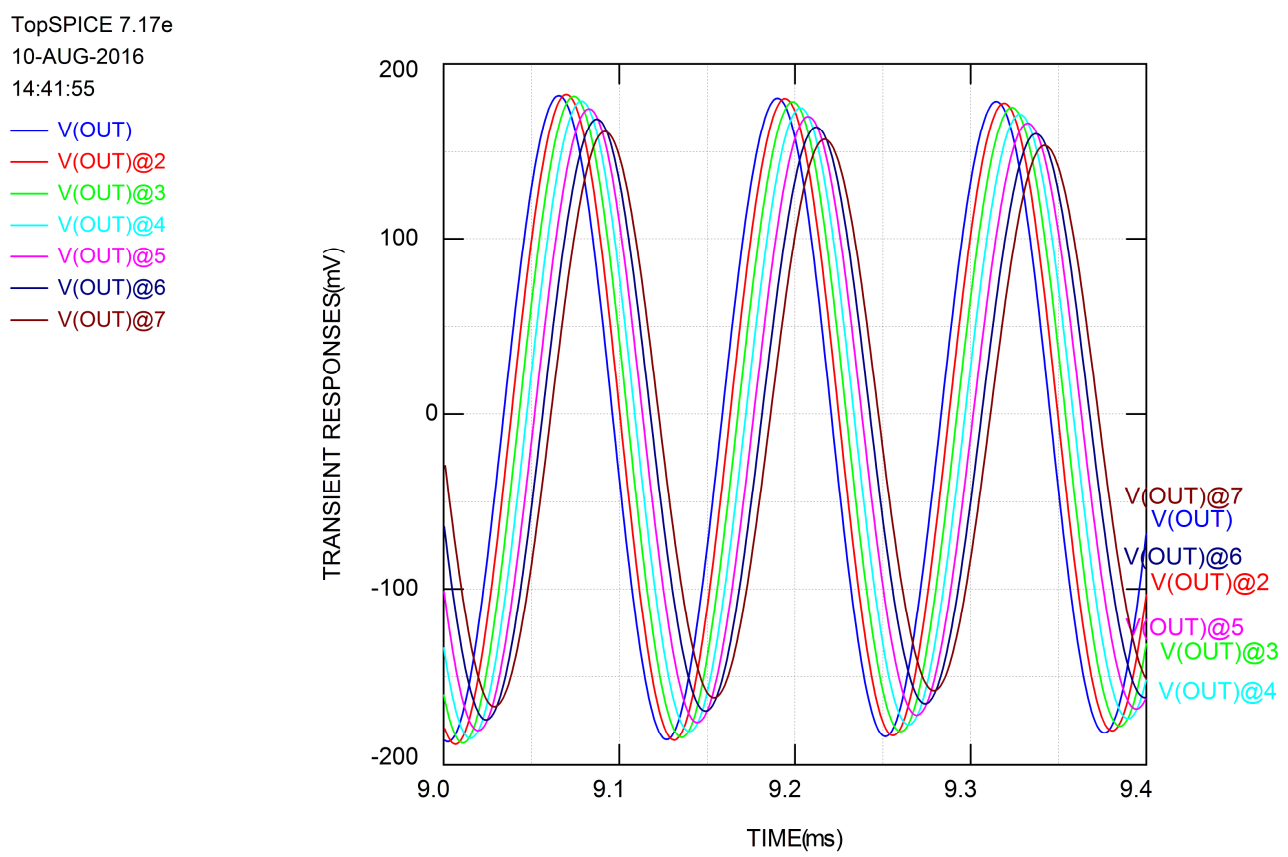

Figure 50 - GMI system output voltage under a secondary magnetic field of $25 \mathrm{pT}$ for $\phi_{\mathrm{sec}}$ phases between the primary and secondary magnetic fields ranging from $-90^{\circ}$ to $-180^{\circ}$ in $-15^{\circ}$ steps. The instrumentation amplifier gain is set at $989 \mathrm{~V} / \mathrm{V}$.

The variation due to the phase is noticeable, but does not seem to affect the resolution. Fig. 51 presents the response for different gains in the form of output amplitudes normalized by the value corresponding to a phase of $-90^{\circ}$.

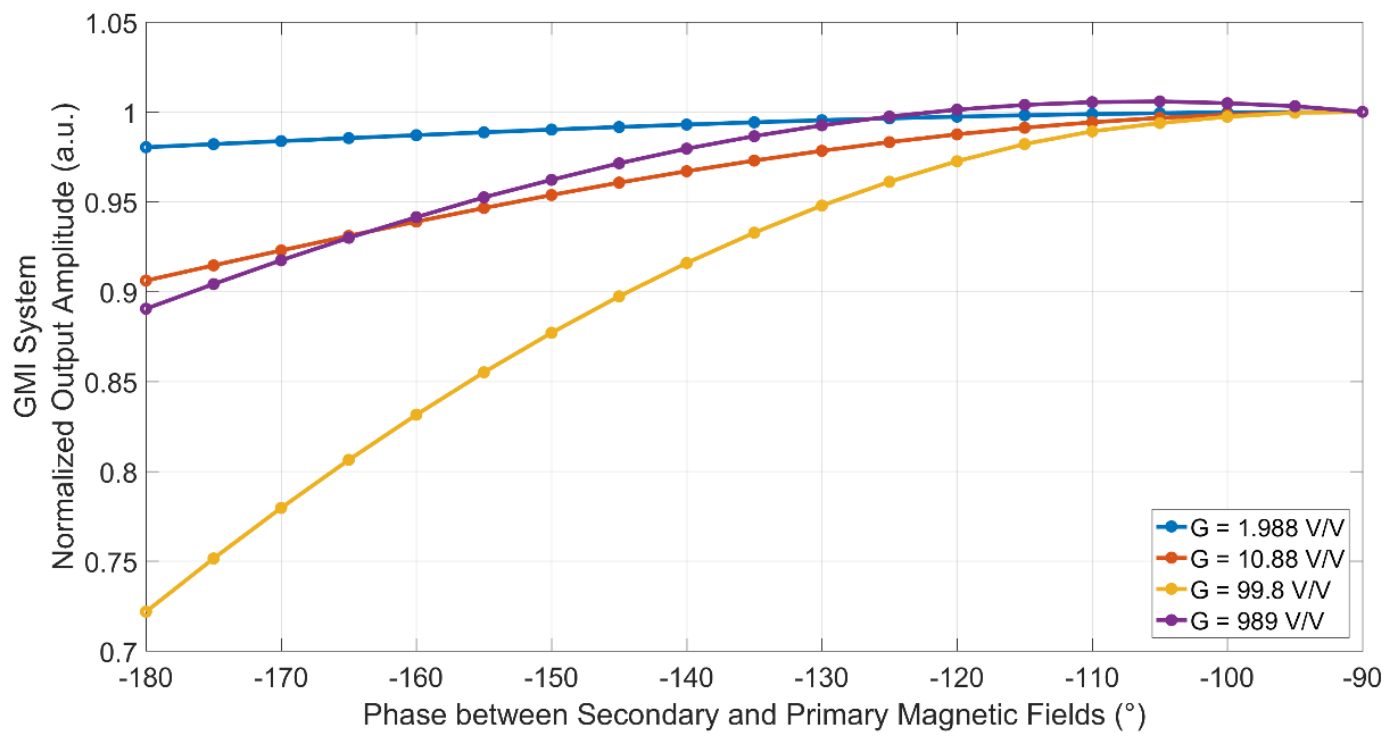

Figure 51 - GMI system normalized response to the secondary magnetic field phase for different gains, using the output amplitude at a phase of $\mathbf{- 9 0 ^ { \circ }}$ as reference. All values consider the $25 \mathrm{pT}$ resolution level. 
The output response for the resolution level of $25 \mathrm{pT}$ differs slightly for each gain, being most affected by the phase for a gain of $99.8 \mathrm{~V} / \mathrm{V}$. Considering the maximum phase to be around $-170^{\circ}$, the maximum output amplitude variation is under $25 \%$. Simulations for other secondary magnetic flux density values show the same general behavior. Therefore, the secondary phase variation has influence on the measurement uncertainty but does not affect the estimated system resolution. 
5

\section{Discussions, Conclusions and Future Works}

\section{1. \\ Discussions}

\subsection{1. Major Project Requirements for Biomedical Application}

The results obtained prove low cost magnetometers can be used as alternatives to SQUID sensors for the location of non-ferromagnetic metallic foreign bodies. The first pertinent analysis is to verify whether the proposed systems fulfill the major project requirements for biomedical devices [4].

\subsubsection{1. Innocuousness}

Innocuousness is a property missing from the currently available means of locating foreign bodies (x-ray photographs, radioscopy and computed tomography), which all use ionizing radiations. In the case of the proposed devices, it is mostly determined by the primary magnetic flux density emission and the safety levels established by ICNIRP guidelines. The currently published guidelines distinguish three frequency ranges for magnetic fields: Static (DC) [39], Low Frequency (1 Hz to $100 \mathrm{kHz}$ ) [40] and High Frequency (up to $300 \mathrm{GHz}$ ) [41]. All documents use root mean square magnetic flux density values for a more generic base of comparison.

The GMI location system is firmly on the Low Frequency range while the GMR system has a DC component and a $100 \mathrm{kHz}$ or higher AC component, requiring the consideration for both Low Frequency and High Frequency requirements. The SQUID system described in [3] is limited to a maximum frequency of $1 \mathrm{kHz}$, therefore it is also in the Low Frequency range.

The guidelines for Static fields state that occupational exposure of head and of trunk should not exceed $2 \mathrm{~T}$ while occupational exposure of limbs should not exceed $8 \mathrm{~T}$. For the general public, exposure of any part of the body should not 
exceed $400 \mathrm{mT}$. Anyone with implanted electronic medical devices and implants containing ferromagnetic material should have a more restrictive limit of $0.5 \mathrm{mT}$ [39].

The GMR locating system generates a static field of $4 \mathrm{mT}$, respecting the guidelines for both occupational and general public exposure. As expected, it is beyond the tolerated level for people with implanted devices, as they should avoid electromagnetic field emissions.

The High Frequency range guidelines consider the energy absorption from electromagnetic fields. For occupational exposure at a frequency $f$ in $\mathrm{MHz}$ between $0.0065 \mathrm{MHz}$ and $10 \mathrm{MHz}$, the magnetic flux density root mean square value should not exceed $2.0 \mu \mathrm{T} / f$. At the theoretical value of $100 \mathrm{kHz}$, this limit is $20 \mu \mathrm{T}$ and at the actual value of $123 \mathrm{kHz}$, it is $16.3 \mu \mathrm{T}$. For public exposure at frequencies between $3 \mathrm{kHz}$ and $150 \mathrm{kHz}$, the limit is fixed at $6.25 \mu \mathrm{T}$ [41].

The AC primary magnetic flux density for the GMR system at the edge of the solenoid is approximately $1.77 \mathrm{mT}$ in root mean square value. When considering the DC component, it is around $4.4 \mathrm{mT}$. These values surpass the exposure limits. Occupational exposure for medical staff can be minimized by attending a minimum distance requirement from the solenoid. The AC primary field must be attenuated about 11 times to meet the $16.3 \mu \mathrm{T}$ limit, which according to eq. (82) occurs at a distance of $9.6 \mathrm{~cm}$. Thus, maintaining the medical staff at least $9.6 \mathrm{~cm}$ away from the active solenoid is sufficient to respect the guidelines. Public exposure levels can be met at a $14.0 \mathrm{~cm}$ distance.

The Low Frequency guidelines consider the risks associated with nervous system transient responses. In the frequency range from $400 \mathrm{~Hz}$ to $3 \mathrm{kHz}$, the maximum magnetic flux density is $0.3 \mathrm{~T} / f$ for occupational exposure and $80 \mathrm{mT} / f$ for public exposure, where $f$ is the frequency in Hz. Therefore, at $1 \mathrm{kHz}$ the occupational exposure limit is $0.3 \mathrm{mT}$ and the public exposure limit is 80 $\mu \mathrm{T}$. For frequencies between $3 \mathrm{kHz}$ and $100 \mathrm{kHz}$, the rms magnetic flux density reference level for occupational exposure is $100 \mu \mathrm{T}$ while for public exposure it is $27 \mu \mathrm{T}[40]$.

For the GMR system, these reference levels are less restrictive than those proposed in the High Frequency guidelines, therefore it is recommended to use the latter. 
The solenoid considered for the SQUID system presented in [3] emits a primary magnetic flux density of $5.15 \mathrm{mT}$ in root mean square value. This high value is necessary due to the lower frequency bandwidth stipulated for this SQUID system, which leads to significantly smaller secondary magnetic flux densities. The proposed system would operate under a maximum frequency of $1 \mathrm{kHz}$. According to eq. (82), the occupational exposure reference level of $0.3 \mathrm{mT}$ is reached at a distance from the solenoid of $7.86 \mathrm{~cm}$ while the public exposure level of $80 \mu \mathrm{T}$ is met only at $14.1 \mathrm{~cm}$.

The primary magnetic flux density at the edge of the GMI system solenoid is $468 \mathrm{nT}$ in root mean square value. This is already below the reference levels for both occupational exposure $(100 \mu \mathrm{T})$ and public exposure $(27 \mu \mathrm{T})$. In fact, the primary magnetic flux density could be increased up to 57 times without surpassing general public exposure levels. This could be useful for improving the system performance by enabling higher secondary magnetic flux densities, however the GMI sensors would need to be removed from the primary magnetic field generator to avoid output saturation, requiring design changes. Table 8 summarizes these results.

Table 8 - Innocuousness analysis of the location systems using ICNIRP reference levels.

\begin{tabular}{|c|c|c|c|c|c|c|}
\hline System & Frequency & $\begin{array}{c}\text { Primary } \\
\text { Magnetic } \\
\text { Flux } \\
\text { Density } \\
\text { (rms) }\end{array}$ & $\begin{array}{c}\text { Occupational } \\
\text { Reference } \\
\text { Level }\end{array}$ & $\begin{array}{c}\text { General } \\
\text { Public } \\
\text { Reference } \\
\text { Level }\end{array}$ & $\begin{array}{l}\text { Safe Distance } \\
\text { Occupational }\end{array}$ & $\begin{array}{c}\text { Safe Distance } \\
\text { Public }\end{array}$ \\
\hline \multirow{2}{*}{ GMR } & $\mathrm{DC}$ & $4 \mathrm{mT}$ & $\begin{array}{l}2 \mathrm{~T} \\
{[39]}\end{array}$ & $\begin{array}{c}400 \mathrm{mT} \\
{[39]}\end{array}$ & Compliant & Compliant \\
\hline & $123 \mathrm{kHz}$ & $1.77 \mathrm{mT}$ & $\begin{array}{c}16.3 \mu \mathrm{T} \\
{[41]}\end{array}$ & $\begin{array}{c}6.25 \mu \mathrm{T} \\
{[41]}\end{array}$ & $9.6 \mathrm{~cm}$ & $14.0 \mathrm{~cm}$ \\
\hline GMI & $8 \mathrm{kHz}$ & $468 \mathrm{nT}$ & $\begin{array}{c}100 \mu \mathrm{T} \\
{[40]}\end{array}$ & $\begin{array}{r}27 \mu \mathrm{T} \\
{[40]}\end{array}$ & Compliant & Compliant \\
\hline SQUID & $1 \mathrm{kHz}$ & $5.15 \mathrm{mT}$ & $\begin{array}{c}0.3 \mathrm{mT} \\
{[40]}\end{array}$ & $\begin{array}{r}80 \mu \mathrm{T} \\
{[40]}\end{array}$ & $7.86 \mathrm{~cm}$ & $14.1 \mathrm{~cm}$ \\
\hline
\end{tabular}


Therefore, the GMI system is the most suitable for the requirement of innocuousness according to ICNIRP guidelines. Both the GMR and the SQUID systems have similar requirements for the distance from the active primary magnetic field generator in order to comply with the reference levels. This distance is reasonably small $(14.1 \mathrm{~cm}$ at most) but only the GMI system is inherently complying with the guidelines. Additionally, the use of magnetic field measurements instead of traditional imaging techniques is already a great advantage, heavily improving the innocuousness of metallic foreign body location techniques.

\subsubsection{2.}

\section{Non-invasiveness}

The use of magnetic field measurements for locating non-magnetic metallic foreign bodies is inherently a non-invasive procedure, thus any magnetometer complies with this requirement.

\subsubsection{3.}

\section{Low-cost}

Since prices can fluctuate due to a large number of variables, they can be difficult to estimate with accuracy. Nevertheless, it is a fact that SQUID magnetometers are complex devices depending on sophisticated manufacturing processes and maintenance. The need of cryogenic cooling in particular introduces the need for liquid helium recycling equipment as well as constant operational costs. A SQUID first-order gradiometer itself can cost over US\$100,000 and its continuous operation only increases this value. This high cost is prohibitive for clinical use and an important motivation for searching lower cost alternatives.

GMR sensors are very low-cost, since they have low manufacturing costs and are widely commercially available. The NVE sensor used or equivalents can be found under US $\$ 10$ apiece. This allows the GMR location system to be a true lowcost contender.

GMI sensors capable of measuring nanotesla magnetic flux density levels and below are still experimental, although already becoming commercially available, such as the Aichi prototype considered. GMI materials also have low manufacturing 
costs but usually require additional embedded electronics for properly optimizing the magnetometer performance due to the characteristics of the GMI effect, which slightly increases cost. The fact they are not widely commercially available also impacts the prices. Still, GMI prototypes are more than 100 times cheaper than SQUID magnetometers and do not introduce operational costs nor critical maintenance costs, being more than suitable for clinical use. Thus, the GMI location system can still be considered low-cost, although its price is currently much higher than the very low-cost GMR system.

\subsubsection{4. \\ Safety}

The general requirements for safety of medical electrical equipment are provided internationally by IEC and ISO standards, being incorporated in Brazil by Anvisa and INMETRO. There are currently no specific safety requirements for medical equipment dedicated to magnetic field measurements.

Aside from electrical safety, SQUID magnetometers have more crucial safety concerns in the handling of cryogenic cooling material. The primary magnetic field generators for both the SQUID and the GMR proposed location system have high currents requirements that lead to potential health risks, requiring more careful isolation protection for both patients and medical staff.

The GMI location system uses low currents and low voltages, therefore ordinary electrical safety requirements are sufficient. This makes it the contender that can more easily comply with safety requirements.

\subsubsection{5.}

\section{Portability}

This requirement facilitates the use of non-ferromagnetic metallic foreign body location techniques in clinical environments. For instance, a portable device would not require a dedicated room and has the potential of being easily used during surgical procedures.

SQUID magnetometers require liquid helium filled dewars and helium recycling devices, limiting them to very low portability. Usually, there is a dedicated room for SQUID measurements. 
Both GMI and GMR systems are very portable. The overall size depends mostly on the size of the solenoid and on the miniaturization of the electronic circuits.

\subsubsection{6.}

\section{Ease of use}

Elevated complexity can be detrimental for the widespread clinical use of a technique. The SQUID system, for instance, requires qualified personnel to handle the cryogenics and more critical maintenance requirements. On the other hand, both the GMI and the GMR systems are easily operated, requiring very little specific training. These devices could easily become one new tool in any surgical room.

\subsubsection{7. \\ Room temperature operation}

This is another requirement that avoids complexity of operation for clinical use. The SQUID, depending on superconducting effects, only operates at temperatures close to absolute zero, with a maximum of $77 \mathrm{~K}$ for high temperature SQUIDs. Both GMI and GMR comply with room temperature operation. Lower temperatures have benefits by reducing thermal effects on the sensitivity and noise levels but there is no need for extremely low temperatures.

\subsubsection{8.}

\section{Comparison}

In conclusion, the GMI system is the most compliant overall with the major project requirements for metrological reliability of medical electrical equipment. The SQUID is only favored for its higher sensitivity and lower noise level while GMR and GMI are more suitable for most of the other requirements. The only requirement in which the GMR system currently possesses an advantage over the GMI system is cost, but the difference is not prohibitive for the clinical environment, unlike the SQUID system.

The results from this analysis of the compliance with major project requirements by the proposed systems are summarized in Table 9. 
Table 9 - Analysis of the major biomedical device requirements for the considered magnetometers and their respective location systems.

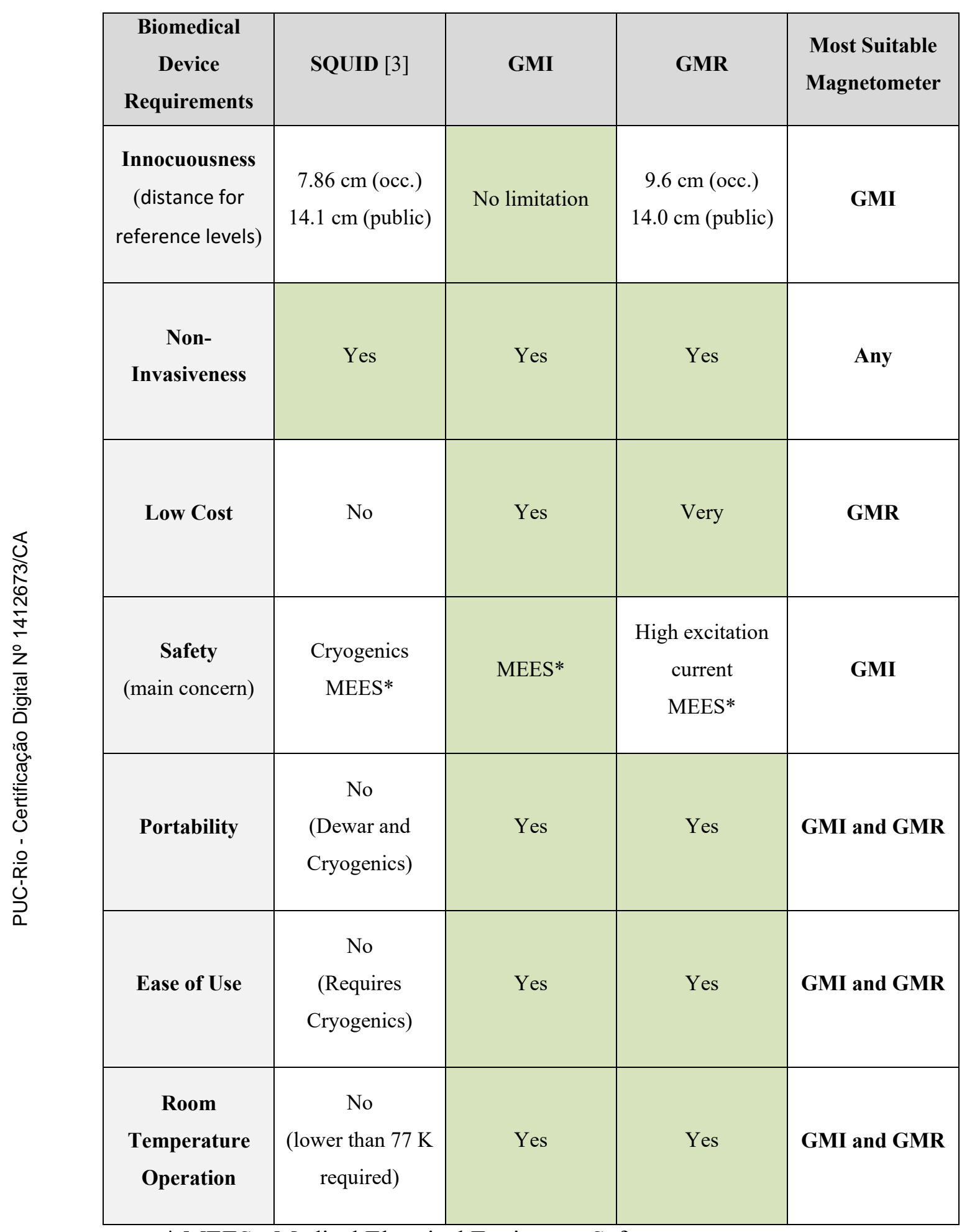

* MEES - Medical Electrical Equipment Safety 


\subsection{2. \\ Metrological Reliability}

Having established the requirements for biomedical devices, the remaining analysis is the metrological reliability, closely associated with the performance of the proposed systems in locating non-ferromagnetic metallic foreign objects.

\subsubsection{1. Metrological Traceability}

One of the most important elements for metrological reliability is the traceability of a quantity. This is established with the calibration of a device or technique by an accredited laboratory, allowing the measurements to be traced all the way up to the correspondent primary standard kept by the BIPM.

Currently, there is a single laboratory stated by the BIPM key comparison database [42] as qualified for calibrations related to AC magnetic flux densities under $100 \mathrm{nT}$. The United Kingdom National Physics Laboratory (NPL) performs calibrations for magnetometers and magnetic field generators in the range from $10 \mathrm{nT}$ to $22 \mathrm{mT}$, for frequencies between $10 \mathrm{~Hz}$ and $120 \mathrm{kHz}$. This is sufficient for metrological traceability of the primary magnetic field generators used in the proposed systems, however it does not cover the full range of secondary magnetic flux densities intended for detection.

Other institutions are capable of performing calibrations for the SQUID system's primary magnetic field generator, namely the Czech Metrology Institution (CMI) for the range between $0.5 \mu \mathrm{T}$ and $10 \mathrm{mT}$ at frequencies from $10 \mathrm{~Hz}$ to $50 \mathrm{kHz}$, the Netherlands VSL (Van Swinden Laboratory) for the range between $1 \mu \mathrm{T}$ and $20 \mathrm{mT}$ at frequencies from $10 \mathrm{~Hz}$ to $100 \mathrm{kHz}$ and the Polish Glowny Urzad Miar (GUM, or Central Office of Measures) for the range between $0.5 \mu \mathrm{T}$ and $11 \mathrm{mT}$ at frequencies from $10 \mathrm{~Hz}$ to $30 \mathrm{kHz}$. At this time in Brazil, INMETRO provides a restricted range of magnetic flux density standards from 1 $\mu \mathrm{T}$ to $700 \mu \mathrm{T}$ for the frequencies of $50 \mathrm{~Hz}$ and $60 \mathrm{~Hz}$ [16].

This limited magnitude and frequency range of available standards presents a great challenge for the measurement of ultra-low AC magnetic fields, such as those generated by the eddy currents in the proposed non-ferromagnetic foreign body location technique. Until there are appropriate international and national 
measurement standards available and institutions capable of providing metrological traceability for these magnetic fields, the measurement uncertainty and accuracy when dealing with these quantities will not be ensured. This also hinders the establishment of accuracy and measurement uncertainty requirements in international standards of this device which would be beneficial to the effective and widespread clinical use of the non-ferromagnetic metallic foreign body location device and technique.

\subsubsection{2.}

\section{High Sensitivity and Low Noise}

The most important attributes for the measurement of ultra-low magnetic fields are high sensitivity and the best possible resolution in order to detect magnetic flux densities caused by the eddy currents in the foreign bodies. SQUID magnetometers have an advantage in these criteria, with a noise density reaching $1 \mathrm{fT} / \sqrt{\mathrm{Hz}}$, more than 300000 times smaller than the considered alternatives. The Aichi GMI sensors come in second with $10 \mathrm{pT} / \sqrt{\mathrm{Hz}}$ and NVE GMR sensors are disadvantaged with $45.4 \mathrm{nT} / \sqrt{\mathrm{Hz}}$. These values are not definitive for these types of magnetometers, since there is room for improvement through technological advancements, especially for GMI sensors.

Under the operating conditions for each proposed location system, the estimated resolution would be $10 \mathrm{pT}$ for the SQUID system [3], $25 \mathrm{pT}$ for the GMI system and $2 \mu \mathrm{T}$ for the GMR system.

Actual sensitivity values usually depend on the signal amplification circuits. The most sensitive magnetometers, such as SQUIDs, have sensitivities as high as $10^{5} \mathrm{~V} / \mathrm{T}$ prior to any amplification [23]. In comparison, the Aichi GMI (which has an embedded amplification component) has $5 \cdot 10^{6} \mathrm{~V} / \mathrm{T}$ sensitivity while the NVE GMR can only reach a maximum of $156 \mathrm{~V} / \mathrm{T}$ without amplification. Having elevated sensitivity simplifies measurements, reduces component number and avoids introducing new noise sources in the circuits, as well as being associated with lower intrinsic noise levels.

Fig. 52 compares output amplitude versus secondary magnetic flux density for each sensor element and location system configuration in a logarithmic plot. The SQUID sensor characteristics were not specified in [3], therefore its output is 
estimated using typical magnetic flux density range and a sensitivity of $10^{5} \mathrm{~V} / \mathrm{T}$. GMR sensor output uses the datasheet specifications, labeled as theoretical. GMI sensor output is shown in both datasheet specifications (theoretical) and characterization results (experimental), the latter comprising a more limited range due to the difficulties in obtaining ultra-low magnetic fields from the utilized Helmholtz coil.

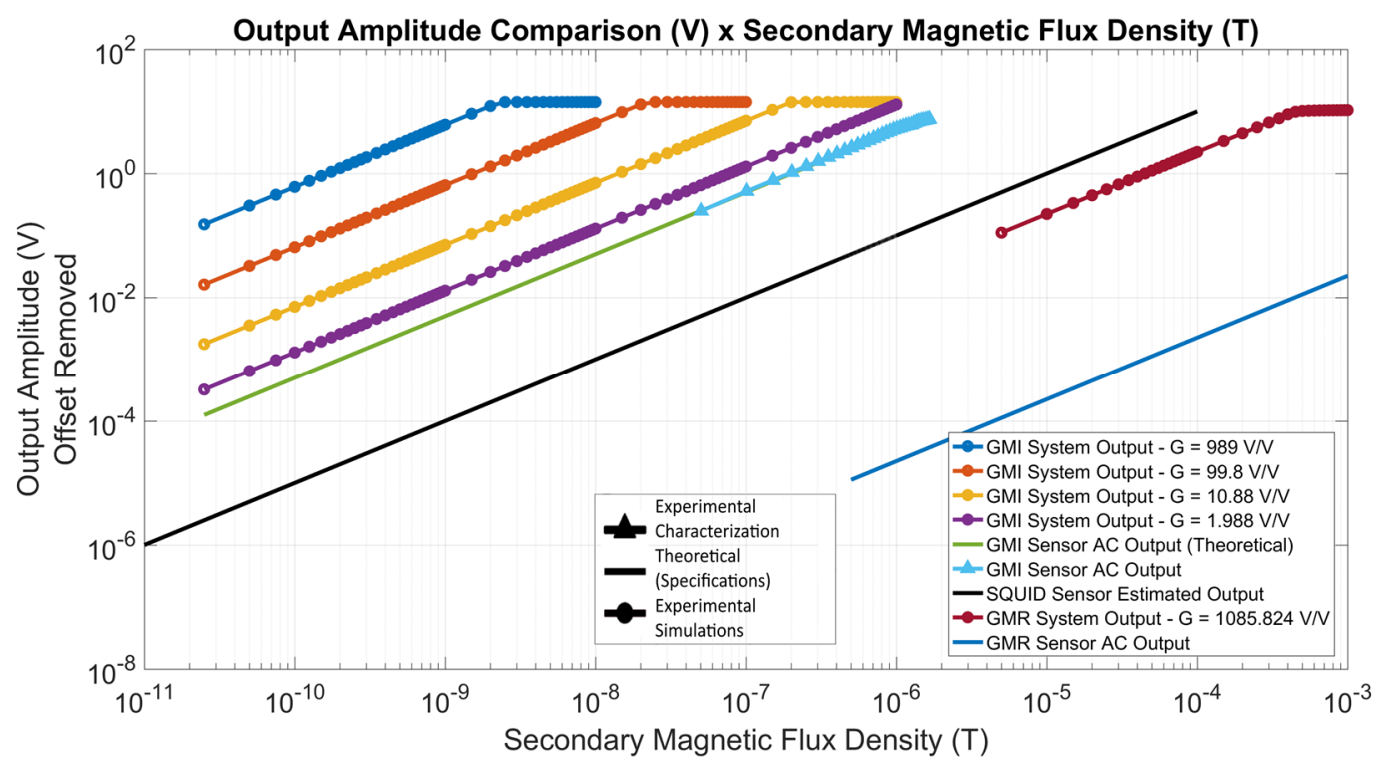

Figure 52 - Logarithmic plot comparison between sensor elements and location systems in output amplitude over secondary magnetic flux density.

The SQUID sensor operates with linearity on the largest magnetic flux density range, in part due to the lack of signal amplification, while also exhibiting very high sensitivity. Even at the estimated resolution of $10 \mathrm{pT}$ for the non-ferromagnetic foreign body location the output is at microvolt level.

The GMI sensor has an even higher sensitivity due to the embedded signal amplification, although its magnetic field range is more limited. The experimental characterization results obtained for magnetic flux densities over $40 \mathrm{nT}$ are compatible with the datasheet specifications. The location system with controllable gain permits considerable amplification, enabling an output amplitude around $150 \mathrm{mV}$ for a secondary magnetic flux density of $25 \mathrm{pT}$.

The GMR sensor has lower sensitivity and a more limited range. The location system amplification manages to almost match SQUID sensitivity while making the linear range even smaller and adding noise sources that limit resolution. 


\subsubsection{3.}

\section{Spatial Resolution}

As mentioned in Chapter 1, most magnetometers used as metal detectors sacrifice spatial resolution in order to achieve high sensitivity. However, the accurate location of small non-ferromagnetic metallic foreign bodies depends on improved spatial resolution. The magnetometers considered in this work have compact sensor elements, which leads to good spatial resolution.

SQUID sensor elements are the most compact, reaching nanometer dimensions, although the dewar for cryogenic cooling is much larger. GMR sensors are also compact, using semiconductor manufacturing technology. The bridge package of the NVE AA005-02 has micrometer dimensions. GMI sensors can be manufactured in several shapes such as amorphous wires, amorphous ribbons and multilayer films. Typically, they are presented in micrometer or millimeter dimensions. The three sensor elements satisfy the spatial resolution levels required to locate projectile foreign bodies.

\subsubsection{4. \\ Performance Comparison}

Initially, the performance of each system for locating non-ferromagnetic foreign bodies can be evaluated mainly by the minimum detectable diameter for varying distances and the output signal voltage levels. Higher voltage levels facilitate signal acquisition and post-processing, consequently improving the measurement uncertainty. These characteristics are closely related to the magnetometer characteristics. Posterior analyses would also include measurement uncertainty and accuracy, which would depend on the aforementioned metrological traceability. Fig. 53 presents the minimum detectable non-ferromagnetic metallic foreign body diameter versus height for the GMR and GMI systems described in this work and the SQUID system introduced in [3]. 


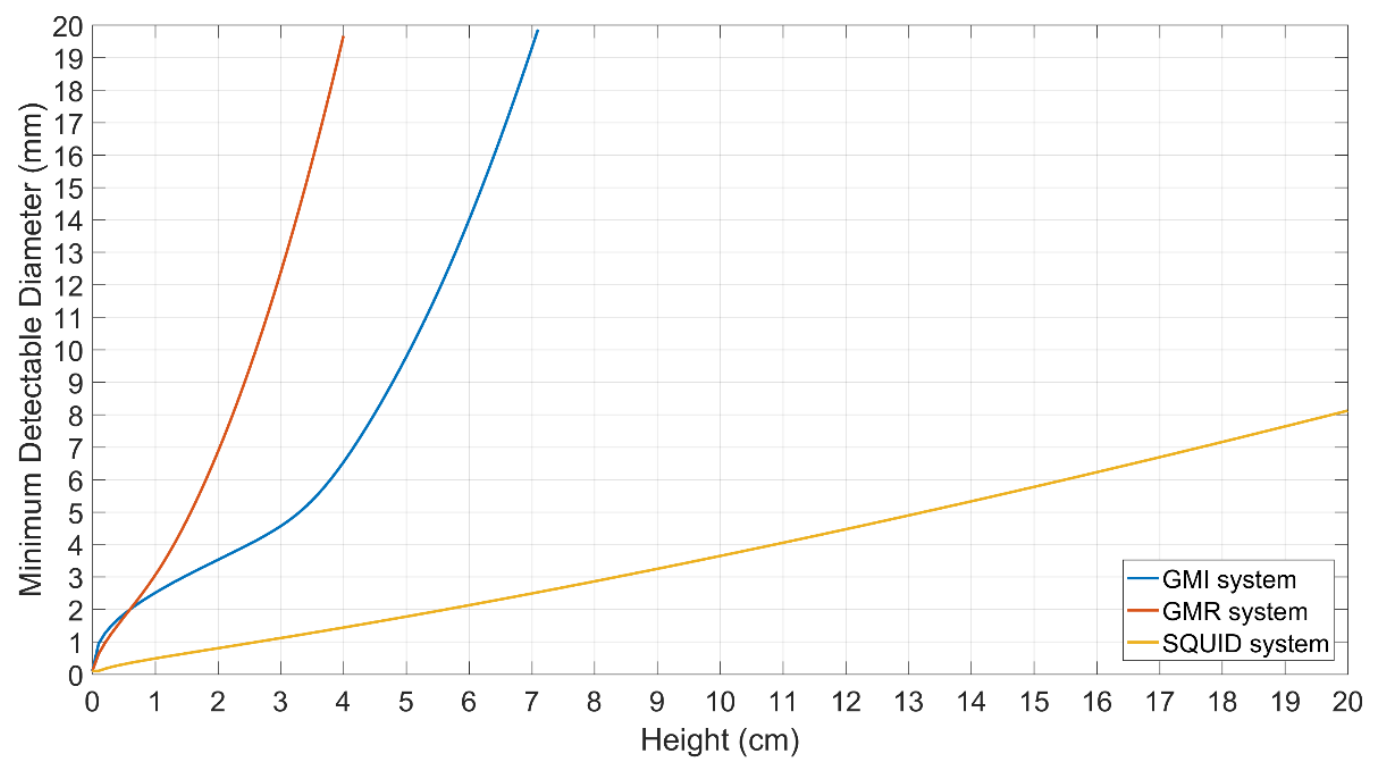

Figure 53 - Comparison of GMI, GMR and SQUID performance regarding minimum detectable diameter over height using the estimated resolutions for each detection system.

The SQUID system has a superior performance, as expected due to its current status as the most sensitive magnetometer. It is the only system capable of locating non-ferromagnetic foreign bodies at heights over $7 \mathrm{~cm}$ and also maintaining much lower detection thresholds. The GMI system comes in second, comparing very favorably against the GMR system by having more than twice the height range and detection thresholds halved above $2 \mathrm{~cm}$.

Another interesting analysis is to compare these results with new simulations where the primary magnetic field generators for each system use the respective ICNIRP reference levels without requiring any safety distance from the solenoids for neither occupational nor general public exposure, fulfilling the innocuousness requirement. For both the SQUID and GMR systems this would simply require reducing the excitation currents on the solenoids while the GMI system could greatly increase the magnetic fields, but it would entail a new design to avoid sensor output saturation. Fig. 54 introduces these results. 


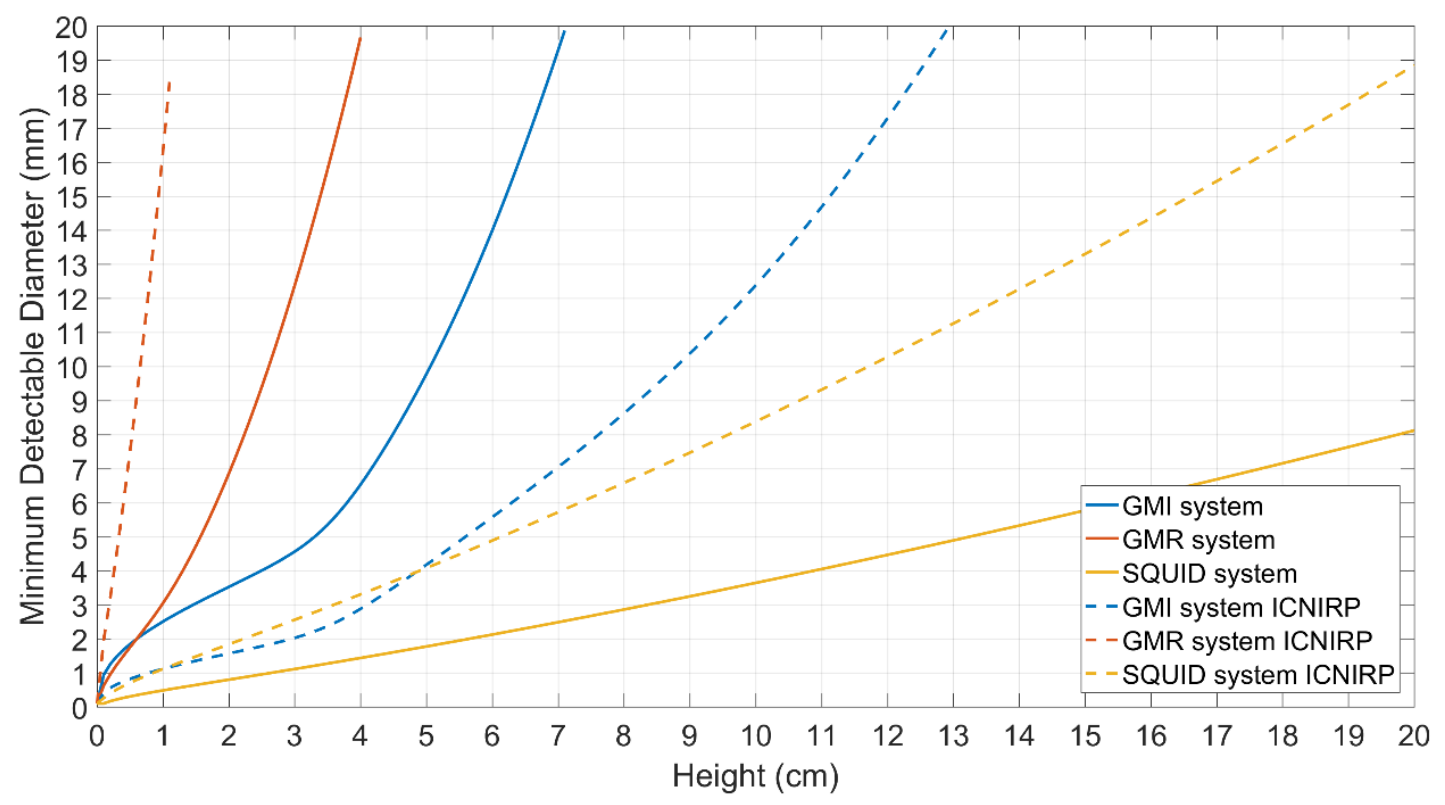

Figure 54 - Minimum detectable diameter over height for the proposed GMI, GMR and SQUID systems (solid lines) and adapted versions using the ICNIRP magnetic flux density reference levels, satisfying occupational and general public exposure at the corresponding frequencies (dashed lines).

Under these new ICNIRP compliant conditions, the GMI performance closely matches and occasionally surpasses the new SQUID performance for heights under $5 \mathrm{~cm}$. The GMI height range would expand to almost $13 \mathrm{~cm}$ for diameters over $20 \mathrm{~mm}$ and all detection thresholds are at least halved. SQUID performance maintains the highest height range while increasing minimum detectable diameters at least two times over the initial design. The GMR system is the most heavily and negatively impacted, losing almost any detection capabilities at heights over $1 \mathrm{~cm}$.

Table 10 summarizes the metrological reliability comparison between the proposed systems. 
Table 10 - Analysis of the major metrological reliability requirements for the considered magnetometers and their respective location systems.

\begin{tabular}{|c|c|c|c|c|}
\hline $\begin{array}{c}\text { Metrological } \\
\text { Reliability } \\
\text { Requirements }\end{array}$ & SQUID [3] & GMI & GMR & Most Suitable \\
\hline $\begin{array}{l}\text { Metrological } \\
\text { Traceability }\end{array}$ & $\begin{array}{l}\text { CMI, VSL, GUM } \\
\text { and NPL for } \\
\text { primary field }\end{array}$ & $\begin{array}{l}\text { NPL for primary } \\
\text { field }\end{array}$ & $\begin{array}{l}\text { NPL for primary } \\
\text { field }\end{array}$ & $\begin{array}{l}\text { Currently, only } \\
\text { primary field } \\
\text { generators in } \\
\text { few institutions }\end{array}$ \\
\hline $\begin{array}{c}\text { High Sensitivity } \\
\text { (sensor } \\
\text { sensitivity) }\end{array}$ & around $10^{5} \mathrm{~V} / \mathrm{T}$ & $\begin{array}{c}5 \cdot 10^{6} \mathrm{~V} / \mathrm{T} \\
\text { (with embedded } \\
\text { amplification) }\end{array}$ & $156 \mathrm{~V} / \mathrm{T}$ & GMI \\
\hline $\begin{array}{l}\text { Low Noise } \\
\text { Levels } \\
\text { (sensor noise } \\
\text { density) }\end{array}$ & $100 \mathrm{fT} / \sqrt{\mathrm{Hz}}$ & $10 \mathrm{pT} / \sqrt{\mathrm{Hz}}$ & $45.4 \mathrm{nT} / \sqrt{\mathrm{Hz}}$ & SQUID \\
\hline $\begin{array}{c}\text { Spatial } \\
\text { Resolution } \\
\text { (scale of sensor } \\
\text { dimensions) }\end{array}$ & $\begin{array}{c}\mathrm{cm} \\
(\text { down to } \mu \mathrm{m})\end{array}$ & $\begin{array}{c}\mathrm{mm} \\
\text { (down to } \mu \mathrm{m})\end{array}$ & $\mu \mathrm{m}$ & GMR \\
\hline $\begin{array}{c}\text { System } \\
\text { Resolution }\end{array}$ & $10 \mathrm{pT}$ & $25 \mathrm{pT}$ & $2 \mu \mathrm{T}$ & SQUID \\
\hline $\begin{array}{c}\text { Output } \\
\text { Amplitude at } \\
\text { System } \\
\text { Resolution }\end{array}$ & $\begin{array}{c}1 \mu \mathrm{V} \\
\text { (no external } \\
\text { gain) }\end{array}$ & $\begin{array}{c}152 \mathrm{mV} \\
\text { (with highest } \\
\text { gain) }\end{array}$ & $44.6 \mathrm{mV}$ & GMI \\
\hline $\begin{array}{l}\text { System Linear } \\
\text { Range } \\
\text { (magnetic flux } \\
\text { density) }\end{array}$ & $10 \mathrm{pT}$ to $100 \mu \mathrm{T}$ & $\begin{array}{c}25 \mathrm{pT} \text { to } 1 \mu \mathrm{T} \\
\text { (with lowest } \\
\text { gain) }\end{array}$ & $2 \mu \mathrm{T}$ to $400 \mu \mathrm{T}$ & SQUID \\
\hline $\begin{array}{l}\text { Foreign Body } \\
\text { Location } \\
\text { Performance }\end{array}$ & Highest & Intermediate & Lowest & SQUID \\
\hline
\end{tabular}


Overall, the SQUID system maintains the best performance and metrological reliability parameters. The GMI system proposed in this work stands in second place with very good characteristics. The SQUID system's failure to comply with many of the major project requirements for biomedical devices makes the GMI system the best option for clinical use [4]. The GMR system performance is much lower. In order to become an acceptable option, the use of a GMR sensor element with higher sensitivity would be required and it still would be difficult to match a GMI sensor.

\section{2. \\ Conclusions}

The simulation results presented in Chapter 4 and the discussions in Chapter 5 show the GMI system for the location of non-ferromagnetic metallic foreign bodies is capable of fulfilling its main objectives, mainly detecting secondary magnetic flux densities predicted to be generated by eddy currents in lead projectiles while also complying with the major biomedical device requirements that enable widespread clinical use. The GMR system can locate objects under a narrower range and comply with more biomedical requirements than the SQUID system, however its performance is currently not sufficient for a variety of real world cases.

GMI magnetometers dedicated to measuring nanotesla magnetic flux density levels are starting to become commercially available. This presents an opportunity for the application of low-cost magnetic field measurements in medicine, which can greatly benefit from the characteristics of non-invasiveness and innocuousness, as well as safety, portability and ease of use.

The particular application of locating non-magnetic metallic foreign bodies such as lead projectiles is a significant advancement towards reducing the often deadly consequences of being a victim of gunfire, an unfortunately commonplace situation. This technique can be readily applied to save countless lives and improve the quality of life of survivors.

It can also be applied to any other metallic foreign bodies, with even better performance results in cases of large metallic objects. This includes the use of this technique as a safety measure to verify if a patient does not have any metallic 
foreign body before undergoing an exam that relies on elevated magnetic fields, such as MRI. The presence of any metallic object makes the patient unfit for these exams, since it could lead to lethal injuries [43].

\section{3.}

\section{Future Works}

\subsection{1.}

\section{Optimizing the Location System Design}

The GMI system simulation results show the effectiveness of the proposed design, but it could be adapted further, after the pending first experimental tests of an electronic prototype. For instance, it is possible to implement an automatic control system that could use a digital potentiometer to change the instrumentation amplifier gain depending on the output signal, making a prototype even easier to use.

It is also shown in Fig. 54 that using a new primary magnetic field emitter with higher primary magnetic flux densities still within ICNIRP reference levels would greatly increase performance but would also require a new gradiometer design to avoid sensor output saturation.

Also of notice is the effect of phase differences between the signals in the final system output, as shown in Appendix B. Implementing a way to prevent phase mismatches between primary magnetic flux density signals is important to guarantee the reliability of measurements. On the other hand, accurately measuring the phase between secondary and primary signals would be useful for the location algorithms and could be performed by lock-in amplifiers, implemented either through hardware of software.

\subsection{2.}

\section{Signal Post-Processing and Location Algorithms}

The present work was limited in scope to the design of the electronic circuit components of the system for locating non-ferromagnetic foreign bodies. Before applying a system prototype to clinical tests, it would be necessary to study the best option for signal acquisition and post-processing, in particular the optimal location 
algorithm. The first step would be a laboratory testing of the location algorithm proposed in [3].

In more advanced studies, the magnetic field measurements could be converted into an imaging technique, projecting a magnetic field map similar to the technique for locating ferromagnetic steel needles [1]. It would be possible to use a position tracking device (an accelerometer, for example) to facilitate two-dimensional or three-dimensional mapping. This could in return lead back to new changes in the location system, such as implementing multiple channels of measurement. 


\section{Bibliography}

1 MONTEIRO, E. C. et al. Locating steel needles in the human body using a SQUID magnetometer. Physics in Medicine and Biology, v. 45, n. 8, p. 23892402, 2000.

2 BARBOSA, C. H. et al. Improvement of a Technique for Localization of Steel Needles in Humans Using a SQUID Magnetometer. IEEE TRANSACTIONS ON APPLIED SUPERCONDUCTIVITY, v. 11, n. 1, p. 677-680, 2001.

3 BARBOSA, C. H. Localization of firearm projectiles in the human body using a superconducting quantum interference device magnetometer: A theoretical study. Review of Scientific Instruments, v. 75, n. 6, p. 2098-2106, 2004.

4 COSTA MONTEIRO, E.; LEON, L. F. Metrological Reliability of Medical Devices. Journal of Physics: Conference Series. [S.1.]: IOP Publishing. 2015. p. 012032.

5 SMYTHE, W. R. Static and dynamic electricity. 3rd. ed. USA: Hemisphere Publ. Corp., 1989.

6 COSTA MONTEIRO, E. Biometrologia: confiabilidade nas biomedições e repercussões éticas. Metrologia e Instrumentação, n. 6, p. 6-12, 2007.

7 JCGM 200:2012. International vocabulary of metrology - Basic and general concepts and associated terms (VIM). 3rd Edition. ed. [S.1.]: [s.n.], 2012.

8 HENSON, A. Industrial and scientific metrology: road to a developed NMI. BIPM. [S.1.]. 2014.

9 BIPM. Available at: <http://www.bipm.org/en/about-us/>. Access on: Jan. 23rd, 2014.

10 OIML. Available at: <http://www.oiml.org/> Access on: Jan. 23rd, 2016.

11 WELCOME to the IEC - International Electrotechnical Commission. IEC International Electrotechnical Commission. Available at: $<$ http://www.iec.ch/>. Access on: Jan. 23rd, 2016.

12 ISO - International Organization for Standardization. ISO - International Organization for Standardization. Available at: $<$ http://www.iso.org/iso/home.html $>$. Access on: Jan. 23rd, 2016. 
13 WHO | World Health Organization. Available at: <http://www.who.int/en/>. Access on: Jan. 23rd, 2016.

14 ICNIRP. Available at: <http://www.icnirp.org/> Access on: Jan. 23rd, 2016.

15 Inmetro - Instituto Nacional de Metrologia, Qualidade e Tecnologia. Available at: <http://www.inmetro.gov.br/>. Access on: Jan. 23rd 2016.

16 INMETRO. INMETRO - Calibração - Divisão de Metrologia Elétrica. INMETRO. Available at: $<$ http://www.inmetro.gov.br/laboratorios/servicos/calibDiele.asp\#Lamce>. Access on: Aug. 08th, 2016.

17 INMETRO - Laboratórios de Metrologia Elétrica - Laboratório de Magnetismo e Campo Elétrico e Magnético - Lamce. Inmetro - Instituto Nacional de Metrologia, Qualidade e Tecnologia. Available at: $<$ http://www.inmetro.gov.br/metcientifica/eletrica/lamce.asp $>$. Access on: Jan. 23rd 2016.

18 Agência Nacional de Vigilância Sanitária - www.anvisa.gov.br. Available at: $<$ http://portal.anvisa.gov.br/wps/portal/anvisa/home $>$. Access on: Jan. 23rd 2016.

19 ABNT - Associação Brasileira de Normas Técnicas. Available at: $<$ http://www.abnt.org.br/>. Access on: Jan. 23rd 2016.

20 BUREAU INTERNATIONAL DES POIDS ET MESURES (BIPM). Le Système international d'unités (SI). 8. ed. Paris: STEDI MEDIA, v. 1, 2006.

21 ILMONIEMI, R. J. et al. Method for locating a small magnetic object in the human body. IEEE Transactions on Biomedical Engineering, July 1988. 561 $-564$.

22 LI, W.; WANG,. Magnetic Sensors for Navigation Applications: An Overview. The Journal of Navigation, v. 67, p. 263-275, Feb. 2014.

23 ROBBES, D. Highly sensitive magnetometers - a review. SENSORS AND ACTUATORS, A: PHYSICAL, v. 129, n. 1-2 Special Issue, p. 86-93, 2006.

24 RIPKA, P. Magnetic Sensors and Magnetometers. 1. ed. Norwood: Artech House Publishers, v. 1, 2001. ISBN ISBN:1-58053-057-5.

25 TIAN, Y.; YAN, S. Giant magnetoresistance: history, development and beyond. SCIENCE CHINA: Physics, Mechanics \& Astronomy, v. 56, n. 1, p. 2-14, 2013. 
26 SILVA, E. C. Magnetômetro GMI de Alta Sensibilidade para Medição de Campos Magnéticos Ultra-fracos. 2013. DSc Thesis Electrical Engineering Department, Pontifical Catholic University of Rio de Janeiro. Rio de Janeiro. 2013.

27 CARNEIRO, J. H. C. C. et al. System for automatic characterization of giant magneto-impedance samples. Proceedings of the XX IMEKO World Congress [Metrology for Green Growth]. Busan, Republic of Korea: [s.n.]. 2012. p. $1-5,9-14$.

28 COSTA SILVA, E. et al. Development of a fast and reliable system for the automatic characterization of Giant magnetoimpedance samples. ACTA IMEKO, v. 2, n. 1, p. 1-6, 2013.

29 COSTA SILVA, E. et al. An electronic approach to homogenize the impedance phase characteristics of heterogeneous GMI sensors. ACTA IMEKO, v. 1, n. 1, p. 70-76, 2012.

30 FORTALEZA, L. G. S. et al. Homogeneização das características de fase de amostras GMI para leitura gradiométrica. Proc. of the 10th International Congress on Electrical Metrology. Buenos Aires, Argentina: International Congress on Electrical Metrology - SEMETRO. 2013. p. 1-4.

31 SILVA, E. C. et al. High sensitivity giant magnetoimpedance (GMI) magnetic transducer: magnitude versus phase sensing. Measurement Science \& Technology (Print), v. 22, n. 3, p. 035204, 2011.

32 SILVA, E. C. et al. Point matching: A new electronic method for homogenizing the phase characteristics of giant magnetoimpedance. Review of Scientific Instruments, v. 85, n. 8, p. 084708 - 084708-5, 2014.

33 SILVA, E. C. et al. Homogenization of The Impedance Phase Characteristics of Giant Magnetoimpedance Sensors. Proceedings of the IX SEMETRO. Natal, Brasil: [s.n.]. 2011. p. 1-6.

34 HALL BARBOSA, C.; COSTA MONTEIRO, E.; POMPÉIA, F. Localization of Magnetic Foreign Bodies in Humans using Magnetic Field Sensors. Proceedings, XVII IMEKO World Congress. Dubrovnik: [s.n.]. 2003. p. 15101513.

35 SILVESTER, P. P.; OMERAGIC, D. Sensitivity Maps for Metal Detector Design. IEEE Transactions on Geoscience and Remote Sensing, 34, n. 3, May 1999. 788-792. 
36 FORTALEZA, L. G. E. S. et al. Detecting non-magnetic metallic foreign bodies by GMR sensors through the use of eddy currents. 8th Brazilian Congress on Metrology. Bento Gonçalves, RS: [s.n.]. 2015. p. 1-4. website:http://media.metrologia2015.org.br/media/uploads/trabalhos/SEMET RO_CBM_2015_Leonardo_rev_final.pdf.

37 FORTALEZA, L. G. E. S. et al. Electronic circuit for excitation of inductive loads with high currents. Electronics Letters, v. 55, n. 21, p. 1808 - 1809, out. 2015. ISSN ISSN:0013-5194. DOI:10.1049/el.2015.2587.

38 INDUCTIVELOAD. File:VCVS Filter Bandpass General.svg. Wikimedia Commons. Available

at: $<$ https://commons.wikimedia.org/wiki/File:VCVS_Filter_Bandpass_General.s $\operatorname{vg}>$.

39 INTERNATIONAL COMMISSION ON NON-IONIZING RADIATION PROTECTION. Guidelines on Limits of Exposure to Static Magnetic Fields.

Health Physics, v. 96, n. 4, p. 504-514, April 2009. ISSN doi: 10.1097/01.HP.0000343164.27920.4a. Available at: $<$ http://www.icnirp.org/cms/upload/publications/ICNIRPstatgdl.pdf>.

40 INTERNATIONAL COMMISSION ON NON-IONIZING RADIATION PROTECTION. Guidelines for Limiting Exposure to Time-Varying Electric and Magnetic Fields (1 Hz to 100 kHz). Health Physics, v. 99, n. 6, p. 818-836, December 2010. ISSN doi: 10.1097/HP.0b013e3181f06c86. Available at: $<$ http://www.icnirp.org/cms/upload/publications/ICNIRPLFgdl.pdf $>$.

41 INTERNATIONAL COMMISSION ON NON-IONIZING RADIATION PROTECTION. Guidelines for Limiting Exposure to Time-Varying Electric, Magnetic and Electromagnetic Fields (Up to 300 GHz). Health Physics, v. 74, n. $4, \quad$ p. 494-522, $1998 . \quad$ Available at: $<$ http://www.icnirp.org/cms/upload/publications/ICNIRPemfgdl.pdf $>$.

42 BIPM. The BIPM key comparison database. The BIPM key comparison database. Available at: <http://kcdb.bipm.org/>. Access on: Aug. 08th, 2016.

43 JAMES, C. ; KARACOZOFF, ; SHELLOCK, G. Undisclosed and undetected foreign bodies during MRI screening resulting in a potentially serious outcome. Magnetic Resonance Imaging, 31, 2013. 630-633. 


\section{Appendix A: Instrumentation Amplifier INA129}

The selected instrumentation amplifier for the experimental projects was the Texas Instruments INA129. It possesses several beneficial characteristics, such as wide bandwidth even for large gains, low offset voltage, wide supply range and high common-mode rejection ratio. These qualities enable the use of large gains and favor the analysis of very small signals.

The general equation for the output of an instrumentation amplifier is given by

$$
V_{O}=G_{d i f}\left(V_{i n+}-V_{i n-}\right)+G_{c m} \frac{\left(V_{i n+}+V_{i n-}\right)}{2},
$$

where $G_{d i f}$ is the differential gain, $G_{c m}$ is the common-mode gain and $V_{i n+}$ and $V_{i n-}$ are the inputs.

The differential gain $G_{\text {dif }}$ is given by

$$
G_{d i f}=1+\frac{49.4 \mathrm{k} \Omega}{R_{G}}
$$

where $R_{G}$ is the external gain resistance. It is important to consider that higher gains imply in smaller bandwidths due to Gain-Bandwidth Product (GBP) restrictions. The INA129 can operate with gains as high as $1000 \mathrm{~V} / \mathrm{V}$ for signals up to $10 \mathrm{kHz}$, but the bandwidth is smaller for signals of higher frequencies. Signals of $100 \mathrm{kHz}$ have maximum gain around $100 \mathrm{~V} / \mathrm{V}$.

The component's datasheet presents Common-Mode Rejection Ratio (CMMR) curves for varying frequencies and different differential gains $\left(G_{d i f}\right)$. The relation between CMRR (in $\mathrm{dB}$ ) and the amplifier gains (in $\mathrm{V} / \mathrm{V}$ ) is

$$
C M R R=20 \log _{10}\left(\frac{G_{d i f}}{\left|G_{c m}\right|}\right) .
$$

For frequencies around $8 \mathrm{kHz}$, the CMRR is between 50 and $110 \mathrm{~dB}$, with higher values for higher differential gains. This signifies that the differential gain is at least 316 times greater than the common-mode gain. For higher frequencies these values are smaller, for instance around $100 \mathrm{kHz}$ the CMRR ranges from 30 to $70 \mathrm{~dB}$. Higher CMRR reduces the influence of the common-mode voltage in the output. 
The tolerated signal of interest excursion due to output signal saturation levels can be estimated using eq. (109) and the information that for gains of 10 and $100 \mathrm{~V} / \mathrm{V}$ under a $\pm 15 \mathrm{~V}$ supply, the maximum output voltage is $13.75 \mathrm{~V}$. These values can be used to estimate the adequate gains for different secondary magnetic flux density ranges for each sensor type, ensuring both highest possible gain while avoiding output saturation. 


\section{Appendix B: Precision Full-Wave Rectifiers in the GMI System}

Initial studies were performed regarding the use of precision full-wave rectifiers at the band-pass filter outputs in order to reduce the consequences of phase mismatch between the signals at the instrumentation amplifier inputs. There are two main phases to consider in this project: the phase between the two signals pertaining to the primary magnetic field $\phi_{\text {pri }}$ and the phase between the secondary and the primary magnetic fields $\phi_{\text {sec. }}$ The signals would be in the form expressed in

$$
\left\{\begin{array}{c}
V_{1}=\mathrm{A} \cdot \sin \left(2 \cdot \pi \cdot f_{o}\right) \text { and } \\
V_{2}=\mathrm{A} \cdot \sin \left(2 \cdot \pi \cdot f_{o}+\boldsymbol{\phi}_{\text {pri }}\right)+\mathrm{B} \cdot \sin \left(2 \cdot \pi \cdot f_{o}+\boldsymbol{\phi}_{\text {sec }}\right)
\end{array}\right.
$$

where A stands for the voltage related to the primary magnetic flux density and B, to the secondary magnetic flux density.

The main source for the $\phi_{\text {pri }}$ mismatch is the existence of different phase delays introduced by the filters. Minute differences in component values are expected in practical situations, making this phase mismatch very likely.

When the AC input signals of an instrumentation amplifier have different phases, the output will not be zero even when amplitude and frequency are the same. This introduces a systematic error that can varies with the phase difference between

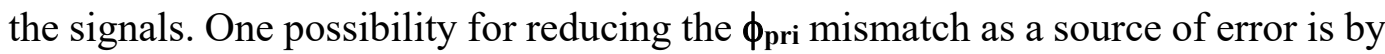
using rectifiers between the bandpass filters and the instrumentation amplifier.

The proposed precision full-wave rectifier design is shown in Fig. 55. The basic requirement for functioning is that resistors R2, R3, R4 and R5 have the same nominal values and small tolerance, typically under $1 \%$. It is recommended to use resistor arrays that possess very low tolerances. Using higher tolerance values increases the chance of instability in this topology. 


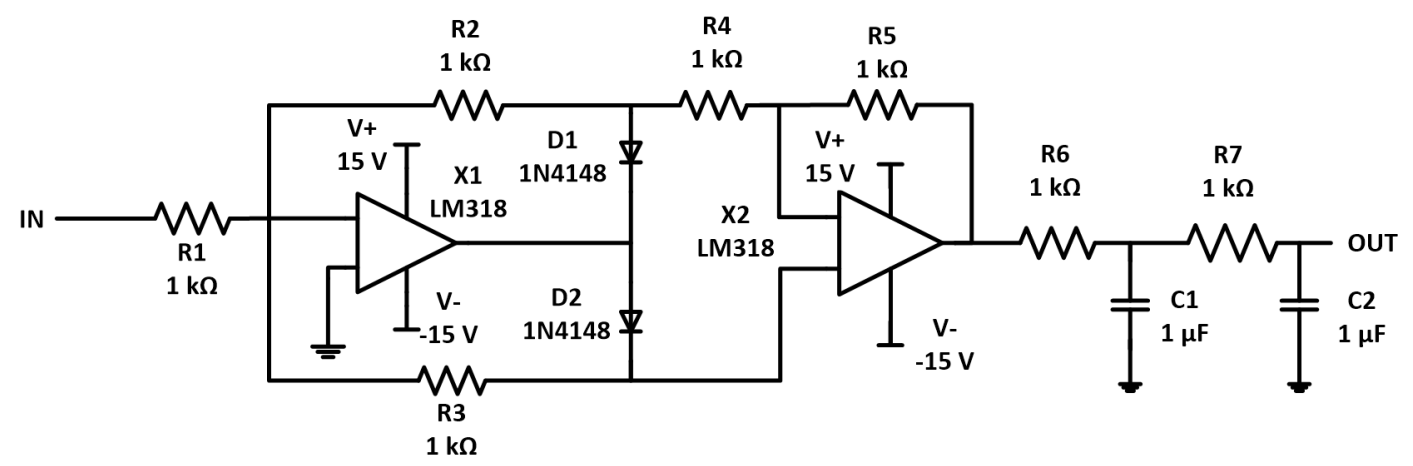

Figure 55 - Precision full-wave rectifier design intended to prevent phase mismatch between the instrumentation amplifier inputs. It requires that the resistors $R 2, R 3, R 4$ and $R 5$ have the same nominal value and very low tolerances while the input resistance $\mathrm{R} 1=R_{\text {in }}$ can be used for small gain adjustment.

This rectifier topology removes diode voltage drops from the output and allows the control of small gain adjustments, using eq. (113). This is useful in case the filters have small gain differences. The rectifier gain should be close to unity in order to maintain stability.

$$
V_{O}=\frac{R}{R_{\text {in }}} \cdot V_{\text {in }}
$$

Preliminary simulation results regarding $\phi_{\text {pri }}$ phase mismatches between the signals related to the primary magnetic fields are presented in Figs. 56 and 57. Fig. 56 shows the amplitudes of the instrumentation amplifier outputs under various $\phi_{\text {pri }}$ phases using a circuit without rectifiers while Fig. 57 presents directly the output of the instrumentation amplifier using the full-wave precision rectifiers. In both cases the amplifiers are configured with a gain of $99.8 \mathrm{~V} / \mathrm{V}$. 


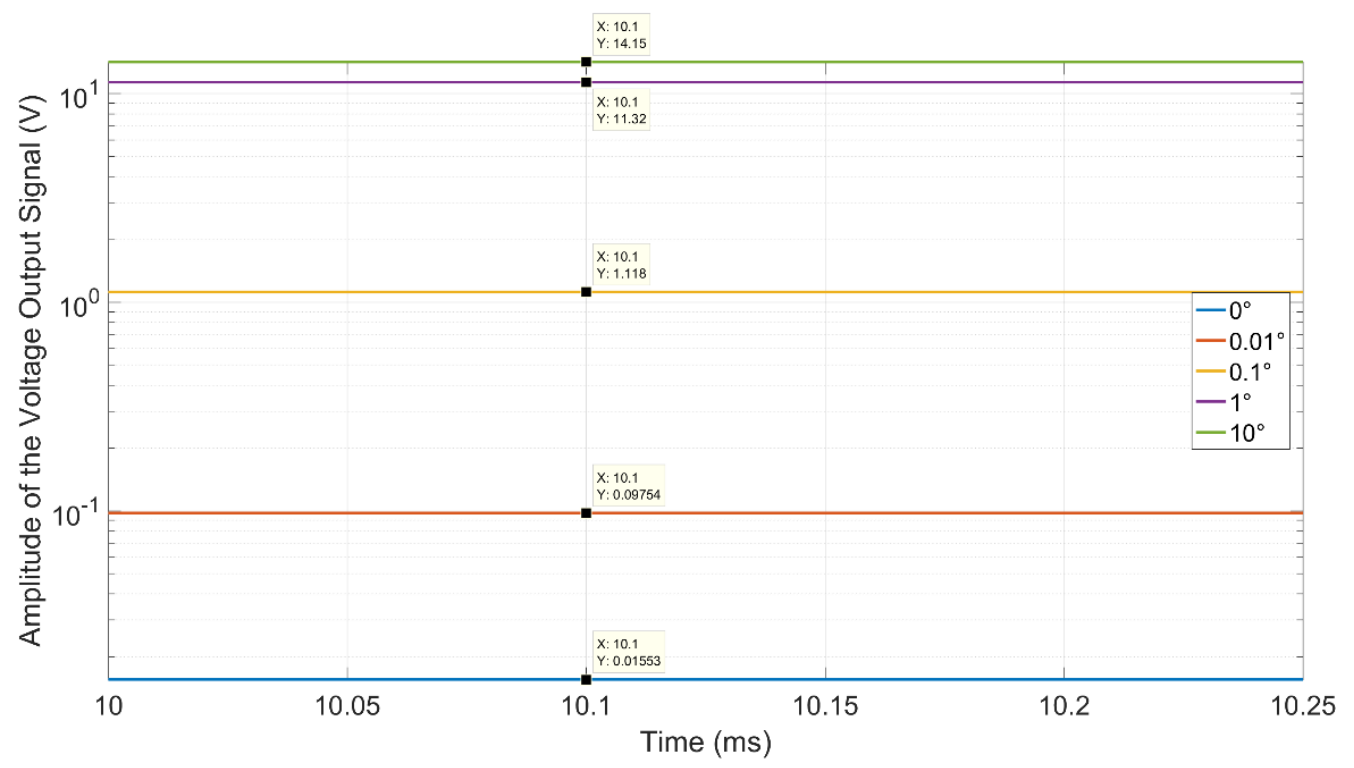

Figure 56 - Amplitudes of the instrumentation amplifier outputs (with 99.8 V/V gain) without any rectifier at various $\phi$ pri phase differences between the primary magnetic field signals, specifically: $0^{\circ}, 0.01^{\circ} 0.1^{\circ}, 1^{\circ}$ and $10^{\circ}$. The y-axis is in logarithmic scale.

Without the rectifiers, a very small $0.01^{\circ}$ phase mismatch already introduces a $97.54 \mathrm{mV}$ signal at the output and a $10^{\circ}$ phase mismatch leads to a saturated output reaching $14.15 \mathrm{~V}$. While very small phases around $0.01^{\circ}$ could be dealt with, its effect would still noticeably affect the readings. 


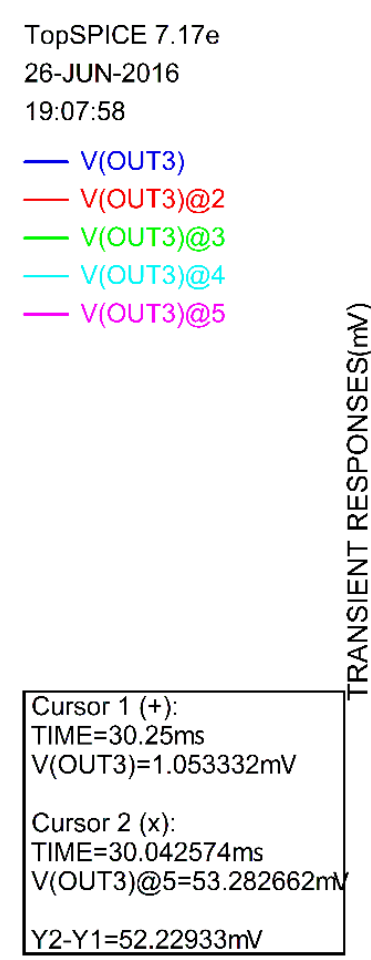

Figure 57 - Instrumentation amplifier outputs (with $99.8 \mathrm{~V} / \mathrm{V}$ gain) when using the precision rectifiers at various $\phi_{\text {pri }}$ phase differences between the primary magnetic field signals, specifically: $0^{\circ}, 0.01^{\circ}(@ 2), 0.1^{\circ}(@ 3), 1^{\circ}(@ 4)$ and10 $(@ 5)$.

When using the rectifier, the difference between the rectifier outputs reaches a maximum of $533 \mu \mathrm{V}$ with a $10^{\circ}$ mismatch, which leads to only $53.3 \mathrm{mV}$ at the instrumentation amplifier output. A more reasonable phase mismatch of $1^{\circ}$ has a rectifier output difference under $255 \mu \mathrm{V}$ and an amplifier output under $25 \mathrm{mV}$. However, in case the phase mismatch is high, the phase difference between the filters could be minimized by slightly varying the excitation frequency until an optimal operation point is located.

Values around a $0.1^{\circ}$ phase are under the expected sensor resolution which would represent $50 \mu \mathrm{V}$ at the rectifier output and $5 \mathrm{mV}$ at the instrumentation amplifier output. The phase mismatch related to the primary magnetic field would also be invariant under the same operation conditions, therefore the systematic error could be easily estimated and removed. These considerations lead to the conclusion that this circuit improves the performance in the case of a phase mismatch between the primary magnetic field signals but does not completely solves the issue. 
The $\phi_{\text {sec }}$ phase difference between the secondary magnetic field and the primary magnetic field is more critical to the circuit performance, since it is expected to be anywhere between $-90^{\circ}$ and $-170^{\circ}$. Figs. 58 and 59 show simulations using a secondary magnetic field signal with phase between $-90^{\circ}$ and $-180^{\circ}$ in $-15^{\circ}$ steps.
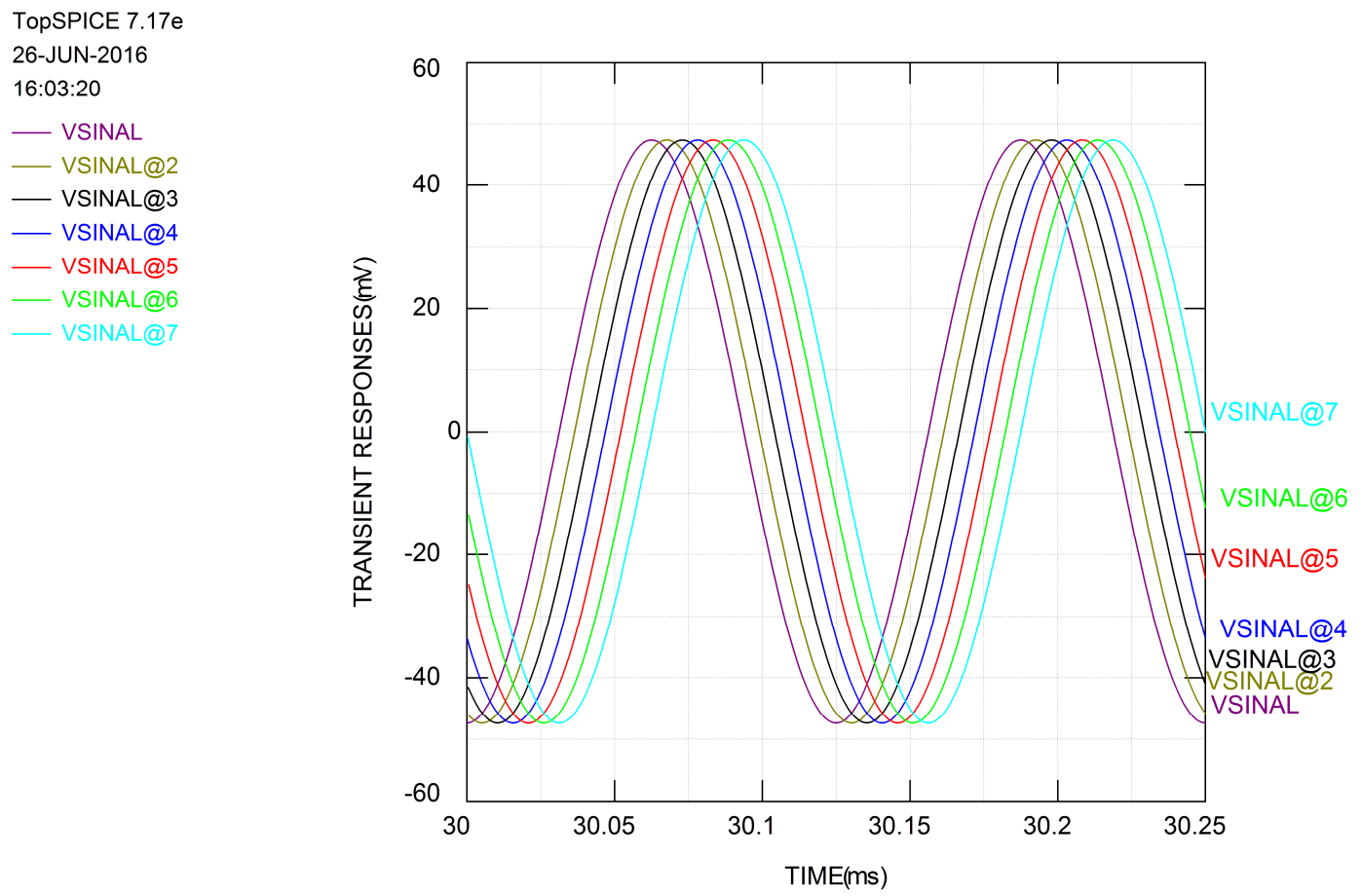

Figure 58 - Signal of interest under a secondary magnetic field of $10 \mathrm{nT}$ for $\phi_{\text {sec }}$ phases between the primary and secondary magnetic fields ranging from $-90^{\circ}$ to $-180^{\circ}$ in $-15^{\circ}$ steps. These signals were obtained in the simulations by mathematically subtracting the GMI sensor outputs. 
TopSPICE 7.17e
27-JUN-2016
17:37:49
- V(OUT3)
- V(OUT3)@2
- V(OUT3)@3
- V(OUT3)@4
- V(OUT3)@5
- V(OUT3)@6
- V(OUT3)@7

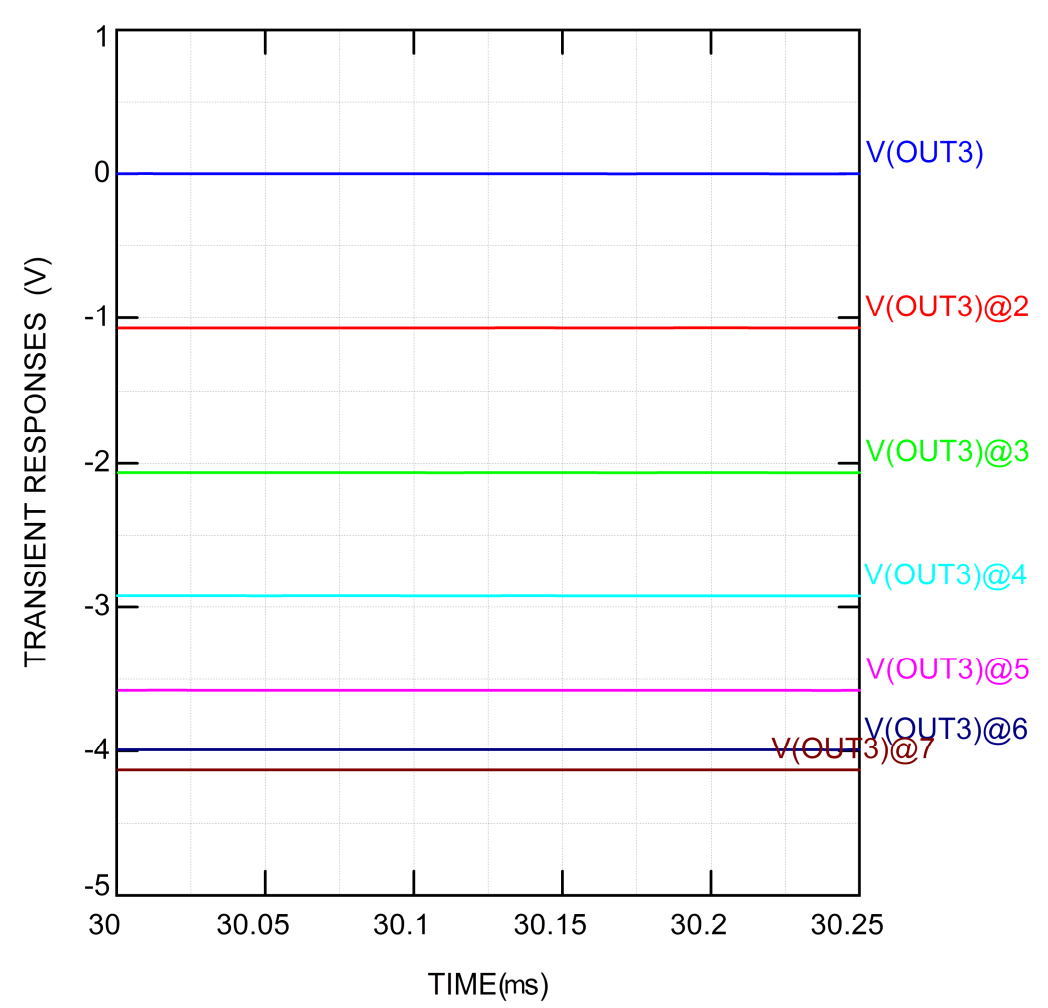

Figure 59 - Instrumentation amplifier outputs when using $99.8 \mathrm{~V} / \mathrm{V}$ gain and the precision rectifiers for a secondary magnetic flux density of $10 \mathrm{nT}$ with $\phi_{\text {sec }}$ phases between the primary and secondary magnetic fields ranging from $90^{\circ}$ to $-180^{\circ}$ in $-15^{\circ}$ steps.

These results indicate the $\phi_{\text {sec }}$ phase between the secondary and the primary magnetic fields can introduce voltage changes in the volt level at the instrumentation amplifier output, which greatly impacts the performance of the foreign body location system. It is also important to note the phasor nature of the quantity implies in having information in both amplitude and phase of the signal. The use of rectifiers scrambles these values in a way that the information related to the $\phi_{\text {sec }}$ phase is capable of completely overshadowing the amplitude, which is considered the most essential for foreign body location. Furthermore, this phase varies with the distance and the radius of the foreign body, making it very difficult to suppress its effects on the data.

The issues related to the $\phi_{\text {sec }}$ phase lead to the conclusion that the use of rectifiers before the instrumentation amplifier inputs are detrimental to the proposed foreign body location system. However, these studies are valuable in showing the impacts the $\phi_{\text {prim }}$ and $\phi_{\text {sec }}$ phases have on the final readings as well as the notion that the $\phi_{\text {sec }}$ 
also contains relevant information that needs to be preserved as separate from the amplitude before the gradiometric reading. 